\title{
Enantioselective Total Synthesis of (+)-Corymine and (-)-Deformylcorymine
}

\author{
Benxiang Zhang, ${ }^{\text {a }}$ Xiaoqing Wang, ${ }^{\text {a }}$ and Chaozhong $\mathrm{Li}^{*}$, a, b
}

${ }^{a}$ Key Laboratory of Organofluorine Chemistry, Center for Excellence in Molecular Synthesis, Shanghai Institute of Organic Chemistry, Chinese Academy of Sciences, 345 Lingling Road, Shanghai 200032, P. R. China, and ${ }^{\mathrm{b}}$ School of Materials and Chemical Engineering, Ningbo University of Technology, No. 201 Fenghua Road, Ningbo 315211, P. R. China. Email: clig@mail.sioc.ac.cn

\section{Table of Content}

1. Tables S1-S3.

2. Synthesis and characterizations of unexpected byproducts

3. Attempted synthesis of pseudoakuammigine and 17-hydroxypseudoakuammigine

4. Detailed procedures for the total synthesis of $(+)$-corymine and related alkaloids

5. ${ }^{1} \mathrm{H}$ and ${ }^{13} \mathrm{C}$ NMR spectra of all compounds. S27-S62

6. Comparison of spectra and data of natural and synthetic alkaloids

S63-S71

7. HPLC traces for measuring enantiomeric excesses 


\section{Tables $\mathrm{S} 1-\mathrm{S} 3$}

Table S1. Condition screening for enantioselective malonate addition of $\mathbf{1 1}$

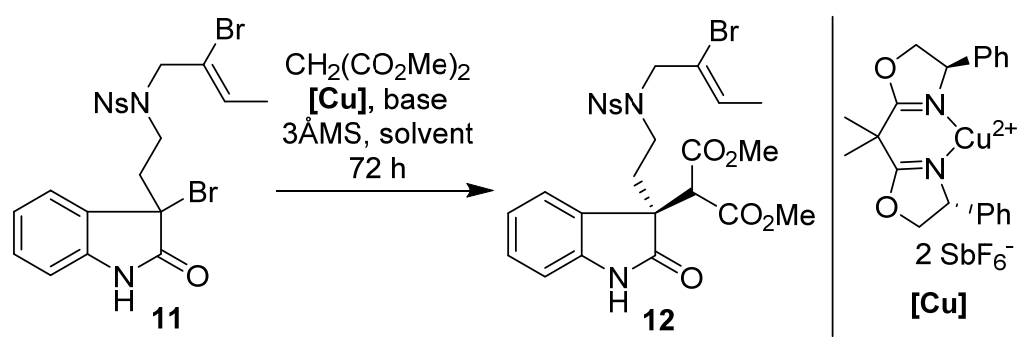

\begin{tabular}{|c|c|c|c|c|c|c|c|}
\hline entry $^{\mathrm{a}}$ & [Cu] (equiv) & $\mathrm{c}(\mathrm{mol} / \mathrm{L})$ & base & solvent & $\mathrm{T}\left({ }^{\mathrm{o}} \mathrm{C}\right)$ & yield $(\%)^{b}$ & ee $(\%)^{\mathrm{c}}$ \\
\hline 1 & 0.2 & 0.2 & DIPEA & $\mathrm{CH}_{2} \mathrm{Cl}_{2}$ & -20 & 45 & 73 \\
\hline 2 & 0.2 & 0.2 & DIPEA & $\mathrm{CH}_{2} \mathrm{Cl}_{2}$ & $\mathrm{rt}$ & 51 & 65 \\
\hline 3 & 0.2 & 0.2 & DIPEA & $\mathrm{CH}_{2} \mathrm{Cl}_{2}$ & -40 & 29 & 72 \\
\hline 4 & 0.2 & 0.1 & DIPEA & $\mathrm{CH}_{2} \mathrm{Cl}_{2}$ & -20 & 55 & 84 \\
\hline 5 & 0.2 & 0.05 & DIPEA & $\mathrm{CH}_{2} \mathrm{Cl}_{2}$ & -20 & 59 & 86 \\
\hline 6 & 0.3 & 0.05 & DIPEA & $\mathrm{CH}_{2} \mathrm{Cl}_{2}$ & -20 & 65 & 91 \\
\hline 7 & 0.3 & 0.05 & $\mathrm{Et}_{3} \mathrm{~N}$ & $\mathrm{CH}_{2} \mathrm{Cl}_{2}$ & -20 & 60 & 82 \\
\hline 8 & 0.3 & 0.05 & $\mathrm{Cy}_{2} \mathrm{NMe}$ & $\mathrm{CH}_{2} \mathrm{Cl}_{2}$ & -20 & 58 & 84 \\
\hline 9 & 0.3 & 0.05 & $\mathrm{~K}_{3} \mathrm{PO}_{4}$ & $\mathrm{CH}_{2} \mathrm{Cl}_{2}$ & -20 & - & - \\
\hline 10 & 0.3 & 0.05 & $\mathrm{CS}_{2} \mathrm{CO}_{3}$ & $\mathrm{CH}_{2} \mathrm{Cl}_{2}$ & -20 & - & - \\
\hline 11 & 0.3 & 0.05 & DIPEA & THF & -20 & 45 & 66 \\
\hline 12 & 0.3 & 0.05 & DIPEA & MTBE & -20 & - & - \\
\hline 13 & 0.3 & 0.05 & DIPEA & $\mathrm{CH}_{3} \mathrm{CN}$ & -20 & 26 & 40 \\
\hline 14 & 0.3 & 0.05 & DIPEA & DMF & -20 & - & - \\
\hline
\end{tabular}

${ }^{\mathrm{a}}$ Reaction conditions: $11(0.1 \mathrm{mmol}), \mathrm{CH}_{2}\left(\mathrm{CO}_{2} \mathrm{Me}\right)_{2}(0.3 \mathrm{mmol})$, base $(0.2 \mathrm{mmol}), 3 \AA \mathrm{MS}(32.3$ $\mathrm{mg}){ }^{\mathrm{b}}$ Isolated yield. ${ }^{\mathrm{c}}$ Measured by chiral HPLC. 
Table S2. Condition screening for Suzuki coupling of 13
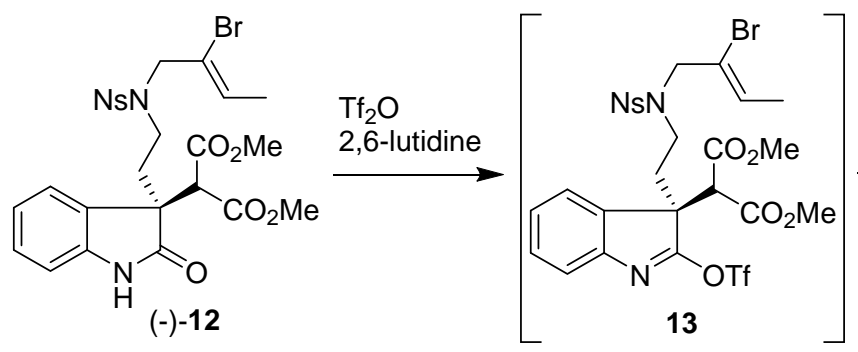

14, [Pd], ligand base, solvent
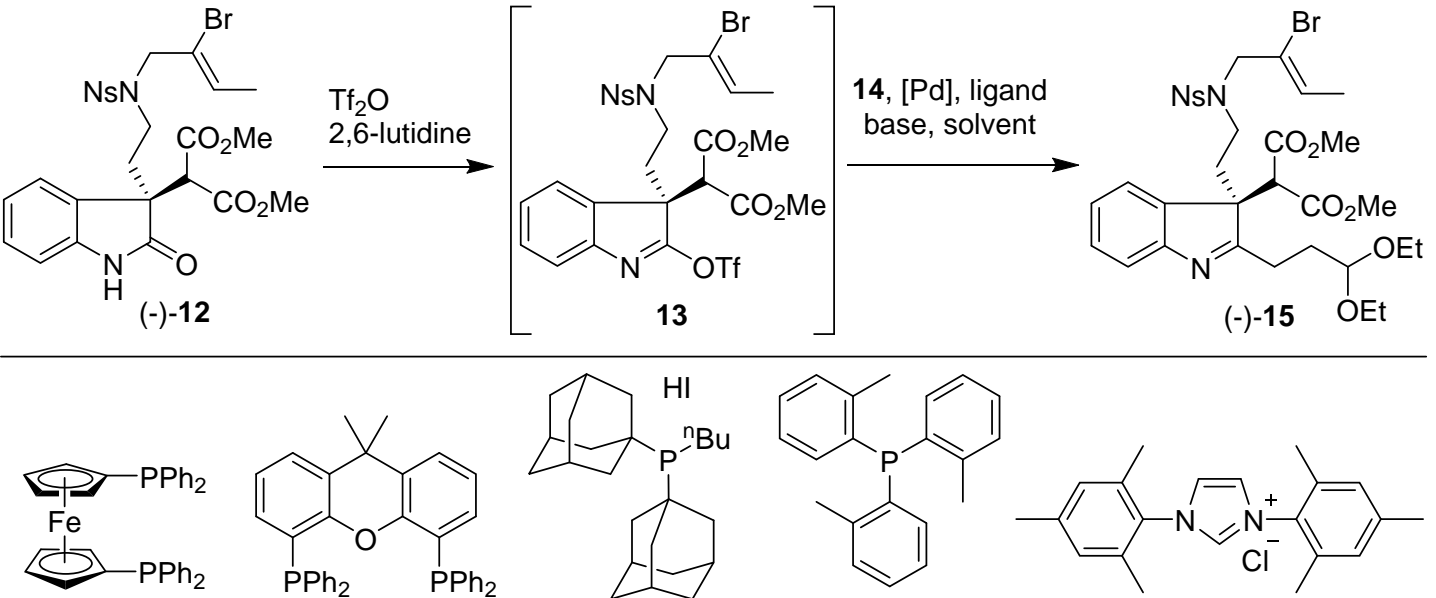

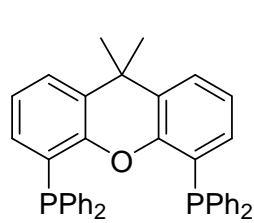

L2

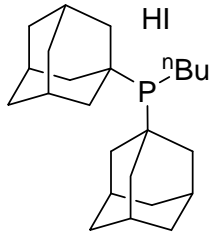

L3

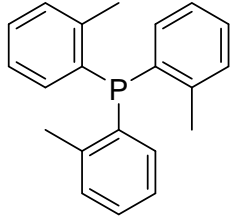

44

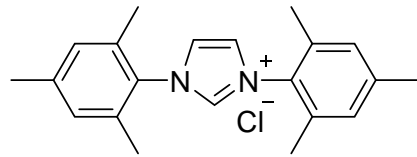

L5

\begin{tabular}{|c|c|c|c|c|c|}
\hline entry $^{a}$ & {$[\mathrm{Pd}]$} & ligand & base & solvent & yield $(\%)^{b}$ \\
\hline $1^{\mathrm{c}}$ & $\mathrm{PdCl}_{2}\left(\mathrm{PPh}_{3}\right)_{2}$ & / & ${ }^{t} \mathrm{BuOLi}$ & THF & 10 \\
\hline 2 & $\mathrm{PdCl}_{2}\left(\mathrm{PPh}_{3}\right)_{2}$ & / & $\mathrm{K}_{3} \mathrm{PO}_{4}$ & THF & 31 \\
\hline 3 & $\mathrm{PdCl}_{2}\left(\mathrm{PPh}_{3}\right)_{2}$ & / & $\mathrm{K}_{2} \mathrm{CO}_{3}$ & THF & 40 \\
\hline 4 & $\mathrm{PdCl}_{2}\left(\mathrm{PPh}_{3}\right)_{2}$ & / & $\mathrm{Et}_{3} \mathrm{~N}$ & THF & 15 \\
\hline 5 & $\mathrm{PdCl}_{2}\left(\mathrm{PPh}_{3}\right)_{2}$ & / & $\mathrm{K}_{2} \mathrm{CO}_{3}$ & DCE & 22 \\
\hline 6 & $\mathrm{PdCl}_{2}\left(\mathrm{PPh}_{3}\right)_{2}$ & / & $\mathrm{K}_{2} \mathrm{CO}_{3}$ & DMF & 0 \\
\hline 7 & $\mathrm{PdCl}_{2}\left(\mathrm{PPh}_{3}\right)_{2}$ & / & $\mathrm{K}_{2} \mathrm{CO}_{3}$ & dioxane & 20 \\
\hline 8 & $\mathrm{PdCl}_{2}\left(\mathrm{PPh}_{3}\right)_{2}$ & l & $\mathrm{K}_{2} \mathrm{CO}_{3}$ & toluene & 14 \\
\hline 9 & $\mathrm{PdCl}_{2}\left(\mathrm{PPh}_{3}\right)_{2}$ & $\mathbf{L 1}$ & $\mathrm{K}_{2} \mathrm{CO}_{3}$ & THF & 10 \\
\hline 10 & $\mathrm{PdCl}_{2}\left(\mathrm{PPh}_{3}\right)_{2}$ & L2 & $\mathrm{K}_{2} \mathrm{CO}_{3}$ & THF & 0 \\
\hline 11 & $\mathrm{PdCl}_{2}\left(\mathrm{PPh}_{3}\right)_{2}$ & L3 & $\mathrm{K}_{2} \mathrm{CO}_{3}$ & THF & 0 \\
\hline 12 & $\mathrm{PdCl}_{2}\left(\mathrm{PPh}_{3}\right)_{2}$ & L4 & $\mathrm{K}_{2} \mathrm{CO}_{3}$ & THF & 45 \\
\hline 13 & $\mathrm{PdCl}_{2}\left(\mathrm{PPh}_{3}\right)_{2}$ & L5 & $\mathrm{K}_{2} \mathrm{CO}_{3}$ & THF & 8 \\
\hline 14 & $\mathrm{Pd}\left(\mathrm{PPh}_{3}\right)_{4}$ & L4 & $\mathrm{K}_{2} \mathrm{CO}_{3}$ & THF & $54^{\mathrm{d}}$ \\
\hline 15 & $\mathrm{Pd}(\mathrm{OAc})_{2}$ & L4 & $\mathrm{K}_{2} \mathrm{CO}_{3}$ & THF & 18 \\
\hline 16 & {$\left[\mathrm{PdCl}\left(\mathrm{C}_{3} \mathrm{H}_{5}\right)\right]_{2}$} & L4 & $\mathrm{K}_{2} \mathrm{CO}_{3}$ & THF & 23 \\
\hline 17 & $\mathrm{Pd}_{2}(\mathrm{dba})_{3}$ & L4 & $\mathrm{K}_{2} \mathrm{CO}_{3}$ & THF & 26 \\
\hline
\end{tabular}

${ }^{a}$ Reaction conditions: $12(0.1 \mathrm{mmol}), 14(0.15 \mathrm{mmol})$, [Pd] $(0.01 \mathrm{mmol})$, ligand $(0.02 \mathrm{mmol})$. base $(0.175 \mathrm{mmol}), \mathrm{H}_{2} \mathrm{O}(0.15 \mathrm{ml})$, solvent $(3 \mathrm{ml}), 75{ }^{\circ} \mathrm{C}, 1 \mathrm{~h} .{ }^{\mathrm{b}}$ Isolated yield. ${ }^{\mathrm{c}}$ Without $\mathrm{H}_{2} \mathrm{O} .{ }^{\mathrm{d}}$ With the recovery of $\mathbf{1 2}$ in $26 \%$ yield. 
Table S3. Condition screening for Ni(cod) $)_{2}$-mediated cyclization of 8

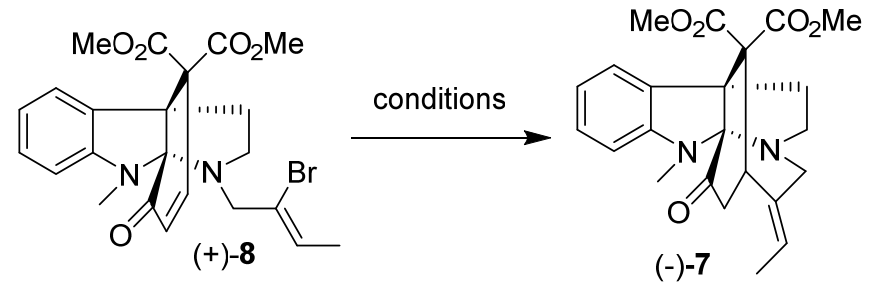

\begin{tabular}{ccc}
\hline entry & conditions & yield \\
\hline 1 & {$\left[\mathrm{Ni}(\mathrm{cod})_{2}\right](5$ equiv $), \mathrm{Et}_{3} \mathrm{~N}(10$ equiv $), \mathrm{MeCN}: \mathrm{DMF}=2: 1$,} & $\mathbf{7}(5 \%)$ \\
& then BHT $(2$ equiv $), 22{ }^{\circ} \mathrm{C}, 1.5 \mathrm{~h}$ & \\
2 & {$\left[\mathrm{Ni}(\mathrm{cod})_{2}\right](2.0$ equiv $), \mathrm{Et}{ }_{3} \mathrm{~N}(4.0$ equiv $), \mathrm{CH}_{3} \mathrm{CN}$,} & $\mathbf{7}$ (trace) \\
3 & then $\mathrm{Et}_{3} \mathrm{SiH}(2.0$ equiv $), 22{ }^{\circ} \mathrm{C}, 1.5 \mathrm{~h}$ & $\mathbf{7}(22 \%)$ \\
4 & {$\left[\mathrm{Ni}(\mathrm{cod})_{2}\right](5$ equiv $), \mathrm{DMF}, 80{ }^{\circ} \mathrm{C}, 2 \mathrm{~h}$} & $\mathbf{7}(40 \%)$ \\
5 & {$\left[\mathrm{Ni}(\mathrm{cod})_{2}\right](6$ equiv $), \mathrm{NaI}(10$ equiv $), \mathrm{Mg}(\mathrm{OTf})_{2}(2$ equiv $), \mathrm{DMF}, 80{ }^{\circ} \mathrm{C}, 2 \mathrm{~h}$} & $\mathbf{7}(50 \%)$ \\
\hline
\end{tabular}

\section{Synthesis and characterizations of unexpected byproducts}
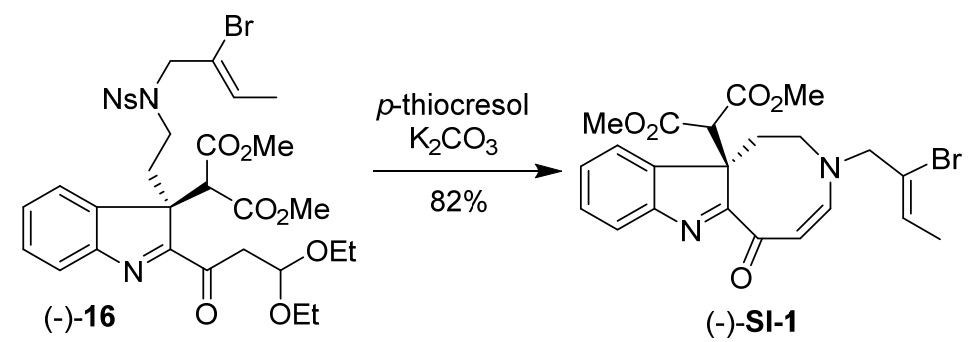

Compound SI-1. To a solution of compound 16 (30 mg, $0.04 \mathrm{mmol})$ in $\mathrm{CH}_{3} \mathrm{CN}$ (3 $\mathrm{mL}$ ) was added $\mathrm{K}_{2} \mathrm{CO}_{2}(16.6 \mathrm{mg}, 0.12 \mathrm{mmol})$ and $p$-thiocresol $(10.0 \mathrm{mg}, 0.08 \mathrm{mmol})$ at room temperature. The solution was stirred at room temperature for $8 \mathrm{~h}$, more $\mathrm{K}_{2} \mathrm{CO}_{2}(8.3 \mathrm{mg}, 0.06 \mathrm{mmol})$ and $p$-thiocresol $(5.0 \mathrm{mg}, 0.04 \mathrm{mmol})$ were then added. The mixture was stirred at room temperature for another $4 \mathrm{~h}$. The reaction was then 
quenched with saturated aqueous $\mathrm{NaHCO}_{3}(5 \mathrm{~mL})$. The resulting mixture was extracted with $\mathrm{CH}_{2} \mathrm{Cl}_{2}(3 \times 5 \mathrm{~mL})$. The combined organic layers were washed with brine $(2 \times 20 \mathrm{~mL})$, dried over anhydrous $\mathrm{Na}_{2} \mathrm{SO}_{4}$, filtered, and concentrated under reduced pressure. The residue was purified by flash column chromatography on silica gel with EtOAc/petroleum ether (2:1) as the eluent to give compound SI-1 (15.5 mg, $82 \%)$ as a yellow foam. SI-1: $[\alpha]_{\mathrm{D}}^{25}=-110.2\left(c=0.4\right.$ in $\left.\mathrm{CHCl}_{3}\right)$; IR (neat): $v\left(\mathrm{~cm}^{-1}\right)$ 2954, 2920, 2855, 1755, 1736, 1628, 1573, 1436, 1404, 1353, 1231, 1178, 1033, 913 , 797, 774, 731; ${ }^{1} \mathrm{H}$ NMR (400 MHz, $\left.\mathrm{CDCl}_{3}\right): \delta 9.92(\mathrm{~d}, J=8.0 \mathrm{~Hz}, 1 \mathrm{H}), 7.91(\mathrm{~d}, J=$ $7.6 \mathrm{~Hz}, 1 \mathrm{H}), 7.73(\mathrm{~d}, J=7.6 \mathrm{~Hz}, 1 \mathrm{H}), 7.45$ (t, $J=7.6 \mathrm{~Hz}, 1 \mathrm{H}), 7.33$ (t, $J=7.6 \mathrm{~Hz}, 1 \mathrm{H})$, $6.04(\mathrm{q}, J=6.4 \mathrm{~Hz}, 1 \mathrm{H}), 5.61(\mathrm{~d}, J=8.0 \mathrm{~Hz}, 1 \mathrm{H}), 4.28(\mathrm{~s}, 1 \mathrm{H}), 4.23(\mathrm{~d}, J=16.4 \mathrm{~Hz}$, $1 \mathrm{H}), 4.18(\mathrm{~d}, J=16.4 \mathrm{~Hz}, 1 \mathrm{H}), 3.88(\mathrm{~s}, 3 \mathrm{H}), 3.58(\mathrm{td}, J=12.0,5.2 \mathrm{~Hz}, 1 \mathrm{H}), 3.47$ (ddd, $J=13.2,7.2,2.0 \mathrm{~Hz}, 1 \mathrm{H}), 3.27$ (s, 3H), $2.96(\mathrm{ddd}, J=14.4,5.2,2.0 \mathrm{~Hz}, 1 \mathrm{H}), 1.80(\mathrm{~d}$, $J=6.4 \mathrm{~Hz}, 3 \mathrm{H}), 1.60(\mathrm{ddd}, J=14.4,11.6,6.8 \mathrm{~Hz}, 1 \mathrm{H}) \mathrm{ppm} ;{ }^{13} \mathrm{C} \mathrm{NMR}(101 \mathrm{MHz}$, $\left.\mathrm{CDCl}_{3}\right): \delta 191.2,173.5,167.4,166.1,154.9,153.4,139.0,129.5,127.6,127.2,125.1$, $122.5,120.3,105.9,58.6,55.2,52.9,52.7,51.2,47.0,30.7,16.5$ ppm; ESI-MS: $\mathrm{m} / \mathrm{z}$ 475.0/477.0 $\left(\mathrm{M}^{+}+\mathrm{H}\right)$; HRMS calcd for $\mathrm{C}_{22} \mathrm{H}_{24} \mathrm{BrN}_{2} \mathrm{O}_{5}[\mathrm{M}+\mathrm{H}]$ : 475.0863, found 475.0862 .

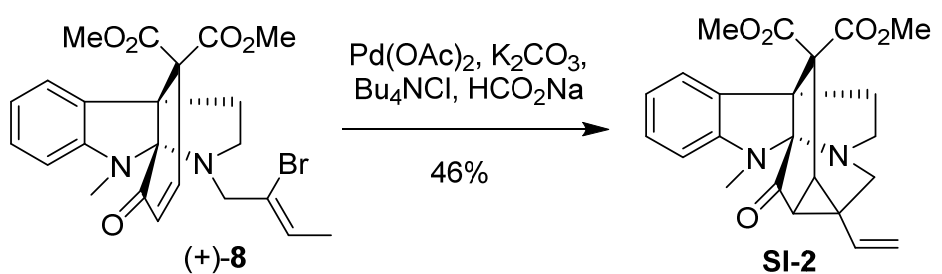

Compound SI-2. To a solution of compound 8 (24.4 mg, $0.05 \mathrm{mmol})$ in DMF (5 mL) was added $\mathrm{K}_{2} \mathrm{CO}_{2}(34.6 \mathrm{mg}, 0.25 \mathrm{mmol}), \mathrm{Bu}_{4} \mathrm{NCl}(34.7 \mathrm{mg}, 0.125 \mathrm{mmol}), \mathrm{HCO}_{2} \mathrm{Na}$ $(4.1 \mathrm{mg}, 0.06 \mathrm{mmol})$ and $\mathrm{Pd}(\mathrm{OAc})_{2}(2.3 \mathrm{mg}, 0.01 \mathrm{mmol})$ at room temperature under argon atmosphere. The mixture was stirred at $80{ }^{\circ} \mathrm{C}$ for $5 \mathrm{~h}$. The reaction was then quenched with saturated aqueous $\mathrm{NaHCO}_{3}(20 \mathrm{~mL})$. The resulting mixture was extracted with $\mathrm{CH}_{2} \mathrm{Cl}_{2}(3 \times 10 \mathrm{~mL})$. The combined organic layers were washed with 
brine $(2 \times 30 \mathrm{~mL})$, dried over anhydrous $\mathrm{Na}_{2} \mathrm{SO}_{4}$, filtered, and concentrated under reduced pressure. The residue was purified by preparative TLC (silica gel, EtOAc/petroleum ether, 1/6) to give vinylcyclopropyl SI-2 (9.5 $\mathrm{mg}, 46 \%)$ as a white foam. SI-2: $[\alpha]_{\mathrm{D}}^{25}=-36.2\left(c=0.1\right.$ in $\left.\mathrm{CHCl}_{3}\right)$; IR (neat): $v\left(\mathrm{~cm}^{-1}\right) 2951,2926,1731$, 1603, 1496, 1471, 1378, 1312, 1259, 1232, 1121, 1091, 991, 914, 877, 737; ${ }^{1} \mathrm{H}$ NMR $\left(400 \mathrm{MHz}, \mathrm{CDCl}_{3}\right): \delta 7.42(\mathrm{dd}, \mathrm{J}=7.6,0.8 \mathrm{~Hz}, 1 \mathrm{H}), 7.02(\mathrm{td}, \mathrm{J}=7.6,1.2 \mathrm{~Hz}, 1 \mathrm{H})$, $6.54(\mathrm{t}, \mathrm{J}=7.6 \mathrm{~Hz}, 1 \mathrm{H}), 6.26(\mathrm{~d}, \mathrm{~J}=7.6 \mathrm{~Hz}, 1 \mathrm{H}), 5.69$ (dd, J = 17.2, $10.4 \mathrm{~Hz}, 1 \mathrm{H})$, $5.27(\mathrm{~d}, \mathrm{~J}=17.2 \mathrm{~Hz}, 1 \mathrm{H}), 5.15(\mathrm{~d}, \mathrm{~J}=10.4 \mathrm{~Hz}, 1 \mathrm{H}), 3.97$ (s, 3H), 3.84 (d, J = 14.0 Hz, 1H), $3.33(\mathrm{~s}, 3 \mathrm{H}), 2.94(\mathrm{~d}, \mathrm{~J}=14.0 \mathrm{~Hz}, 1 \mathrm{H}), 2.83-2.91(\mathrm{~m}, 4 \mathrm{H}), 2.73-2.83(\mathrm{~m}, 1 \mathrm{H})$, $2.62(\mathrm{~d}, \mathrm{~J}=8.8 \mathrm{~Hz}, 1 \mathrm{H}), 2.35(\mathrm{~d}, \mathrm{~J}=8.8 \mathrm{~Hz}, 1 \mathrm{H}), 2.09-2.17(\mathrm{~m}, 2 \mathrm{H}) \mathrm{ppm} ;{ }^{13} \mathrm{C} \mathrm{NMR}$ $\left(101 \mathrm{MHz}, \mathrm{CDCl}_{3}\right): \delta 201.1,170.6,170.2,149.5,138.4,131.2,128.5,122.8,116.2$, $116.1,104.0,93.2,66.9,62.3,56.3,52.9,52.3,50.3,42.6,41.3,39.5,35.0,28.4$ ppm; ESI-MS: m/z $409.2\left(\mathrm{M}^{+}+\mathrm{H}\right)$; HRMS calcd for $\mathrm{C}_{23} \mathrm{H}_{25} \mathrm{~N}_{2} \mathrm{O}_{5}[\mathrm{M}+\mathrm{H}]: 409.1758$, found 409.1758 .

3. Attempted synthesis of pseudoakuammigine and

\section{7-hydroxypseudoakuammigine}

Table S4. Condition screening for the rearrangement of dihydrocorymine

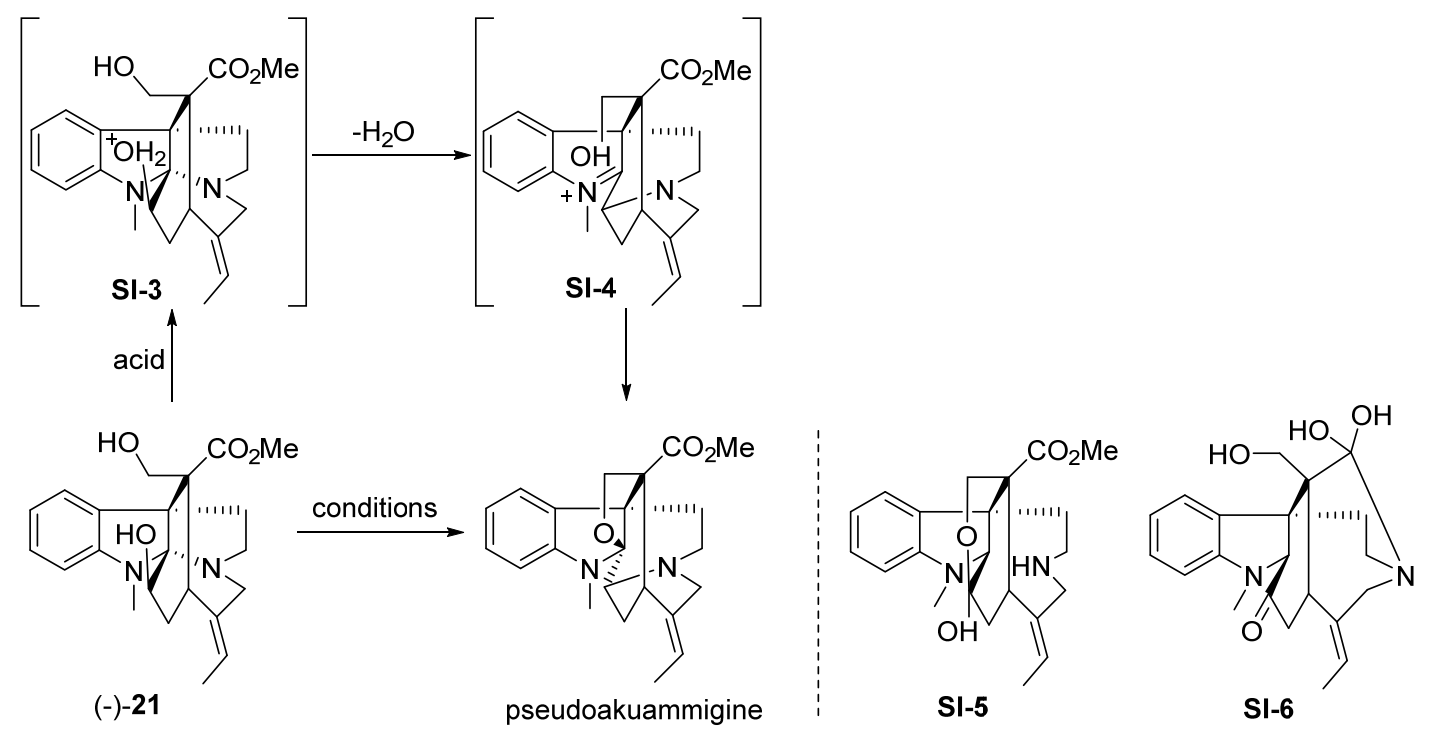




\begin{tabular}{|c|c|c|}
\hline entry & conditions & result $^{\mathrm{b}}$ \\
\hline $1^{\mathrm{a}}$ & $\mathrm{CF}_{3} \mathrm{CO}_{2} \mathrm{H}, \mathrm{rt}$ & SI-5 + SI-6 \\
\hline 2 & $\mathrm{CH}_{3} \mathrm{CO}_{2} \mathrm{H}, \mathrm{rt}$ & s.m. \\
\hline 3 & $\mathrm{CH}_{3} \mathrm{CO}_{2} \mathrm{H}, 60{ }^{\circ} \mathrm{C}$ & SI-5 + SI-6 \\
\hline 4 & $\mathrm{THF} / 3 \mathrm{~N} \mathrm{HCl}(1: 1), \mathrm{rt}$ & s.m. \\
\hline 5 & $\mathrm{THF} / 3 \mathrm{~N} \mathrm{HCl}(1: 1), 60^{\circ} \mathrm{C}$ & SI-5 + SI-6 \\
\hline 6 & $\mathrm{MsOH} / \mathrm{CH}_{2} \mathrm{Cl}_{2}(1: 10), \mathrm{rt}$ & s.m. \\
\hline 7 & $\mathrm{MsOH} / \mathrm{CH}_{2} \mathrm{Cl}_{2}(1: 10), 60{ }^{\circ} \mathrm{C}$ & SI-5 + SI-6 \\
\hline 8 & $\mathrm{TsOH} \cdot \mathrm{H}_{2} \mathrm{O}$ ( 5 equiv), toluene, $\mathrm{rt}$ & s.m. \\
\hline 9 & $\mathrm{TsOH} \cdot \mathrm{H}_{2} \mathrm{O}$ ( 5 equiv), toluene, $60{ }^{\circ} \mathrm{C}$ & SI-5 + SI-6 \\
\hline 12 & $\mathrm{BF}_{3} \cdot \mathrm{Et}_{2} \mathrm{O} / \mathrm{CH}_{2} \mathrm{Cl}_{2}(1: 10), 60{ }^{\circ} \mathrm{C}$ & SI-5 + SI-6 \\
\hline 13 & $\mathrm{Zn}(\mathrm{OTf})_{2}(5$ equiv $), \mathrm{CH}_{2} \mathrm{Cl}_{2}, 60^{\circ} \mathrm{C}$ & SI-5 + SI-6 \\
\hline
\end{tabular}

${ }^{a}$ This reaction condition is same as the Lévy's condition

${ }^{\mathrm{b}}$ compound SI-5 partially decomposed to compound SI-6 during the separation stage, and the yield cannot be analyzed quantitatively.

Table S5. Condition screening for the rearrangement of corymine
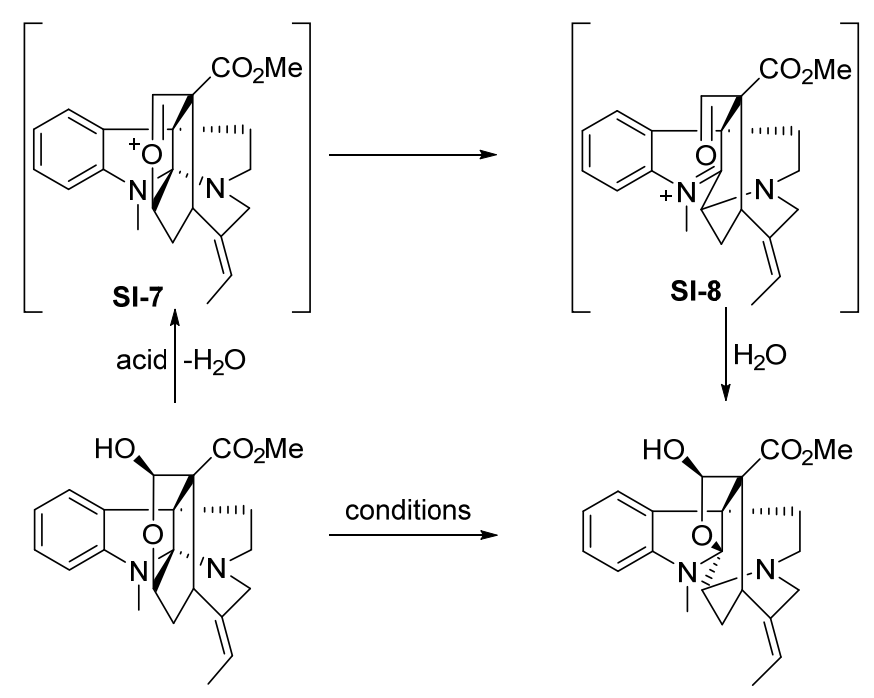

conditions

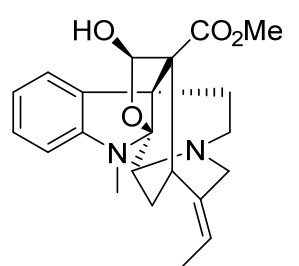

corymine-((+)-5)

17-hydroxypseudoakuammigine

\begin{tabular}{ccc}
\hline entry & conditions & result \\
\hline 1 & $\mathrm{THF} / 3 \mathrm{~N} \mathrm{HCl}(1: 1), \mathrm{rt}$ & s.m. \\
2 & $\mathrm{THF} / 5 \mathrm{~N} \mathrm{HCl}(1: 1), 80^{\circ} \mathrm{C}$ & decomposed \\
\hline
\end{tabular}




\begin{tabular}{|c|c|c|}
\hline 3 & $\mathrm{CF}_{3} \mathrm{CO}_{2} \mathrm{H}, \mathrm{rt}$ & s.m. \\
\hline 4 & $\mathrm{CF}_{3} \mathrm{CO}_{2} \mathrm{H}, 110^{\circ} \mathrm{C}$ & decomposed \\
\hline 5 & $\mathrm{MsOH} / \mathrm{CH}_{2} \mathrm{Cl}_{2}(1: 5), \mathrm{rt}$ & s.m. \\
\hline 6 & $\mathrm{MsOH} / \mathrm{CH}_{2} \mathrm{Cl}_{2}(1: 5), 60{ }^{\circ} \mathrm{C}$ & decomposed \\
\hline 7 & $\mathrm{TsOH} \cdot \mathrm{H}_{2} \mathrm{O}$ ( 3 equiv), toluene, $\mathrm{rt}$ & s.m. \\
\hline 8 & $\mathrm{TsOH} \cdot \mathrm{H}_{2} \mathrm{O}$ ( 3 equiv), toluene, $110^{\circ} \mathrm{C}$ & decomposed \\
\hline 9 & $\mathrm{BF}_{3} \cdot \mathrm{Et}_{2} \mathrm{O}$ ( 3 equiv), $\mathrm{CH}_{2} \mathrm{Cl}_{2}, \mathrm{rt}$ & s.m. \\
\hline 10 & $\mathrm{BF}_{3} \cdot \mathrm{Et}_{2} \mathrm{O}$ ( 3 equiv), $\mathrm{CH}_{2} \mathrm{Cl}_{2}, 60^{\circ} \mathrm{C}$ & decomposed \\
\hline 11 & $\mathrm{BF}_{3} \cdot \mathrm{Et}_{2} \mathrm{O} / \mathrm{CH}_{2} \mathrm{Cl}_{2}(1: 5), \mathrm{rt}$ & decomposed \\
\hline 12 & $\mathrm{Zn}(\mathrm{OTf})_{2}(3$ equiv $), \mathrm{DCE}, 80{ }^{\circ} \mathrm{C}$ & s.m. \\
\hline 13 & $\mathrm{Zn}(\mathrm{OTf})_{2}(3$ equiv $)$, toluene, $110{ }^{\circ} \mathrm{C}$ & decomposed \\
\hline
\end{tabular}

Table S6. Condition screening for the rearrangement of acetylcorymine

conditions
entry $\mathrm{HHF}_{2} \mathrm{~N} \mathrm{HCl}(1: 1), \mathrm{rt}$

${ }^{\mathrm{a}}$ acetylcorymine come from corymine, reaction condition: corymine (1 equiv), $\mathrm{Ac}_{2} \mathrm{O}$ ( 2 equiv), $\mathrm{Cs}_{2} \mathrm{CO}_{3}$ (3 equiv), $\mathrm{CH}_{3} \mathrm{CN}, \mathrm{rt}, 12 \mathrm{~h}$. 
Table S7. Condition screening for the oxidation of SI-10
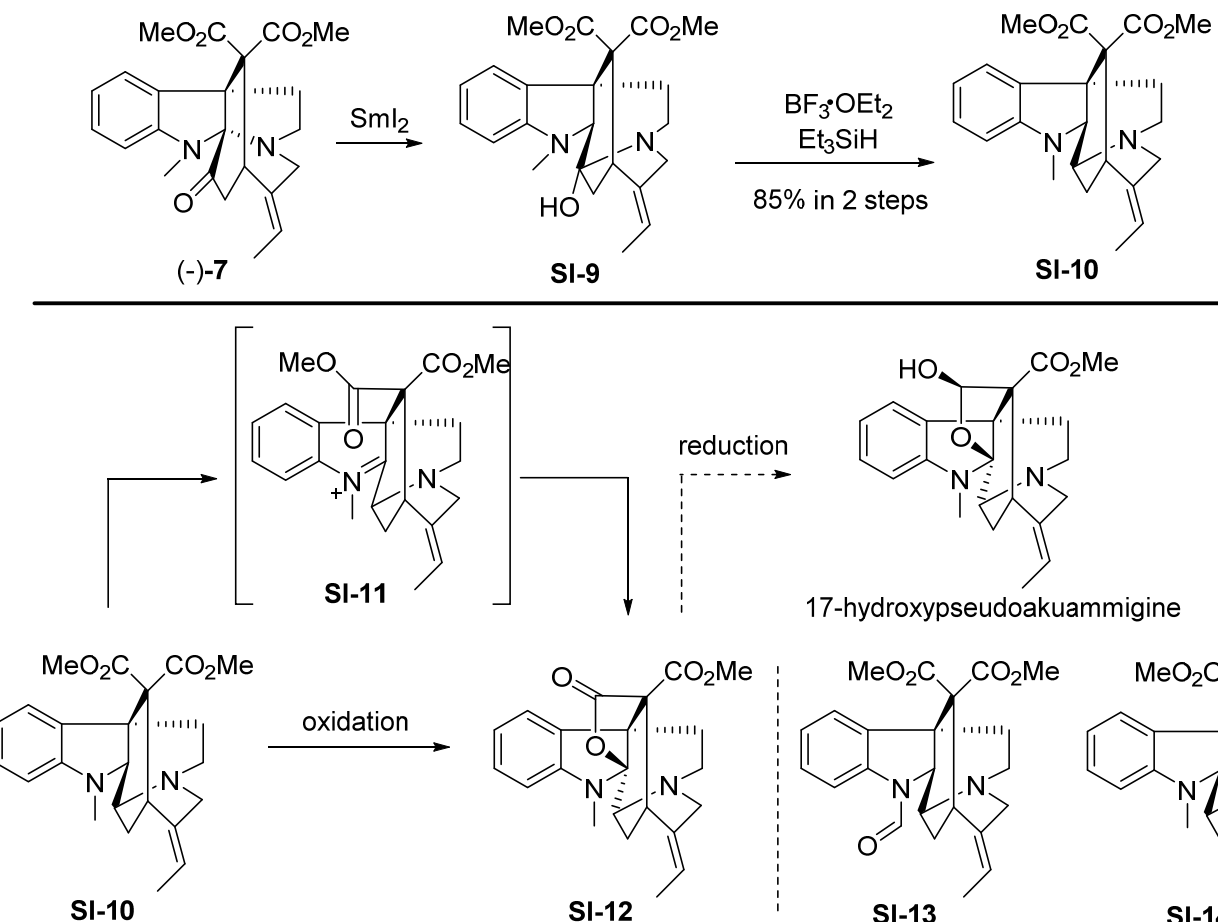

oxidation
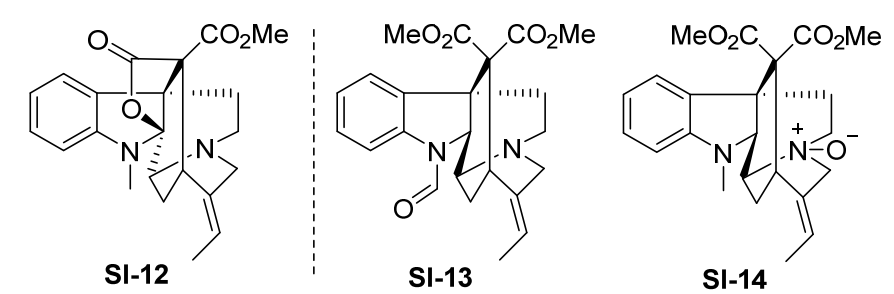

\begin{tabular}{ccc}
\hline entry & conditions & result \\
\hline 1 & $\mathrm{PhIO}(2$ equiv $), \mathrm{CH}_{2} \mathrm{Cl}_{2}, \mathrm{rt}$ & decomposed \\
2 & $\mathrm{MnO}_{2}(100$ equiv $), \mathrm{CH}_{2} \mathrm{Cl}_{2}, \mathrm{rt}$ & $\mathrm{SI}-13(20 \%)$ \\
3 & $\mathrm{PCC}(2$ equiv $), \mathrm{CH}_{2} \mathrm{Cl}_{2}, \mathrm{rt}$ & decomposed \\
4 & $\mathrm{KMnO}_{4}(2$ equiv $), 18$-crown-6 $(2$ equiv $)$, benzene, $\mathrm{rt}$ & decomposed \\
5 & $\mathrm{~Pb}(\mathrm{OAc})_{4}(2$ equiv $), \mathrm{Et}_{3} \mathrm{~N}(1.1$ equiv $), \mathrm{CH}_{2} \mathrm{Cl}_{2}, \mathrm{rt}$ & decomposed \\
6 & $\mathrm{SeO}(2$ equiv $), \mathrm{CH}_{2} \mathrm{Cl}_{2}, \mathrm{rt}$ & s.m. \\
7 & $\mathrm{SeO}(2$ equiv $), \mathrm{CH}_{2} \mathrm{Cl}_{2}, 60{ }^{\circ} \mathrm{C}$ & decomposed \\
8 & $\mathrm{DDQ}(2$ equiv $), \mathrm{toluene}_{2} \mathrm{rt}$ & decomposed \\
9 & $\mathrm{~m}-\mathrm{CPBA}(2$ equiv $), \mathrm{CH}_{2} \mathrm{Cl}_{2}, \mathrm{rt}$ & SI-14 (90\%) \\
\hline
\end{tabular}




\section{Detailed procedures for the synthesis of $(+)$-corymine and related alkaloids}

General Information. All moisture- or oxygen-sensitive reactions were carried out under an argon atmosphere with dry solvents under anhydrous conditions. Tetrahydrofuran (THF) and toluene were distilled from sodium/benzophenone prior to use. Dichloromethane, $N, N$-dimethylformamide (DMF) and acetonitrile were distilled from calcium hydride and stored under an argon atmosphere. Reagents were purchased at the highest commercial quality and used without further purification, unless otherwise stated. All reactions under standard conditions were monitored by thin-layer chromatography (TLC). Silica gel (300 400 mesh), petroleum ether (bp. 60 90 ${ }^{\circ} \mathrm{C}$ ), ethyl acetate, dichloromethane and methanol were used for product purification by flash column chromatography. NMR spectra were recorded on Bruker AV-400 instruments and calibrated using residual non-deuterated solvent as an internal reference. The following abbreviations were used to explain the multiplicities: $\mathrm{s}=$ singlet, $\mathrm{d}=$ doublet, $\mathrm{t}=$ triplet, $\mathrm{q}=$ quartet, $\mathrm{m}=$ multiplet, $\mathrm{br}=$ broad. IR spectra were recorded on a Bio Rad FTS-185 instrument. High-resolution mass spectra (HRMS) were recorded on a Finnigan MAT 8430, or an IonSpec 4.7 Tesla FT mass spectrometer.

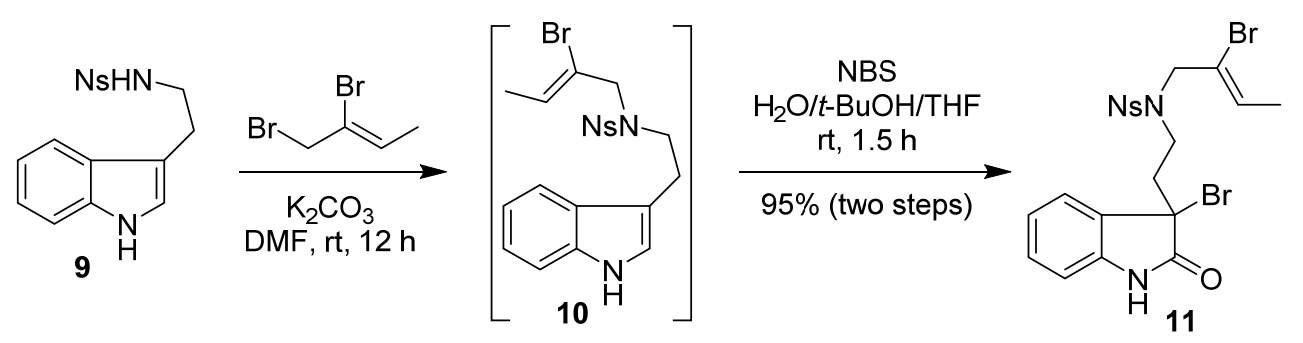

Compound 11. To a solution of $N$-nosyl tryptamine 9 (3.45 g, $10.0 \mathrm{mmol})$ in DMF $(35 \mathrm{~mL})$ was added finely powdered $\mathrm{K}_{2} \mathrm{CO}_{3}(1.66 \mathrm{~g}, 12.0 \mathrm{mmol})$ and (Z)-1,2-Dibromo-2-butene $(3.18 \mathrm{~g}, 15.0 \mathrm{mmol})$ at room temperature under argon 
atmosphere. After stirred at room temperature for $12 \mathrm{~h}$, the reaction was then quenched with saturated aqueous $\mathrm{NaHCO}_{3}(40 \mathrm{~mL})$. The resulting mixture was extracted with $\mathrm{CH}_{2} \mathrm{Cl}_{2}(3 \times 40 \mathrm{~mL})$. The combined organic layers were washed with brine $(2 \times 100 \mathrm{~mL})$, dried over anhydrous $\mathrm{Na}_{2} \mathrm{SO}_{4}$, filtered, and concentrated under reduced pressure to give crude $\mathbf{1 0}$ as a brown oil and directly used for the next reaction. 10: IR (neat): $v\left(\mathrm{~cm}^{-1}\right)$ 3420, 3057, 2920, 2853, 1657, 1620, 1591, 1542, 1457, 1370, 1340, 1230, 1162, 1125, 1098, 954, 910, 852, 735; ${ }^{1} \mathrm{H}$ NMR (400 MHz, $\left.\mathrm{CDCl}_{3}\right): \delta 8.02(\mathrm{br}, 1 \mathrm{H}), 7.92(\mathrm{~d}, J=8.0 \mathrm{~Hz}, 1 \mathrm{H}), 7.56(\mathrm{~d}, J=3.6 \mathrm{~Hz}, 2 \mathrm{H}), 7.41-7.51$ (m, 2H), 7.30 (d, $J=8.0 \mathrm{~Hz}, 1 \mathrm{H}), 7.17$ (t, $J=8.0 \mathrm{~Hz}, 1 \mathrm{H}), 7.09$ (t, $J=8.0 \mathrm{~Hz}, 1 \mathrm{H})$, $6.95(\mathrm{~d}, J=1.6 \mathrm{~Hz}, 1 \mathrm{H}), 5.94$ (q, $J=6.8 \mathrm{~Hz}, 1 \mathrm{H}), 4.29$ (s, 2H), 3.64 (t, $J=7.6 \mathrm{~Hz}$, 2H), 2.97 (t, $J=7.6 \mathrm{~Hz}, 2 \mathrm{H}), 1.73(\mathrm{~d}, J=6.8 \mathrm{~Hz}, 3 \mathrm{H}) \mathrm{ppm} ;{ }^{13} \mathrm{C} \mathrm{NMR}(101 \mathrm{MHz}$, $\left.\mathrm{CDCl}_{3}\right): \delta 147.4,136.1,133.5,133.2,131.5,130.8,128.9,126.9,124.0,122.5,122.4$, $122.0,119.3,118.3,111.7,111.3,55.5,47.0,24.1,16.7$ ppm; ESI-MS: m/z 499.9/501.9 $\left(\mathrm{M}^{+}+\mathrm{Na}\right)$; HRMS calcd for $\mathrm{C}_{20} \mathrm{H}_{21} \mathrm{BrN}_{3} \mathrm{O}_{4} \mathrm{~S}[\mathrm{M}+\mathrm{H}]$ : 478.0431, found 478.0427.

The above tryptamine 10 was re-dissolved in $t-\mathrm{BuOH}(65 \mathrm{~mL})$ and $\mathrm{H}_{2} \mathrm{O}(0.65 \mathrm{~mL})$, a solution of NBS $(3.56 \mathrm{~g}, 20.0 \mathrm{mmol})$ in THF $(65 \mathrm{~mL})$ was then added at room temperature within $1 \mathrm{~h}$. The resulting mixture was stirred at room temperature for 30 min and then concentrated under reduced pressure. Saturated aqueous $\mathrm{Na}_{2} \mathrm{~S}_{2} \mathrm{O}_{3}(50$ $\mathrm{mL})$ was added and the resulting mixture was extracted with $\mathrm{CH}_{2} \mathrm{Cl}_{2}(3 \times 50 \mathrm{~mL})$. The combined organic layers were washed with saturated aqueous $\mathrm{Na}_{2} \mathrm{~S}_{2} \mathrm{O}_{3}(2 \times 150 \mathrm{~mL})$ and brine $(2 \times 150 \mathrm{~mL})$, dried over anhydrous $\mathrm{Na}_{2} \mathrm{SO}_{4}$, filtered, and concentrated under reduced pressure. The residue was purified by flash column chromatography on silica gel with EtOAc/petroleum ether (1:1.5) as the eluent to give 3-bromooxindole $11(5.41 \mathrm{~g}, 95 \%)$ as a white foam. 11: IR (neat): $v\left(\mathrm{~cm}^{-1}\right)$ 3255, 3095, 2916, 1725, 1618, 1543, 1472, 1371, 1265, 1165, 1019, 960, 852, 779; ${ }^{1} \mathrm{H}$ NMR (400 MHz, $\left.\mathrm{CDCl}_{3}\right): \delta 8.93(\mathrm{br}, 1 \mathrm{H}), 7.96(\mathrm{~d}, J=8.0 \mathrm{~Hz}, 1 \mathrm{H}), 7.58-7.71(\mathrm{~m}, 3 \mathrm{H}), 7.34(\mathrm{~d}, J=7.2$ $\mathrm{Hz}, 1 \mathrm{H}), 7.29(\mathrm{td}, J=8.0,0.8 \mathrm{~Hz}, 1 \mathrm{H}), 7.11(\mathrm{t}, J=8.0 \mathrm{~Hz}, 1 \mathrm{H}), 6.93(\mathrm{~d}, J=8.0 \mathrm{~Hz}$, $1 \mathrm{H}), 6.07$ (q, $J=6.4 \mathrm{~Hz}, 1 \mathrm{H}), 4.22(\mathrm{AB}, J=16.8 \mathrm{~Hz}, 2 \mathrm{H}), 3.24-3.46(\mathrm{~m}, 2 \mathrm{H})$, 2.40-2.67 (m, 2H), $1.70(\mathrm{~d}, J=6.4 \mathrm{~Hz}, 3 \mathrm{H}) \mathrm{ppm} ;{ }^{13} \mathrm{C} \mathrm{NMR}\left(101 \mathrm{MHz}, \mathrm{CDCl}_{3}\right): \delta$ 
$175.6,147.8,139.4,133.8,132.9,131.7,131.4,130.6,130.2,129.3,124.7,124.3$, 123.6, 122.0, 111.0, 56.3, 54.0, 43.0, 36.7, 16.8 ppm; ESI-MS: m/z 593.9/595.9/597.9 $\left(\mathrm{M}^{+}+\mathrm{Na}\right)$; HRMS calcd for $\mathrm{C}_{20} \mathrm{H}_{20} \mathrm{Br}_{2} \mathrm{~N}_{3} \mathrm{O}_{5} \mathrm{~S}[\mathrm{M}+\mathrm{H}]$ : 571.9485 , found 571.9478 .

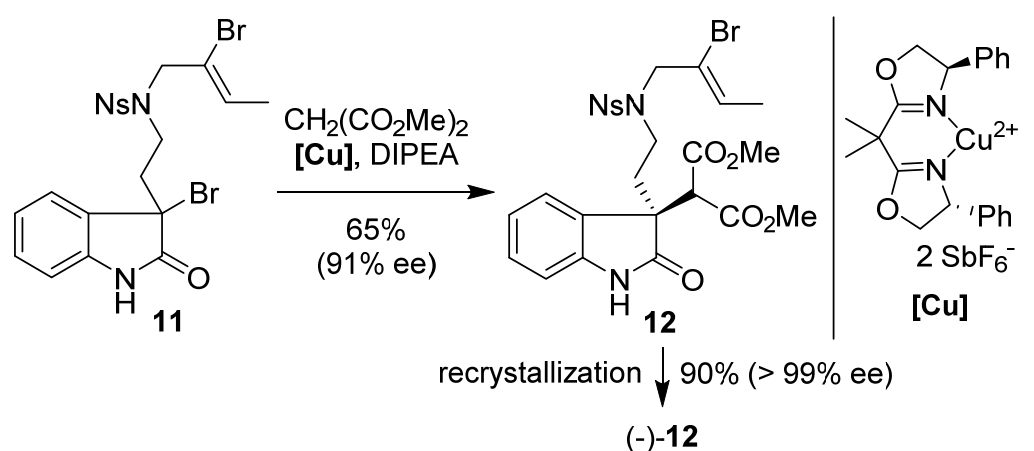

Compound 12. To a reaction tube equipped with a stirbar was added [Cu] $(1.31 \mathrm{~g}$, $1.5 \mathrm{mmol})$ and $3 \AA$ MS $(1.62 \mathrm{~g})$ in the glovebox. After the reaction tube was removed from the glovebox, $\mathrm{CH}_{2} \mathrm{Cl}_{2}(50 \mathrm{~mL})$ was added and allowed to stir at room temperature for 15 minutes. Upon cooling to $-20{ }^{\circ} \mathrm{C}$, malonate $(1.72 \mathrm{~mL}, 15 \mathrm{mmol})$ followed by 3-bromooxindole $11(2.86 \mathrm{~g}, 5.0 \mathrm{mmol})$ dissolved in $\mathrm{CH}_{2} \mathrm{Cl}_{2}(25 \mathrm{~mL})$ were added. The solution was stirred at $-20{ }^{\circ} \mathrm{C}$ for $5 \mathrm{~min}$, DIPEA $(1.66 \mathrm{~mL}, 10 \mathrm{mmol})$ was added. After stirred at $-20{ }^{\circ} \mathrm{C}$ for $72 \mathrm{~h}$, the reaction was then quenched with saturated aqueous $\mathrm{NH}_{4} \mathrm{Cl}(75 \mathrm{~mL})$. The resulting mixture was extracted with $\mathrm{CH}_{2} \mathrm{Cl}_{2}$ $(3 \times 75 \mathrm{~mL})$. The combined organic layers were washed with brine $(2 \times 200 \mathrm{~mL})$, dried over anhydrous $\mathrm{Na}_{2} \mathrm{SO}_{4}$, filtered, and concentrated under reduced pressure. The residue was purified by flash column chromatography on silica gel with EtOAc/petroleum ether (1:1.5) as the eluent to give lactam $12(2.02 \mathrm{~g}, 65 \%, 91 \%$ ee $)$ as a white foam. This white foam was recrystallized from EtOAc/petroleum ether (70 $\mathrm{mL}, 1: 10)$ to give the essentially enantiopure product $12(1.82 \mathrm{~g}, 90 \%, 99 \%$ ee) from the mother liquid. 12: $[\alpha]_{\mathrm{D}}^{25}=-5.6\left(c=1.0\right.$ in $\left.\mathrm{CHCl}_{3}\right)$; IR (neat): $v\left(\mathrm{~cm}^{-1}\right) 3350,3094$, 2953, 1756, 1733, 1619, 1544, 1472, 1370, 1199, 1164, 1023, 912, 852, 736; ${ }^{1} \mathrm{H}$ NMR (400 MHz, $\left.\mathrm{CDCl}_{3}\right): \delta 8.76$ (br, 1H), 7.79-7.85 (m, 1H), 7.56-7.69 (m, 3H), 7.45 (d, J $=7.6 \mathrm{~Hz}, 1 \mathrm{H}), 7.24(\mathrm{t}, J=7.6 \mathrm{~Hz}, 1 \mathrm{H}), 7.04(\mathrm{t}, J=7.6 \mathrm{~Hz}, 1 \mathrm{H}), 6.88(\mathrm{~d}, J=7.6 \mathrm{~Hz}$, 
$1 \mathrm{H}), 5.90(\mathrm{q}, J=6.4 \mathrm{~Hz}, 1 \mathrm{H}), 4.13(\mathrm{~d}, J=15.6 \mathrm{~Hz}, 1 \mathrm{H}), 4.07(\mathrm{~d}, J=15.6 \mathrm{~Hz}, 1 \mathrm{H})$, $4.03(\mathrm{~s}, 1 \mathrm{H}), 3.75(\mathrm{~s}, 3 \mathrm{H}), 3.47(\mathrm{~s}, 3 \mathrm{H}), 3.05-3.18(\mathrm{~m}, 1 \mathrm{H}), 2.73-2.87(\mathrm{~m}, 1 \mathrm{H}), 2.15(\mathrm{t}$, $J=8.4 \mathrm{~Hz}, 2 \mathrm{H}), 1.67(\mathrm{~d}, J=6.4 \mathrm{~Hz}, 3 \mathrm{H}) \mathrm{ppm} ;{ }^{13} \mathrm{C} \mathrm{NMR}\left(101 \mathrm{MHz}, \mathrm{CDCl}_{3}\right): \delta 178.5$, $167.6,166.7,147.7,141.2,133.5,133.0,131.7,131.6,129.8,129.0,128.4,125.1$, 124.2, 122.9, 122.3, 109.9, 56.5, 56.3, 52.7, 52.6, 50.7, 41.5, 33.3, 16.8 ppm; ESI-MS: $\mathrm{m} / \mathrm{z}$ 646.0/648.0 $\left(\mathrm{M}^{+}+\mathrm{Na}\right)$; HRMS calcd for $\mathrm{C}_{25} \mathrm{H}_{27} \mathrm{BrN}_{3} \mathrm{O}_{9} \mathrm{~S}[\mathrm{M}+\mathrm{H}]:$ 624.0646, found 624.0639.

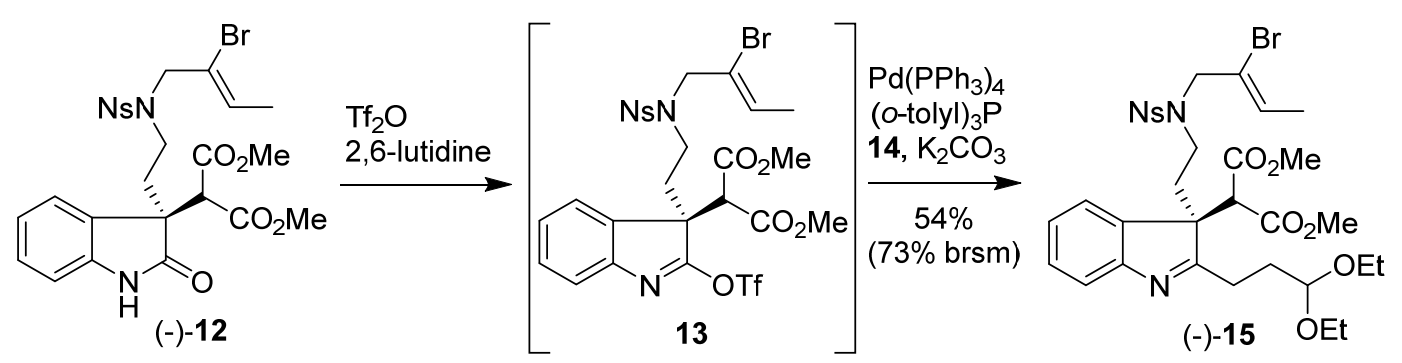

Compound 15. To a solution of lactam $12(0.5 \mathrm{~g}, 0.8 \mathrm{mmol})$ in $\mathrm{CH}_{2} \mathrm{Cl}_{2}(10 \mathrm{~mL})$ was added 2,6-lutidine $(0.33 \mathrm{~mL}, 2.8 \mathrm{mmol})$ and $\mathrm{Tf}_{2} \mathrm{O}(0.34 \mathrm{~mL}, 2.0 \mathrm{mmol})$ at $-78{ }^{\circ} \mathrm{C}$ under argon atmosphere. After stirred at $-78{ }^{\circ} \mathrm{C}$ for $2 \mathrm{~h}$, the reaction was then quenched with $1 \mathrm{~N} \mathrm{HCl}(10 \mathrm{~mL})$. The resulting mixture was extracted with $\mathrm{CH}_{2} \mathrm{Cl}_{2}$ (3 $\times 10 \mathrm{~mL})$. The combined organic layers were washed with $1 \mathrm{~N} \mathrm{HCl}(2 \times 30 \mathrm{~mL})$ and saturated aqueous $\mathrm{NaHCO}_{3}(2 \times 30 \mathrm{~mL})$, dried over anhydrous $\mathrm{Na}_{2} \mathrm{SO}_{4}$, filtered, and concentrated under reduced pressure. The residue was purified by flash column chromatography on silica gel with EtOAc/petroleum ether (1:3) as the eluent to give the corresponding triflate 13. (After the column chromatography, the solvent must not completely be removed. If the solvent completely concentrated, triflate $\mathbf{1 3}$ was decomposed to lactam 12). A solution of triflate 13 in eluent ( $5 \mathrm{~mL})$ was concentrated under high vacuum and backfilled with argon, degassed THF (10 ml) was then added via syringe. A solution of triflate $\mathbf{1 3}$ in THF was prepared for the next step. A solution of finely powdered $\mathrm{K}_{2} \mathrm{CO}_{3}(194 \mathrm{mg}, 1.4 \mathrm{mmol})$ in degassed THF (15 mL) and $\mathrm{H}_{2} \mathrm{O}(1.25 \mathrm{~mL})$ stirred at $75{ }^{\circ} \mathrm{C}$ under argon atmosphere for $5 \mathrm{~min}, \mathrm{Pd}\left(\mathrm{PPh}_{3}\right)_{4}(93$ 
$\mathrm{mg}, 0.08 \mathrm{mmol}),(\mathrm{o}-$ Tolyl) 3 P $(49 \mathrm{mg}, 0.16 \mathrm{mmol})$, borane $14(0.5 \mathrm{M}$ solution in THF, $2.4 \mathrm{ml}, 1.2 \mathrm{mmol})$ and the solution of triflate $\mathbf{1 3}$ in THF (10 ml) above were added successively. The resulting mixture was stirred at $75^{\circ} \mathrm{C}$ for $1 \mathrm{~h}$ and then cooled down to room temperature. The reaction was then quenched with saturated aqueous $\mathrm{NaHCO}_{3}(25 \mathrm{~mL})$. The resulting mixture was extracted with $\mathrm{CH}_{2} \mathrm{Cl}_{2}(3 \times 25 \mathrm{~mL})$. The combined organic layers were washed with brine $(2 \times 100 \mathrm{~mL})$, dried over anhydrous $\mathrm{Na}_{2} \mathrm{SO}_{4}$, filtered, and concentrated under reduced pressure. The residue was purified by flash column chromatography on silica gel with EtOAc/petroleum ether (1:2) as the eluent to give indolenine $15(320 \mathrm{mg}, 54 \%, 73 \% \mathrm{brsm})$ as a white foam. 15: $[\alpha]_{\mathrm{D}}^{25}$ $=-48.2\left(c=1.0\right.$ in $\left.\mathrm{CHCl}_{3}\right)$; IR (neat): $v\left(\mathrm{~cm}^{-1}\right) 2972,2927,1756,1737,1658,1578$, 1545, 1438, 1371, 1351, 1262, 1164, 1059, 957, 852, 774; ${ }^{1} \mathrm{H}$ NMR (400 MHz, $\left.\mathrm{CDCl}_{3}\right): \delta 7.81(\mathrm{dd}, J=7.6,1.2 \mathrm{~Hz}, 1 \mathrm{H}), 7.54-7.68(\mathrm{~m}, 4 \mathrm{H}), 7.49(\mathrm{~d}, J=7.6 \mathrm{~Hz}, 1 \mathrm{H})$, $7.33(\mathrm{td}, J=7.6,0.8 \mathrm{~Hz}, 1 \mathrm{H}), 7.21(\mathrm{t}, J=7.6 \mathrm{~Hz}, 1 \mathrm{H}), 5.77$ (q, $J=6.4 \mathrm{~Hz}, 1 \mathrm{H}), 4.69$ (t, $J=5.2 \mathrm{~Hz}, 1 \mathrm{H}), 4.05(\mathrm{~d}, J=15.6 \mathrm{~Hz}, 1 \mathrm{H}), 3.96(\mathrm{~s}, 1 \mathrm{H}), 3.94(\mathrm{~d}, J=15.6 \mathrm{~Hz}, 1 \mathrm{H})$, 3.66-3.78 (m, 5H), 3.50-3.63 (m, 2H), 3.38 (s, 3H), 2.48-2.76 (m, 4H), 2.09-2.37 (m, 3H), 1.91-2.03 (m, 1H), $1.70(\mathrm{~d}, J=6.4 \mathrm{~Hz}, 3 \mathrm{H}), 1.23(\mathrm{td}, J=6.8,3.2 \mathrm{~Hz}, 6 \mathrm{H})$ ppm; ${ }^{13} \mathrm{C}$ NMR (101 MHz, $\left.\mathrm{CDCl}_{3}\right): \delta 184.3,167.1,166.0,155.1,147.7,137.4,133.5$, 132.5, 131.7, 131.6, 129.7, 128.9, 125.5, 124.1 (two peaks), 122.9, 120.2, 102.3, 61.9, 61.4, 59.8, 57.4, 55.7, 52.6, 52.4, 42.0, 32.4, 29.7, 24.5, 16.8, 15.4, 15.3 ppm; ESI-MS: $m / z$ 760.1/762.1 $\left(\mathrm{M}^{+}+\mathrm{Na}\right)$; HRMS calcd for $\mathrm{C}_{32} \mathrm{H}_{41} \mathrm{BrN}_{3} \mathrm{O}_{10} \mathrm{~S}[\mathrm{M}+\mathrm{H}]$ : 738.1691, found 738.1680 .

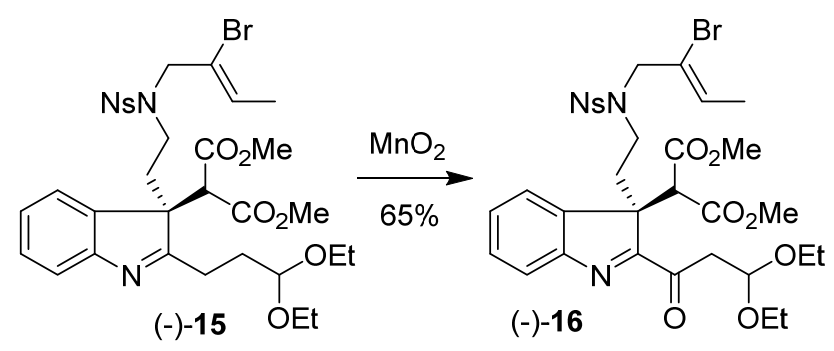

Compound 16. To a solution of indolenine $15(300 \mathrm{mg}, 0.41 \mathrm{mmol})$ in $\mathrm{CH}_{2} \mathrm{Cl}_{2}(30$ $\mathrm{mL})$ was added $\mathrm{MnO}_{2}(3.56 \mathrm{~g}, 41 \mathrm{mmol})$ at room temperature. The solution was 
stirred at room temperature for $24 \mathrm{~h} . \mathrm{MnO}_{2}$ was removed via filtration through a pad of Celite ${ }^{\circledR}$. The filtrate was then concentrated under reduced pressure. The residue was purified by flash column chromatography on silica gel with EtOAc/petroleum ether $(1: 4)$ as the eluent to give ketone $16(198 \mathrm{mg}, 65 \%)$ as a white foam. 16: $[\alpha]_{\mathrm{D}}^{25}=$ $-29.3\left(c=1.0\right.$ in $\left.\mathrm{CHCl}_{3}\right)$; IR (neat): $v\left(\mathrm{~cm}^{-1}\right)$ 3073, 2977, 2953, 1756, 1737, 1682, 1589, 1544, 1437, 1371, 1277, 1166, 1059, 955, 911, 852, 775, 736; ${ }^{1} \mathrm{H}$ NMR (400 $\left.\mathrm{MHz}, \mathrm{CDCl}_{3}\right): \delta 7.76(\mathrm{~d}, J=7.6 \mathrm{~Hz}, 1 \mathrm{H}), 7.73(\mathrm{~d}, J=7.6 \mathrm{~Hz}, 1 \mathrm{H}), 7.63-7.70(\mathrm{~m}, 2 \mathrm{H})$, 7.55-7.62 (m, 2H), 7.48 (td, $J=7.6,1.2 \mathrm{~Hz}, 1 \mathrm{H}), 7.42$ (td, $J=7.6,1.2 \mathrm{~Hz}, 1 \mathrm{H}), 5.80$ (q, $J=6.4 \mathrm{~Hz}, 1 \mathrm{H}), 5.19(\mathrm{t}, J=5.6 \mathrm{~Hz}, 1 \mathrm{H}), 4.50(\mathrm{~s}, 1 \mathrm{H}), 4.07(\mathrm{~d}, J=15.2 \mathrm{~Hz}, 1 \mathrm{H})$, $3.99(\mathrm{~d}, J=15.2 \mathrm{~Hz}, 1 \mathrm{H}), 3.65-3.77(\mathrm{~m}, 5 \mathrm{H}), 3.53-3.65(\mathrm{~m}, 2 \mathrm{H}), 3.47$ (dd, $J=16.0$, $5.6 \mathrm{~Hz}, 1 \mathrm{H}), 3.39(\mathrm{dd}, J=16.0,5.6 \mathrm{~Hz}, 1 \mathrm{H}), 3.33(\mathrm{~s}, 3 \mathrm{H}), 2.50-2.62(\mathrm{~m}, 2 \mathrm{H})$, 2.37-2.49 (m, 1H), 2.03-2.15 (m, 1H), 1.69 (d, $J=6.4 \mathrm{~Hz}, 3 \mathrm{H}), 1.18(\mathrm{td}, J=6.8,3.6$ $\mathrm{Hz}, 6 \mathrm{H}) \mathrm{ppm} ;{ }^{13} \mathrm{C} \mathrm{NMR}\left(101 \mathrm{MHz}, \mathrm{CDCl}_{3}\right): \delta 195.3,173.9,167.3,166.2,153.9$, $147.7,139.7,133.4,133.0,131.7,131.6,129.7,129.5,129.3,124.9,124.1,123.8$, $122.5,99.0,61.4,61.3,58.8,56.4,55.4,52.6,52.3,43.6,41.1,32.0,16.8,15.2$ (overlap) ppm; ESI-MS: $\mathrm{m} / \mathrm{z} \quad 774.0 / 776.0 \quad\left(\mathrm{M}^{+}+\mathrm{Na}\right)$; HRMS calcd for $\mathrm{C}_{32} \mathrm{H}_{38} \mathrm{BrN}_{3} \mathrm{O}_{11} \mathrm{SNa}[\mathrm{M}+\mathrm{Na}]:$ 774.1303, found 774.1294.

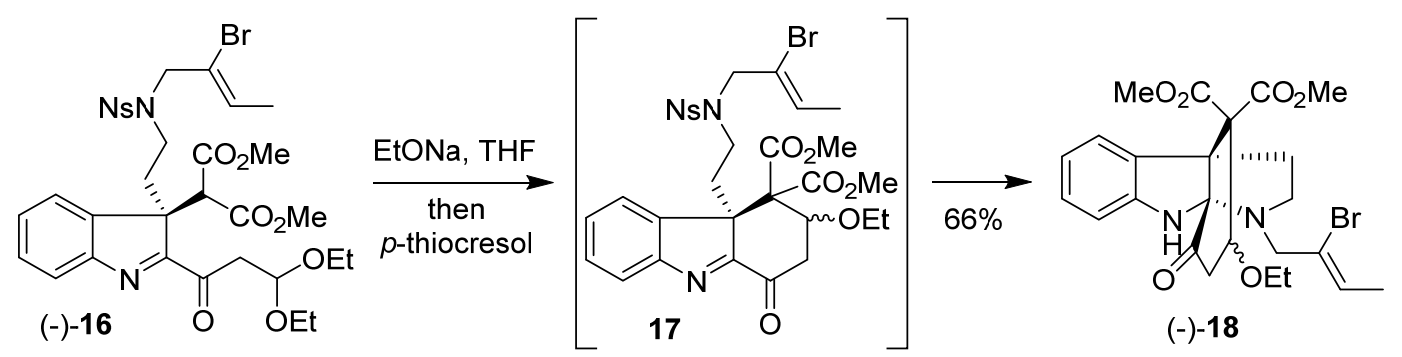

Compound 18. To a solution of ketone 16 (180 mg, $0.24 \mathrm{mmol})$ in THF (27 mL) was added EtONa (65.3 $\mathrm{mg}, 0.96 \mathrm{mmol})$ in 2 portions with one hour interval at room temperature under argon atmosphere, $p$-thiocresol $(120 \mathrm{mg}, 0.96 \mathrm{mmol})$ was then directly added. The mixture was stirred at room temperature for another $10 \mathrm{~min}$. The reaction was then quenched with saturated aqueous $\mathrm{NH}_{4} \mathrm{Cl}(20 \mathrm{~mL})$. The resulting mixture was extracted with $\mathrm{CH}_{2} \mathrm{Cl}_{2}(3 \times 20 \mathrm{~mL})$. The combined organic layers were 
washed with brine $(2 \times 60 \mathrm{~mL})$, dried over anhydrous $\mathrm{Na}_{2} \mathrm{SO}_{4}$, filtered, and concentrated under reduced pressure. The residue was purified by flash column chromatography on silica gel with EtOAc/ $\mathrm{CH}_{2} \mathrm{Cl}_{2} /$ petroleum ether $(1: 4: 20)$ as the eluent to give tetracyclic $18(82 \mathrm{mg}, 65 \%)$ as a white foam. 18: $[\alpha]_{\mathrm{D}}^{25}=-145.6(c=$ 1.0 in $\mathrm{CHCl}_{3}$ ); IR (neat): $v\left(\mathrm{~cm}^{-1}\right) 3357,2975,2951,2830,1742,1724,1605,1592$, 1486, 1435, 1356, 1256, 1200, 1090, 912, 815, 748, 733; ${ }^{1} \mathrm{H}$ NMR (400 MHz, $\left.\mathrm{CDCl}_{3}\right)$ : $\delta$ major $7.57(\mathrm{~d}, J=7.6 \mathrm{~Hz}, 1 \mathrm{H}), 7.03(\mathrm{t}, J=7.6 \mathrm{~Hz}, 1 \mathrm{H}), 6.70(\mathrm{t}, J=7.6 \mathrm{~Hz}, 1 \mathrm{H})$, $6.63(\mathrm{~d}, J=7.6 \mathrm{~Hz}, 1 \mathrm{H}), 5.87$ (q, $J=6.4 \mathrm{~Hz}, 1 \mathrm{H}), 5.08(\mathrm{~s}, 1 \mathrm{H}), 4.77$ (t, $J=8.4 \mathrm{~Hz}$, $1 \mathrm{H}), 3.94(\mathrm{~s}, 3 \mathrm{H}), 3.60-3.76(\mathrm{~m}, 2 \mathrm{H}), 3.38-3.55(\mathrm{~m}, 1 \mathrm{H}), 3.25-3.36(\mathrm{~m}, 4 \mathrm{H})$, 3.06-3.13 (m, 1H), 2.90-3.02 (m, 1H), 2.47 (td, $J=11.6,7.2 \mathrm{~Hz}, 1 \mathrm{H}), 2.26-2.39(\mathrm{~m}$, 2H), $1.86(\mathrm{dd}, J=12.4,5.6 \mathrm{~Hz}, 1 \mathrm{H}), 1.74(\mathrm{dd}, J=6.4,1.2 \mathrm{~Hz}, 3 \mathrm{H}), 1.14-1.23(\mathrm{~m}, 3 \mathrm{H})$ ppm; minor $7.54(\mathrm{~d}, J=7.6 \mathrm{~Hz}, 1 \mathrm{H}), 6.99(\mathrm{t}, J=7.6 \mathrm{~Hz}, 1 \mathrm{H}), 6.70(\mathrm{t}, J=7.6 \mathrm{~Hz}, 1 \mathrm{H})$, $6.55(\mathrm{~d}, J=7.6 \mathrm{~Hz}, 1 \mathrm{H}), 5.87(\mathrm{q}, J=6.4 \mathrm{~Hz}, 1 \mathrm{H}), 4.96(\mathrm{~s}, 1 \mathrm{H}), 4.42(\mathrm{dd}, J=8.8,2.4$ $\mathrm{Hz}, 1 \mathrm{H}), 3.81(\mathrm{~s}, 3 \mathrm{H}), 3.60-3.76(\mathrm{~m}, 1 \mathrm{H}), 3.38-3.55(\mathrm{~m}, 2 \mathrm{H}), 3.25-3.36(\mathrm{~m}, 1 \mathrm{H})$, 3.13-3.23 (m, 1H), 3.06-3.13 (m, 1H), 3.02-3.07 (m, 4H), 2.90-3.02 (m, 1H), 2.62 $(\mathrm{dd}, J=12.8,4.8 \mathrm{~Hz}, 1 \mathrm{H}), 2.26-2.39(\mathrm{~m}, 1 \mathrm{H}), 1.72(\mathrm{dd}, J=6.4,1.2 \mathrm{~Hz}, 3 \mathrm{H})$, 1.14-1.23 (m, 3H) ppm; ${ }^{13} \mathrm{C}$ NMR (101 $\mathrm{MHz}, \mathrm{CDCl}_{3}$ ) (extra peaks due to diastereoisomers): $\delta$ 203.5, 202.7, 169.9, 169.3, 168.5 (two peaks), 150.5, 149.5, $132.1,131.3,128.6,128.4,127.3,126.9,126.5,125.9,124.7,124.5,119.7,119.2$, 110.2, 109.6, 90.4, 87.6, 77.6, 72.6, 66.0, 65.8, 65.6, 64.9, 64.4, 62.7, 56.5, 55.9, 52.3, 52.1 (two peaks), 51.8, 50.2, 48.6, 40.8, 39.2, 37.6, 37.4, 16.6 (two peaks), 15.4, 15.3 ppm; ESI-MS: m/z 521.1/523.1 $\left(\mathrm{M}^{+}+\mathrm{H}\right)$; HRMS calcd for $\mathrm{C}_{24} \mathrm{H}_{30} \mathrm{BrN}_{2} \mathrm{O}_{6}[\mathrm{M}+\mathrm{H}]$ : 521.1282 , found 521.1279 .

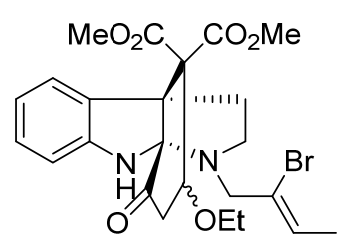

(-)-18

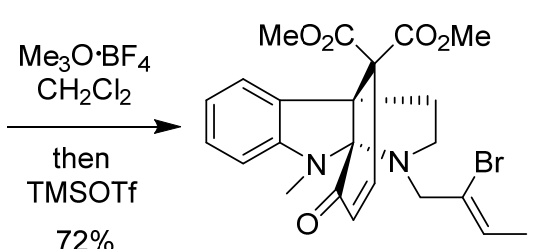

(+)-8

Compound 8. To a solution of tetracyclic $18(73 \mathrm{mg}, 0.14 \mathrm{mmol})$ in $\mathrm{CH}_{2} \mathrm{Cl}_{2}(15 \mathrm{~mL})$ 
was added added $\mathrm{Me}_{3} \mathrm{O} \cdot \mathrm{BF}_{4}(104 \mathrm{mg}, 0.7 \mathrm{mmol})$ at room temperature under argon atmosphere. The solution was stirred at room temperature for $24 \mathrm{~h}$, TMSOTf $(0.26$ $\mathrm{mL}, 1.4 \mathrm{mmol}$ ) was then directly added. The mixture was stirred at room temperature for another $24 \mathrm{~h}$. The reaction was quenched with $\mathrm{Et}_{3} \mathrm{~N}(0.3 \mathrm{~mL}, 2.1 \mathrm{mmol})$. Saturated aqueous $\mathrm{NaHCO}_{3}(15 \mathrm{~mL})$ was then added and the resulting mixture was extracted with $\mathrm{CH}_{2} \mathrm{Cl}_{2}(3 \times 15 \mathrm{~mL})$. The combined organic layers were washed with brine $(2 \times$ $50 \mathrm{~mL}$ ), dried over anhydrous $\mathrm{Na}_{2} \mathrm{SO}_{4}$, filtered, and concentrated under reduced pressure. The residue was purified by flash column chromatography on silica gel with EtOAc/petroleum ether (1:10) as the eluent to give olefin 8 (49 $\mathrm{mg}, 72 \%)$ as a yellow foam. 8: $[\alpha]_{\mathrm{D}}^{25}=+42.0\left(c=1.0\right.$ in $\left.\mathrm{CHCl}_{3}\right)$; IR (neat): $v\left(\mathrm{~cm}^{-1}\right) 3051,3002,2820,1758$, 1736, 1686, 1601, 1492, 1434, 1367, 1254, 1215, 1155, 1109, 933, 814, 746; ${ }^{1} \mathrm{H}$ NMR $\left(400 \mathrm{MHz}, \mathrm{CDCl}_{3}\right): \delta 7.63(\mathrm{dd}, J=7.6,0.8 \mathrm{~Hz}, 1 \mathrm{H}), 7.12(\mathrm{td}, J=7.6,0.8 \mathrm{~Hz}, 1 \mathrm{H})$, $6.99(\mathrm{~d}, J=10.4 \mathrm{~Hz}, 1 \mathrm{H}), 6.64(\mathrm{td}, J=7.6,0.8 \mathrm{~Hz}, 1 \mathrm{H}), 6.38(\mathrm{~d}, J=10.4 \mathrm{~Hz}, 1 \mathrm{H})$, $6.36(\mathrm{~d}, J=7.6 \mathrm{~Hz}, 1 \mathrm{H}), 5.84(\mathrm{q}, J=6.4 \mathrm{~Hz}, 1 \mathrm{H}), 4.12(\mathrm{dt}, J=13.6,1.6 \mathrm{~Hz}, 1 \mathrm{H}), 3.90$ (s, 3H), $3.25(\mathrm{~s}, 3 \mathrm{H}), 3.23(\mathrm{~d}, J=13.6 \mathrm{~Hz}, 1 \mathrm{H}), 3.21(\mathrm{~s}, 3 \mathrm{H}), 2.91(\mathrm{dd}, J=8.4,6.4 \mathrm{~Hz}$, 1H), $2.61(\mathrm{dd}, J=12.0,4.4 \mathrm{~Hz}, 1 \mathrm{H}), 2.29(\mathrm{ddd}, J=12.0,8.4,4.4 \mathrm{~Hz}, 1 \mathrm{H}), 2.05(\mathrm{td}, J$ $=12.0,6.8 \mathrm{~Hz}, 1 \mathrm{H}), 1.70(\mathrm{dd}, J=6.4,1.6 \mathrm{~Hz}, 3 \mathrm{H}) \mathrm{ppm} ;{ }^{13} \mathrm{C} \mathrm{NMR}\left(101 \mathrm{MHz}, \mathrm{CDCl}_{3}\right)$ : $\delta 188.0,168.6,167.6,152.5,139.2,131.9,129.4,126.9,126.7,126.2,125.0,117.1$, 105.1, 86.0, 64.5, 64.2, 56.1, 53.2, 53.0, 48.1, 37.8, 32.7, 16.6 ppm; ESI-MS: m/z 489.0/491.0 $\left(\mathrm{M}^{+}+\mathrm{H}\right)$; HRMS calcd for $\mathrm{C}_{23} \mathrm{H}_{26} \mathrm{BrN}_{2} \mathrm{O}_{5}[\mathrm{M}+\mathrm{H}]$ : 489.1020, found 489.1017 .

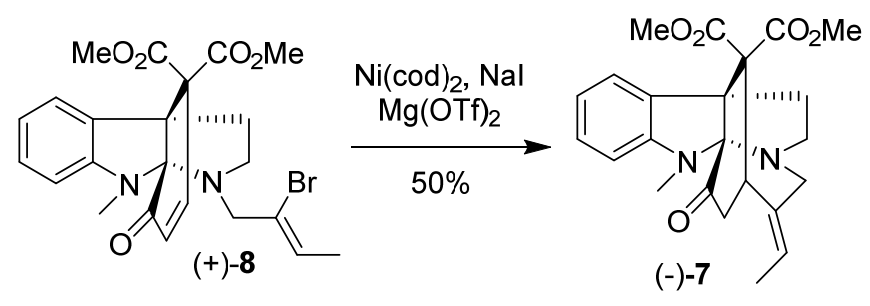

Compound 7. To a solution of olefin $8(30 \mathrm{mg}, 0.062 \mathrm{mmol})$ in DMF $(9 \mathrm{~mL})$ was successively added $\mathrm{NaI}$ (93 mg, $0.62 \mathrm{mmol}), \mathrm{Mg}(\mathrm{OTf})_{2}(40 \mathrm{mg}, 0.124 \mathrm{mmol})$ and $\mathrm{Ni}(\operatorname{cod})_{2}(103 \mathrm{mg}, 0.372 \mathrm{mmol})$ in glovebox. The mixture was stirred at $80{ }^{\circ} \mathrm{C}$ for $2 \mathrm{~h}$. 
The reaction was then quenched with saturated aqueous $\mathrm{NaHCO}_{3}(20 \mathrm{~mL})$. The resulting mixture was extracted with $\mathrm{CH}_{2} \mathrm{Cl}_{2}(3 \times 10 \mathrm{~mL})$. The combined organic layers were washed with brine $(2 \times 30 \mathrm{~mL})$, dried over anhydrous $\mathrm{Na}_{2} \mathrm{SO}_{4}$, filtered, and concentrated under reduced pressure. The residue was purified by flash column chromatography on silica gel with EtOAc/petroleum ether $(1: 6)$ as the eluent to give pentacyclic $7(12.5 \mathrm{mg}, 50 \%)$ as a white foam. $7:[\alpha]_{\mathrm{D}}^{25}=-144.5\left(c=0.5\right.$ in $\left.\mathrm{CHCl}_{3}\right)$; IR (neat): $v\left(\mathrm{~cm}^{-1}\right)$ 2950, 1735, 1602, 1495, 1448, 1379, 1312, 1267, 1245, 1210, 1104, 1012, 975, 738; ${ }^{1} \mathrm{H}$ NMR (400 MHz, $\left.\mathrm{CDCl}_{3}\right): \delta 7.38(\mathrm{~d}, J=7.6 \mathrm{~Hz}, 1 \mathrm{H}), 7.04(\mathrm{t}, J=$ $7.6 \mathrm{~Hz}, 1 \mathrm{H}), 6.50$ (t, $J=7.6 \mathrm{~Hz}, 1 \mathrm{H}), 6.30(\mathrm{~d}, J=7.6 \mathrm{~Hz}, 1 \mathrm{H}), 5.60$ (q, $J=6.8 \mathrm{~Hz}$, 1H), $4.11(\mathrm{~d}, J=3.2 \mathrm{~Hz}, 1 \mathrm{H}), 3.90(\mathrm{~s}, 3 \mathrm{H}), 3.82(\mathrm{~d}, J=17.2 \mathrm{~Hz}, 1 \mathrm{H}), 3.45$ (s, 3H), $3.21(\mathrm{~d}, J=16.4 \mathrm{~Hz}, 1 \mathrm{H}), 2.99-3.12(\mathrm{~m} 1 \mathrm{H}), 3.01$ (s, 3H), 2.91 (dd, $J=12.0,8.4 \mathrm{~Hz}$, 1H), $2.49(\mathrm{dd}, J=20.0,4.8 \mathrm{~Hz}, 1 \mathrm{H}), 2.40(\mathrm{dd}, J=20.0,2.0 \mathrm{~Hz}, 1 \mathrm{H}), 2.30(\mathrm{td}, J=13.2$, $8.0 \mathrm{~Hz}, 1 \mathrm{H}), 2.13(\mathrm{dd}, J=13.2,8.0 \mathrm{~Hz}, 1 \mathrm{H}), 1.84(\mathrm{dd}, J=6.8,2.0 \mathrm{~Hz}, 3 \mathrm{H}) \mathrm{ppm} ;{ }^{13} \mathrm{C}$ NMR (101 MHz, $\left.\mathrm{CDCl}_{3}\right): \delta 207.4,170.3,169.7,148.8,136.7,133.1,128.1,126.9$, $122.7,116.3,104.1,97.0,65.3,63.3,57.6,54.8,52.7,52.6,45.7,41.5,37.1,29.1$, 14.8 ppm; ESI-MS: m/z $411.2\left(\mathrm{M}^{+}+\mathrm{H}\right)$; HRMS calcd for $\mathrm{C}_{23} \mathrm{H}_{27} \mathrm{~N}_{2} \mathrm{O}_{5}[\mathrm{M}+\mathrm{H}]$ : 411.1914, found 411.1911.
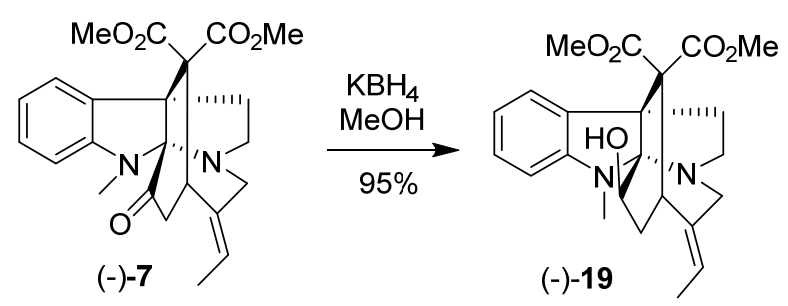

Compound 19. To a solution of pentacyclic 7 (15 mg, $0.037 \mathrm{mmol})$ in $\mathrm{MeOH}(10 \mathrm{~mL})$ was added $\mathrm{KBH}_{4}(40 \mathrm{mg}, 0.74 \mathrm{mmol})$ in 3 portions at room temperature. The mixture was stirred at room temperature for $2 \mathrm{~h}$. The reaction was then quenched with saturated aqueous $\mathrm{NaHCO}_{3}(10 \mathrm{~mL})$. The resulting mixture was extracted with $\mathrm{CH}_{2} \mathrm{Cl}_{2}(3 \times 10 \mathrm{~mL})$. The combined organic layers were washed with brine $(2 \times 30$ $\mathrm{mL}$ ), dried over anhydrous $\mathrm{Na}_{2} \mathrm{SO}_{4}$, filtered, and concentrated under reduced pressure. The residue was purified by flash column chromatography on silica gel with 
EtOAc/petroleum ether (1:2) as the eluent to give alcohol $19(14.3 \mathrm{mg}, 95 \%)$ as a white foam. 19: $[\alpha]_{\mathrm{D}}^{25}=-154.1\left(c=0.2\right.$ in $\left.\mathrm{CHCl}_{3}\right)$; IR (neat): $v\left(\mathrm{~cm}^{-1}\right) 3500,3358$, 2963, 2921, 2851, 1758, 1727, 1660, 1602, 1494, 1469, 1380, 1260, 1212, 1126, 1081, 1060, 845, 742; ${ }^{1} \mathrm{H}$ NMR (400 MHz, $\left.\mathrm{CDCl}_{3}\right): \delta 7.39(\mathrm{~d}, J=7.6 \mathrm{~Hz}, 1 \mathrm{H}), 7.06(\mathrm{t}, J=$ $7.6 \mathrm{~Hz}, 1 \mathrm{H}), 6.49$ (t, $J=7.6 \mathrm{~Hz}, 1 \mathrm{H}), 6.27$ (d, $J=7.6 \mathrm{~Hz}, 1 \mathrm{H}), 5.46$ (q, $J=6.8 \mathrm{~Hz}$, 1H), 4.34-4.44 (m, 1H), 3.95 (d, $J=12.4 \mathrm{~Hz}, 1 \mathrm{H}), 3.84$ (s, 3H), 3.78 (d, J=4.0 Hz, 1H), 3.73 (s, 3H), 3.71 (d, $J=16.4 \mathrm{~Hz}, 1 \mathrm{H}), 3.19$ (td, $J=11.2,8.8 \mathrm{~Hz}, 1 \mathrm{H}), 3.07$ (d, $J$ $=16.4 \mathrm{~Hz}, 1 \mathrm{H}), 2.80(\mathrm{~s}, 3 \mathrm{H}), 2.71(\mathrm{ddd}, J=11.6,8.0,1.6 \mathrm{~Hz}, 1 \mathrm{H}), 2.25(\mathrm{ddd}, J=15.6$, 10.4, 1.6 Hz, 1H), 1.93-2.08 (m, 2H), $1.83(\mathrm{dd}, J=6.8,2.0 \mathrm{~Hz}, 3 \mathrm{H}), 1.69-1.76$ (m,

1H) ppm; ${ }^{13} \mathrm{C}$ NMR $\left(101 \mathrm{MHz}, \mathrm{CDCl}_{3}\right): \delta 172.8,170.1,150.4,137.4,133.9,127.8$, $125.0,123.7,115.4,103.5,97.5,65.9,63.9,59.6,57.4,53.6,53.5,52.1,46.7,38.7$, 35.4, 27.1, $15.1 \mathrm{ppm}$; ESI-MS: m/z $413.1\left(\mathrm{M}^{+}+\mathrm{H}\right)$; HRMS calcd for $\mathrm{C}_{23} \mathrm{H}_{29} \mathrm{~N}_{2} \mathrm{O}_{5}$ $[\mathrm{M}+\mathrm{H}]:$ 413.2071, found 413.2066.
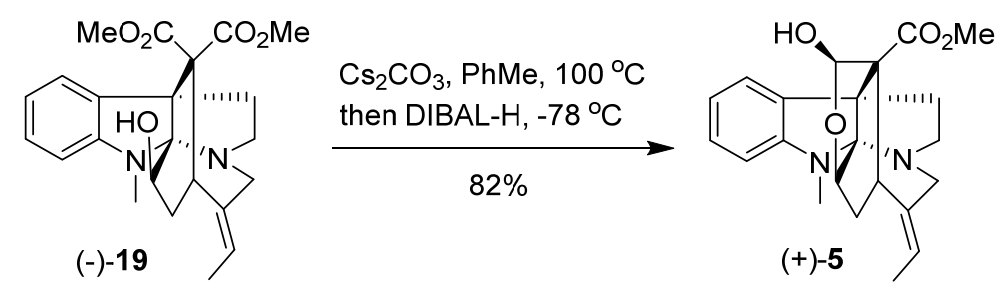

Corymine (5). To a solution of alcohol $19(5 \mathrm{mg}, 0.012 \mathrm{mmol})$ in toluene $(2 \mathrm{~mL})$ was added added $\mathrm{Cs}_{2} \mathrm{CO}_{3}(19.6 \mathrm{mg}, 0.06 \mathrm{mmol})$ at room temperature under argon atmosphere. The solution was stirred at $100{ }^{\circ} \mathrm{C}$ for $1 \mathrm{~h}$ and then cooled down to $-78^{\circ} \mathrm{C}$, DIBAL-H (1.0 M solution in hexane, $\left.33 \mu \mathrm{L}, 0.033 \mathrm{mmol}\right)$ was directly added in 3 portions. The mixture was stirred at $-78^{\circ} \mathrm{C}$ for another $0.5 \mathrm{~h}$. The reaction was then quenched with aqueous sodium potassium tartrate $(5 \mathrm{~mL})$. The resulting mixture was extracted with EtOAc $(3 \times 5 \mathrm{~mL})$. The combined organic layers were washed with brine $(2 \times 15 \mathrm{~mL})$, dried over anhydrous $\mathrm{Na}_{2} \mathrm{SO}_{4}$, filtered, and concentrated under reduced pressure. The residue was purified by flash column chromatography on silica gel with $\mathrm{MeOH} / \mathrm{CH}_{2} \mathrm{Cl}_{2}(1: 40)$ as the eluent to give corymine 5 (3.8 $\left.\mathrm{mg}, 82 \%\right)$ as a white foam. 5: $[\alpha]_{\mathrm{D}}^{18}=+18.5\left(c=0.1\right.$ in $\left.\mathrm{CHCl}_{3}\right)$; IR (neat): $v\left(\mathrm{~cm}^{-1}\right) 3261,3047$, 
2951, 1726, 1675, 1601, 1493, 1453, 1380, 1309, 1262, 1227, 1155, 1121, 1059, 1010, 980, 816, 738; ${ }^{1} \mathrm{H}$ NMR (400 MHz, DMSO-d $\left.)_{6}\right): \delta .15(\mathrm{~d}, J=7.2 \mathrm{~Hz}, 1 \mathrm{H}), 6.87$ (t, $J=$ $7.2 \mathrm{~Hz}, 1 \mathrm{H}), 6.71(\mathrm{~d}, J=4.8 \mathrm{~Hz}, 1 \mathrm{H}), 6.35$ (t, $J=7.2 \mathrm{~Hz}, 1 \mathrm{H}), 6.15$ (d, $J=7.2 \mathrm{~Hz}$, 1H), 5.34 (q, $J=6.8 \mathrm{~Hz}, 1 \mathrm{H}), 4.93(\mathrm{~d}, J=4.8 \mathrm{~Hz}, 1 \mathrm{H}), 4.29$ (d, $J=4.0 \mathrm{~Hz}, 1 \mathrm{H}), 3.91$ $(\mathrm{d}, J=16.0 \mathrm{~Hz}, 1 \mathrm{H}), 3.76(\mathrm{~s}, 3 \mathrm{H}), 3.68(\mathrm{~d}, J=7.6 \mathrm{~Hz}, 1 \mathrm{H}), 2.95(\mathrm{~d}, J=16.0 \mathrm{~Hz}, 1 \mathrm{H})$, $2.92(\mathrm{td}, J=12.0,7.2 \mathrm{~Hz}, 1 \mathrm{H}), 2.77(\mathrm{dd}, J=12.0,8.0 \mathrm{~Hz}, 1 \mathrm{H}), 2.61-2.70(\mathrm{~m}, 4 \mathrm{H})$, $2.58(\mathrm{td}, J=12.8,7.6 \mathrm{~Hz}, 1 \mathrm{H}), 1.81(\mathrm{dd}, J=14.0,4.0 \mathrm{~Hz}, 1 \mathrm{H}), 1.65(\mathrm{dd}, J=6.8,0.8$ $\mathrm{Hz}, 3 \mathrm{H}), 1.39(\mathrm{dd}, J=12.8,6.4 \mathrm{~Hz}, 1 \mathrm{H}) \mathrm{ppm} ;{ }^{13} \mathrm{C}$ NMR $\left(101 \mathrm{MHz}, \mathrm{DMSO}-d_{6}\right): \delta$ 172.6, 152.1, 141.1, 134.0, 127.3, 124.0, 120.3, 114.7, 102.9, 96.3, 94.3, 68.5, 61.9, 57.4, 56.8, 51.8, 51.0, 39.0, 32.8, 29.2, 27.1, 13.8 ppm; ESI-MS: m/z 383.1 $\left(\mathrm{M}^{+}+\mathrm{H}\right)$; HRMS calcd for $\mathrm{C}_{22} \mathrm{H}_{27} \mathrm{~N}_{2} \mathrm{O}_{4}[\mathrm{M}+\mathrm{H}]: 383.1965$, found 383.1962 .

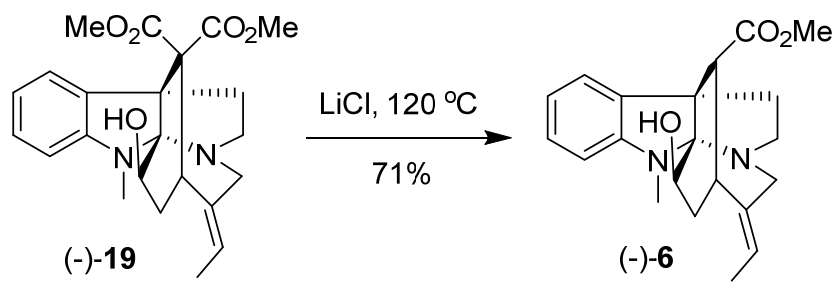

Deformylcorymine (6). To a solution of alcohol $19(8.5 \mathrm{mg}, 0.021 \mathrm{mmol})$ in DMF (2 $\mathrm{mL})$ was added $\mathrm{LiCl}(8.9 \mathrm{mg}, 0.21 \mathrm{mmol})$ and $\mathrm{H}_{2} \mathrm{O}(19 \mu \mathrm{L}, 1.05 \mathrm{mmol})$ at room temperature under argon atmosphere. The mixture was stirred at $120{ }^{\circ} \mathrm{C}$ for $5 \mathrm{~h}$. The reaction was then quenched with saturated aqueous $\mathrm{NaHCO}_{3}(20 \mathrm{~mL})$. The resulting mixture was extracted with $\mathrm{CH}_{2} \mathrm{Cl}_{2}(3 \times 10 \mathrm{~mL})$. The combined organic layers were washed with brine $(2 \times 30 \mathrm{~mL})$, dried over anhydrous $\mathrm{Na}_{2} \mathrm{SO}_{4}$, filtered, and concentrated under reduced pressure. The residue was purified by preparative TLC (silica gel, $\left.\mathrm{MeOH} / \mathrm{CH}_{2} \mathrm{Cl}_{2}, 1 / 20\right)$ to give deformylcorymine $6(5.2 \mathrm{mg}, 71 \%$ ) as a white solid. 6: $[\alpha]_{\mathrm{D}}^{25}=-26.3\left(c=0.1\right.$ in EtOH); IR (neat): $v\left(\mathrm{~cm}^{-1}\right) 3358,3193,2960$, 2921, 2852, 1736, 1660, 1630, 1604, 1491, 1469, 1378, 1261, 1176, 1022, 876, 801, 740; ${ }^{1} \mathrm{H}$ NMR (400 MHz, $\left.\mathrm{CDCl}_{3}\right): \delta 7.19(\mathrm{~d}, J=7.2 \mathrm{~Hz}, 1 \mathrm{H}), 7.02(\mathrm{td}, J=7.2,0.8 \mathrm{~Hz}$, 1H), $6.54(\mathrm{t}, J=7.2 \mathrm{~Hz}, 1 \mathrm{H}), 6.27(\mathrm{~d}, J=7.2 \mathrm{~Hz}, 1 \mathrm{H}), 5.39$ (q, $J=6.8 \mathrm{~Hz}, 1 \mathrm{H}), 4.45$ $(\mathrm{d}, J=8.0 \mathrm{~Hz}, 1 \mathrm{H}), 3.80(\mathrm{~s}, 3 \mathrm{H}), 3.72(\mathrm{~d}, J=5.2 \mathrm{~Hz}, 1 \mathrm{H}), 3.61(\mathrm{~d}, J=15.6 \mathrm{~Hz}, 1 \mathrm{H})$, 
$3.23(\mathrm{~s}, 1 \mathrm{H}), 3.12(\mathrm{td}, J=11.6,8.4 \mathrm{~Hz}, 1 \mathrm{H}), 2.98(\mathrm{~d}, J=15.6 \mathrm{~Hz}, 1 \mathrm{H}), 2.80(\mathrm{~s}, 3 \mathrm{H})$, $2.73(\mathrm{dd}, J=12.0,8.8 \mathrm{~Hz}, 1 \mathrm{H}), 2.61(\mathrm{ddd}, J=13.6,11.2,8.8 \mathrm{~Hz}, 1 \mathrm{H}), 2.22(\mathrm{dd}, J=$ 14.4, $9.2 \mathrm{~Hz}, 1 \mathrm{H}), 1.99$ (dd, $J=14.4,5.2 \mathrm{~Hz}, 1 \mathrm{H}), 1.91$ (dd, $J=13.6,8.0 \mathrm{~Hz}, 1 \mathrm{H}$ ), $1.80(\mathrm{br}, 1 \mathrm{H}), 1.58$ (dd, $J=6.8,1.6 \mathrm{~Hz}, 3 \mathrm{H}) \mathrm{ppm} ;{ }^{13} \mathrm{C} \mathrm{NMR}\left(101 \mathrm{MHz}, \mathrm{CDCl}_{3}\right): \delta$ $173.7,149.5,138.7,137.9,127.8,122.8,122.6,117.0,103.5,97.1,66.8,57.9,56.6$, 55.2, 51.6, 48.2, 43.1, 37.2, 35.0, 26.4, 13.5 ppm; ESI-MS: m/z 355.1 (M+H); HRMS calcd for $\mathrm{C}_{21} \mathrm{H}_{27} \mathrm{~N}_{2} \mathrm{O}_{3}[\mathrm{M}+\mathrm{H}]$ : 355.2016, found 355.2012.

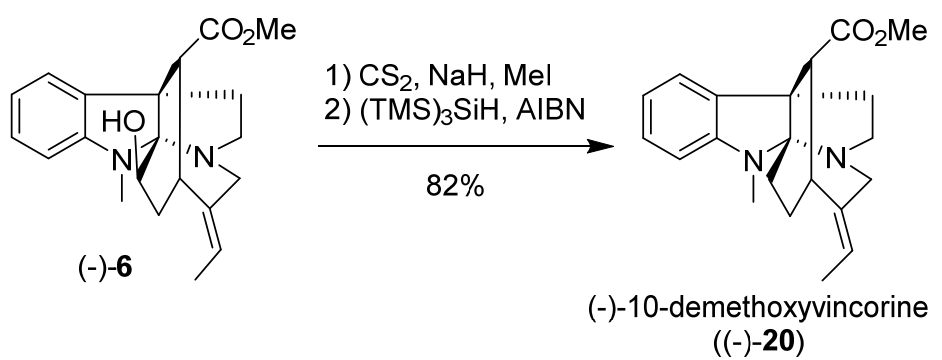

10-Demethoxyvincorine (20). To a solution of deformylcorymine 6 (5 mg, 0.014 mmol) in THF $(1 \mathrm{~mL})$ were added $\mathrm{CS}_{2}(9 \mu \mathrm{L}, 0.14 \mathrm{mmol})$ and $\mathrm{NaH}(5.6 \mathrm{mg}, 0.14$ mmol) at $0{ }^{\circ} \mathrm{C}$ under argon atmosphere. The solution was stirred at $0{ }^{\circ} \mathrm{C}$ for $0.5 \mathrm{~h}$, and MeI (9 $\mu \mathrm{L}, 0.14 \mathrm{mmol})$ was added. The mixture was allowed to warm to room temperature and was stirred for another $2 \mathrm{~h}$. The reaction was then quenched with saturated aqueous $\mathrm{NH}_{4} \mathrm{Cl}(5 \mathrm{~mL})$. The resulting mixture was extracted with $\mathrm{CH}_{2} \mathrm{Cl}_{2}$ (3 $\times 5 \mathrm{~mL})$. The combined organic layers were washed with brine $(2 \times 15 \mathrm{~mL})$, dried over anhydrous $\mathrm{Na}_{2} \mathrm{SO}_{4}$, filtered, and concentrated under reduced pressure. The residue was dissolved in benzene $(10 \mathrm{~mL})$. AIBN $(2.3 \mathrm{mg}, 0.014 \mathrm{mmol})$ and $(\mathrm{TMS})_{3} \mathrm{SiH}(44 \mu \mathrm{L}, 0.14 \mathrm{mmol})$ were added to the solution at room temperature under argon atmosphere. The mixture was stirred at $80{ }^{\circ} \mathrm{C}$ for $0.5 \mathrm{~h}$. The resulting mixture was cooled down to room temperature and concentrated under reduced pressure. The residue was purified by flash column chromatography on silica gel with EtOAc/petroleum ether (1:1) as the eluent to give 10-demethoxyvincorine 20 (3.9 $\mathrm{mg}$, 82\%) as a white film. 20: $[\alpha]_{\mathrm{D}}^{25}=-95.5\left(c=0.2\right.$ in $\left.\mathrm{CHCl}_{3}\right)$; IR (neat): $v\left(\mathrm{~cm}^{-1}\right) 3046$, 
2948, 2874, 2856, 1736, 1605, 1488, 1375, 1225, 1174, 804, 739; ${ }^{1} \mathrm{H}$ NMR (400 MHz, $\left.\mathrm{CDCl}_{3}\right): \delta 7.23(\mathrm{~d}, J=7.2 \mathrm{~Hz}, 1 \mathrm{H}), 7.06(\mathrm{td}, J=7.2,0.8 \mathrm{~Hz}, 1 \mathrm{H}), 6.58(\mathrm{t}, J=7.2 \mathrm{~Hz}$, 1H), $6.27(\mathrm{~d}, J=7.2 \mathrm{~Hz}, 1 \mathrm{H}), 5.39(\mathrm{q}, J=6.8 \mathrm{~Hz}, 1 \mathrm{H}), 3.75-3.84(\mathrm{~m}, 4 \mathrm{H}), 3.61(\mathrm{~d}, J$ $=3.6 \mathrm{~Hz}, 1 \mathrm{H}), 3.32(\mathrm{td}, J=11.2,9.2 \mathrm{~Hz}, 1 \mathrm{H}), 3.00(\mathrm{~d}, J=15.2 \mathrm{~Hz}, 1 \mathrm{H}), 2.83(\mathrm{~s}, 1 \mathrm{H})$, 2.69-2.77 (m, 1H), $2.62(\mathrm{~s}, 3 \mathrm{H}), 2.44-2.54(\mathrm{~m}, 1 \mathrm{H}), 2.26-2.36(\mathrm{~m}, 1 \mathrm{H}), 1.97(\mathrm{dd}, J=$ 14.0, 7.6 Hz, 1H), 1.64-1.81 (m, 3H), 1.59 (dd, $J=6.8,1.6 \mathrm{~Hz}, 3 \mathrm{H}) \mathrm{ppm} ;{ }^{13} \mathrm{C} \mathrm{NMR}$ (101 MHz, $\left.\mathrm{CDCl}_{3}\right): \delta 174.0,149.3,139.4,137.3,128.0,123.7,122.4,117.0,105.4$, 97.6, 58.5, 57.2, 55.3, 51.8, 50.9, 41.4, 35.0, 27.4, 26.5, 21.1, 13.7 ppm; ESI-MS: m/z $339.1\left(\mathrm{M}^{+}+\mathrm{H}\right)$; HRMS calcd for $\mathrm{C}_{21} \mathrm{H}_{27} \mathrm{~N}_{2} \mathrm{O}_{2}[\mathrm{M}+\mathrm{H}]$ : 339.2067, found 339.2067.
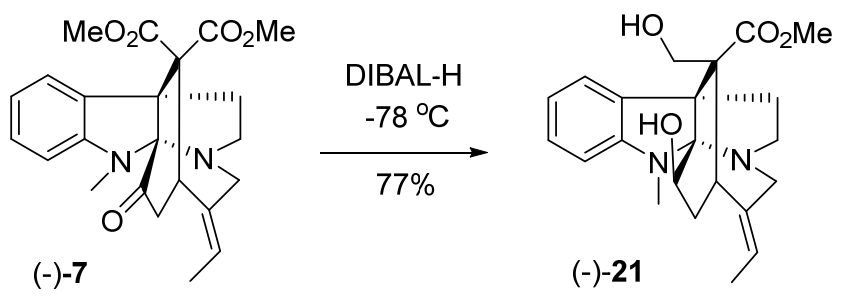

Compound 21. To a solution of pentacyclic $7(6 \mathrm{mg}, 0.015 \mathrm{mmol})$ in toluene $(2.5 \mathrm{~mL})$ was added DIBAL-H (1.0 M solution in hexane, $0.15 \mathrm{~mL}, 0.15 \mathrm{mmol})$ in 3 portions at $-78^{\circ} \mathrm{C}$ under argon. After stirred at $-78^{\circ} \mathrm{C}$ for $0.5 \mathrm{~h}$, the reaction was quenched with saturated aqueous sodium potassium tartrate $(5 \mathrm{~mL})$ and the mixture was then stirred for $2 \mathrm{~h}$. The resulting mixture was extracted with EtOAc $(3 \times 5 \mathrm{~mL})$. The combined organic layers were washed with brine $(2 \times 15 \mathrm{~mL})$, dried over anhydrous $\mathrm{Na}_{2} \mathrm{SO}_{4}$, filtered, and concentrated under reduced pressure. The residue was purified by flash column chromatography on silica gel with $\mathrm{MeOH} / \mathrm{CH}_{2} \mathrm{Cl}_{2}(1: 20)$ as the eluent to give dihydrocorymine $21(4.3 \mathrm{mg}, 77 \%)$ as a white solid. 21 : $[\alpha]_{\mathrm{D}}^{25}=-137.7(c=0.25$ in $\mathrm{MeOH})$; IR (KBr): $v\left(\mathrm{~cm}^{-1}\right)$ 3367, 3045, 2958, 2858, 1727, 1660, 1600, 1490, 1380, 1261, 911, 802, 737; ${ }^{1} \mathrm{H}$ NMR (400 MHz, $\left.\mathrm{CDCl}_{3}\right): \delta 7.52$ (d, J= $\left.7.2 \mathrm{~Hz}, 1 \mathrm{H}\right), 7.06$ (t, $J=7.2 \mathrm{~Hz}, 1 \mathrm{H}), 6.58(\mathrm{t}, J=7.2 \mathrm{~Hz}, 1 \mathrm{H}), 6.34(\mathrm{~d}, J=7.2 \mathrm{~Hz}, 1 \mathrm{H}), 5.35$ (q, $J=6.8 \mathrm{~Hz}$, 1H), 4.39 (d, $J=9.6 \mathrm{~Hz}, 1 \mathrm{H}), 4.24(\mathrm{~d}, J=12.0 \mathrm{~Hz}, 1 \mathrm{H}), 4.14(\mathrm{~d}, J=12.0 \mathrm{~Hz}, 1 \mathrm{H})$, 3.81-3.86 (m, 4H), $3.74(\mathrm{~d}, J=16.0 \mathrm{~Hz}, 1 \mathrm{H}), 3.01(\mathrm{~d}, J=16.0 \mathrm{~Hz}, 1 \mathrm{H}), 2.75-2.86(\mathrm{~m}$, 
4H), $2.70(\mathrm{dd}, J=12.4,8.4 \mathrm{~Hz}, 1 \mathrm{H}), 2.42(\mathrm{br}, 1 \mathrm{H}), 2.21-2.36(\mathrm{~m}, 2 \mathrm{H}), 2.04$ (ddd, $J=$ $15.6,10.0,1.6 \mathrm{~Hz}, 1 \mathrm{H}), 1.93(\mathrm{dd}, J=13.6,7.6 \mathrm{~Hz}, 1 \mathrm{H}), 1.70(\mathrm{dd}, J=6.8,1.6 \mathrm{~Hz}, 3 \mathrm{H})$ ppm; ${ }^{13} \mathrm{C} \mathrm{NMR}\left(101 \mathrm{MHz}, \mathrm{CDCl}_{3}\right): \delta 175.6,150.4,139.9,132.6,128.2,126.0,122.7$, $117.1,104.7,95.4,66.2,65.2,60.4,58.4,56.0,53.5,51.9,48.3,35.6,31.0,26.8,14.3$ ppm; ESI-MS: m/z $385.2\left(\mathrm{M}^{+}+\mathrm{H}\right)$; HRMS calcd for $\mathrm{C}_{22} \mathrm{H}_{29} \mathrm{~N}_{2} \mathrm{O}_{4}[\mathrm{M}+\mathrm{H}]: 385.2122$, found 385.2121 .
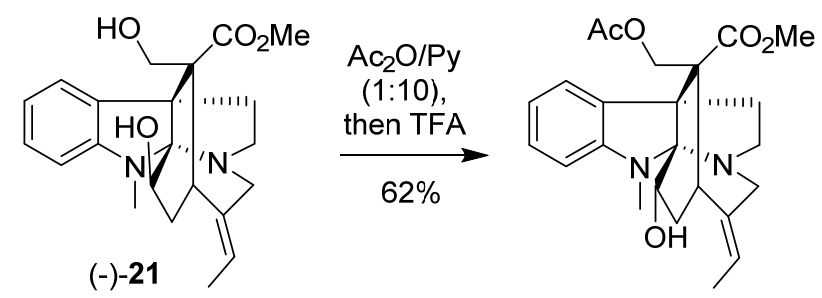

(-)-3-epi-dihydrocorymine 17-acetate ((-)-22)

3-epi-Dihydrocorymine 17-acetate (22). To a solution of dihydrocorymine 21 (4.0 $\mathrm{mg}, 0.11 \mathrm{mmol})$ in pyridine $(1 \mathrm{~mL})$ was added $\mathrm{Ac}_{2} \mathrm{O}(0.1 \mathrm{~mL})$ at room temperature under argon atmosphere. After stirred at room temperature for $24 \mathrm{~h}$, the resulting mixture was concentrated under reduced pressure. The residue was dissolved in TFA $(1 \mathrm{~mL})$. The mixture was stirred at room temperature for another $48 \mathrm{~h}$. The reaction was then quenched with saturated aqueous $\mathrm{NaHCO}_{3}(20 \mathrm{~mL})$. The resulting mixture was extracted with $\mathrm{CH}_{2} \mathrm{Cl}_{2}(3 \times 20 \mathrm{~mL})$. The combined organic layers were washed with brine $(2 \times 60 \mathrm{~mL})$, dried over anhydrous $\mathrm{Na}_{2} \mathrm{SO}_{4}$, filtered, and concentrated under reduced pressure. The residue was purified by preparative TLC (silica gel, $\left.\mathrm{MeOH} / \mathrm{CH}_{2} \mathrm{Cl}_{2}, 1 / 20\right)$ to give 3-epi-dihydrocorymine 17 -acetate $22(2.8 \mathrm{mg}, 62 \%)$ as a white film. $[\alpha]_{\mathrm{D}}^{25}=-59.5\left(c=0.1\right.$ in $\left.\mathrm{CHCl}_{3}\right)$; IR $(\mathrm{KBr}): v\left(\mathrm{~cm}^{-1}\right) 3447,3048,2958$, 2924, 2855, 1744, 1670, 1600, 1494, 1377, 1249, 1122, 1085, 984, 736; ${ }^{1} \mathrm{H}$ NMR $\left(400 \mathrm{MHz}, \mathrm{CDCl}_{3}\right): \delta 7.64(\mathrm{~d}, J=7.6 \mathrm{~Hz}, 1 \mathrm{H}), 7.06(\mathrm{td}, J=7.6,1.2 \mathrm{~Hz}, 1 \mathrm{H}), 6.54(\mathrm{td}$, $J=7.6,0.8 \mathrm{~Hz}, 1 \mathrm{H}), 6.24(\mathrm{~d}, J=7.6 \mathrm{~Hz}, 1 \mathrm{H}), 5.41(\mathrm{q}, J=6.8 \mathrm{~Hz}, 1 \mathrm{H}), 4.86(\mathrm{~d}, J=$ $12.0 \mathrm{~Hz}, 1 \mathrm{H}), 4.35$ (dd, $J=10.8,5.2 \mathrm{~Hz}, 1 \mathrm{H}), 4.17$ (d, $J=16.4 \mathrm{~Hz}, 1 \mathrm{H}), 3.78$ (s, 3H), 3.69-3.75 (m, 2H), $3.07(\mathrm{~d}, J=16.4 \mathrm{~Hz}, 1 \mathrm{H}), 3.01(\mathrm{td}, J=12.0,8.0 \mathrm{~Hz}, 1 \mathrm{H}), 2.90(\mathrm{~s}$, 
3H), $2.76(\mathrm{dd}, J=12.0,8.4 \mathrm{~Hz}, 1 \mathrm{H}), 2.42-2.53(\mathrm{~m}, 1 \mathrm{H}), 2.23(\mathrm{br}, 1 \mathrm{H}), 2.07-2.15(\mathrm{~m}$, 1H), $2.06(\mathrm{~s}, 3 \mathrm{H}), 1.95(\mathrm{dd}, J=13.6,7.6 \mathrm{~Hz}, 1 \mathrm{H}), 1.77(\mathrm{dd}, J=6.8,2.0 \mathrm{~Hz}, 3 \mathrm{H}), 1.54$ (ddd, $\left.J=15.6,5.6,1.2 \mathrm{~Hz}, 1 \mathrm{H}) \mathrm{ppm} ;{ }^{13} \mathrm{C} \mathrm{NMR} \mathrm{(101} \mathrm{MHz,} \mathrm{CDCl}_{3}\right): \delta 173.4,170.3$, $149.7,139.4,130.0,128.5,126.5,123.1,116.0,104.8,96.4,70.5,67.1,61.9,57.7$, 54.7, 54.0, 51.9, 47.0, 35.8, 33.8, 28.6, 20.9, 14.7 ppm; ESI-MS: m/z $427.2\left(\mathrm{M}^{+}+\mathrm{H}\right)$; HRMS calcd for $\mathrm{C}_{24} \mathrm{H}_{31} \mathrm{~N}_{2} \mathrm{O}_{5}[\mathrm{M}+\mathrm{H}]$ : 427.2227, found 427.2227.
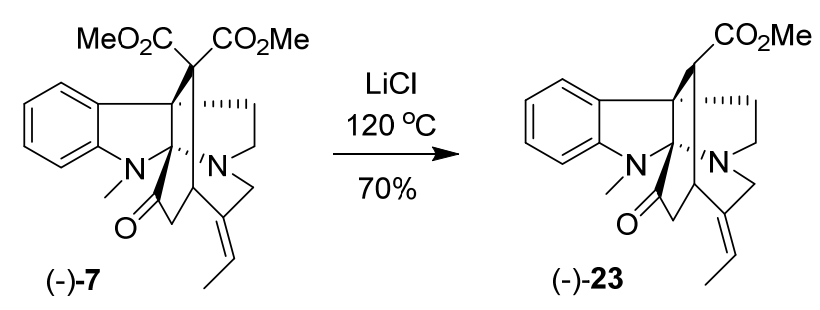

Compound 23. To a solution of pentacyclic 7 (6.0 mg, $0.015 \mathrm{mmol})$ in DMF $(1.5 \mathrm{~mL})$ were added $\mathrm{LiCl}(6.4 \mathrm{mg}, 0.15 \mathrm{mmol})$ and $\mathrm{H}_{2} \mathrm{O}(14 \mu \mathrm{L}, 0.75 \mathrm{mmol})$ at room temperature under argon atmosphere. The mixture was stirred at $120^{\circ} \mathrm{C}$ for $5 \mathrm{~h}$. The reaction was then quenched with saturated aqueous $\mathrm{NaHCO}_{3}(5 \mathrm{~mL})$. The resulting mixture was extracted with $\mathrm{CH}_{2} \mathrm{Cl}_{2}(3 \times 5 \mathrm{~mL})$. The combined organic layers were washed with brine $(2 \times 15 \mathrm{~mL})$, dried over anhydrous $\mathrm{Na}_{2} \mathrm{SO}_{4}$, filtered, and concentrated under reduced pressure. The residue was purified by flash column chromatography on silica gel with EtOAc/petroleum ether $(1: 6)$ as the eluent to give $23(3.6 \mathrm{mg}, 70 \%)$ as a white film. 23: $[\alpha]_{\mathrm{D}}^{25}=-75.7\left(c=0.15\right.$ in $\left.\mathrm{CHCl}_{3}\right)$; IR $(\mathrm{KBr})$ : $v$ $\left(\mathrm{cm}^{-1}\right)$ 3048, 2949, 2876, 1724, 1605, 1491, 1468, 1450, 1377, 1309, 1215, 1129, 1008, 977, 890, 740; ${ }^{1} \mathrm{H} \mathrm{NMR}\left(400 \mathrm{MHz}, \mathrm{CDCl}_{3}\right): \delta 7.18(\mathrm{dd}, J=7.6,0.8 \mathrm{~Hz}, 1 \mathrm{H})$, $7.05(\mathrm{td}, J=7.6,0.8 \mathrm{~Hz}, 1 \mathrm{H}), 6.51(\mathrm{td}, J=7.6,0.8 \mathrm{~Hz}, 1 \mathrm{H}), 6.29(\mathrm{~d}, J=7.6 \mathrm{~Hz}, 1 \mathrm{H})$, $5.56(\mathrm{q}, J=6.8 \mathrm{~Hz}, 1 \mathrm{H}), 3.93(\mathrm{~s}, 1 \mathrm{H}), 3.84(\mathrm{~s}, 3 \mathrm{H}), 3.69$ (d, $J=15.6 \mathrm{~Hz}, 1 \mathrm{H}), 3.26$ (dd, $J=20.4,10.8 \mathrm{~Hz}, 1 \mathrm{H}), 3.17(\mathrm{~d}, J=15.6 \mathrm{~Hz}, 1 \mathrm{H}), 2.98(\mathrm{~s}, 1 \mathrm{H}), 2.96(\mathrm{~s}, 3 \mathrm{H})$, 2.86-2.94 (m 1H), 2.59-2.67 (m 1H), $2.55(\mathrm{dd}, J=19.6,1.2 \mathrm{~Hz}, 1 \mathrm{H}), 2.42(\mathrm{dd}, J=$ 19.6, 4.4 Hz, 1H), 2.08 (ddd, $J=13.6,8.8,1.6 \mathrm{~Hz}, 1 \mathrm{H}), 1.68(\mathrm{dd}, J=6.8,2.0 \mathrm{~Hz}, 3 \mathrm{H})$ ppm; ${ }^{13} \mathrm{C} \mathrm{NMR}\left(101 \mathrm{MHz}, \mathrm{CDCl}_{3}\right): \delta 211.1,172.5,148.8,136.5,135.0,128.4,125.0$, 
122.7, 116.2, 104.4, 100.1, 61.0, 58.1, 56.3, 51.9, 51.4, 45.4, 43.3, 34.8, 28.8, 13.7 ppm; ESI-MS: m/z 353.1 $\left(\mathrm{M}^{+}+\mathrm{H}\right)$; HRMS calcd for $\mathrm{C}_{21} \mathrm{H}_{25} \mathrm{~N}_{2} \mathrm{O}_{3}[\mathrm{M}+\mathrm{H}]$ : 353.1860, found 353.1856 .

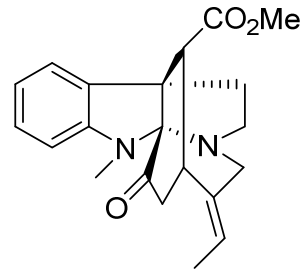

$(-)-23$

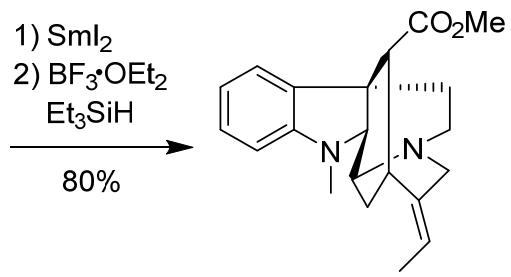

$(-)-2(S)$-cathafoline ((-)-24)

2(S)-cathafoline (24). To a solution of compound $23(5.0 \mathrm{mg}, 0.014 \mathrm{mmol})$ in THF/MeOH (1.5 mL/0.3 mL) was added $\mathrm{SmI}_{2}(0.1 \mathrm{M}$ solution in THF, $1.4 \mathrm{~mL}, 0.14$ mmol) at room temperature under argon atmosphere. The mixture was stirred at room temperature for $0.5 \mathrm{~h}$. The reaction was then quenched with saturated aqueous $\mathrm{NaHCO}_{3}(5 \mathrm{~mL})$. The resulting mixture was extracted with $\mathrm{CH}_{2} \mathrm{Cl}_{2}(3 \times 5 \mathrm{~mL})$. The combined organic layers were washed with brine $(2 \times 15 \mathrm{~mL})$, dried over anhydrous $\mathrm{Na}_{2} \mathrm{SO}_{4}$, filtered, and concentrated under reduced pressure. The residue was dissolved in $\mathrm{CH}_{2} \mathrm{Cl}_{2}(1 \mathrm{~mL}) . \mathrm{Et}_{3} \mathrm{SiH}(0.2 \mathrm{~mL})$ and $\mathrm{BF}_{3} \cdot \mathrm{OEt}_{2}(0.1 \mathrm{~mL})$ were added to the solution at room temperature under argon atmosphere. The mixture was stirred at room temperature for $36 \mathrm{~h}$. The reaction was then quenched with saturated aqueous $\mathrm{NaHCO}_{3}(10 \mathrm{~mL})$. The resulting mixture was extracted with $\mathrm{CH}_{2} \mathrm{Cl}_{2}(3 \times 10 \mathrm{~mL})$. The combined organic layers were washed with brine $(2 \times 30 \mathrm{~mL})$, dried over anhydrous $\mathrm{Na}_{2} \mathrm{SO}_{4}$, filtered, and concentrated under reduced pressure. The residue was purified by flash column chromatography on silica gel with $\mathrm{MeOH} / \mathrm{CH}_{2} \mathrm{Cl}_{2}(1: 20)$ as the eluent to give $2(S)$-cathafoline $24(3.8 \mathrm{mg}, 80 \%)$ as a colorless oil. $24:[\alpha]_{\mathrm{D}}^{25}=-129.8(\mathrm{c}=$ 0.1 in $\mathrm{CHCl}_{3}$ ); IR (neat): $v\left(\mathrm{~cm}^{-1}\right)$ 2955, 2922, 2851, 1735, 1607, 1464, 1318, 1260 , 1165, 1122, 1038, 911, 740; ${ }^{1} \mathrm{H}$ NMR (400 MHz, $\left.\mathrm{CDCl}_{3}\right): \delta 7.07-7.15$ (m, 2H), $6.75(\mathrm{t}$, $J=7.6 \mathrm{~Hz}, 1 \mathrm{H}), 6.62(\mathrm{~d}, J=7.6 \mathrm{~Hz}, 1 \mathrm{H}), 5.38(\mathrm{q}, J=6.8 \mathrm{~Hz}, 1 \mathrm{H}), 3.89-3.99(\mathrm{~m}, 2 \mathrm{H})$, $3.53(\mathrm{~s}, 3 \mathrm{H}), 3.43(\mathrm{td}, J=12.8,6.4 \mathrm{~Hz}, 1 \mathrm{H}), 3.38(\mathrm{br}, 1 \mathrm{H}), 3.25-3.35(\mathrm{~m}, 2 \mathrm{H}), 2.95(\mathrm{~d}$, 
$J=16.4 \mathrm{~Hz}, 1 \mathrm{H}), 2.71(\mathrm{~d}, J=4.4 \mathrm{~Hz}, 1 \mathrm{H}), 2.68(\mathrm{~s}, 3 \mathrm{H}), 2.55(\mathrm{dd}, J=14.0,7.2 \mathrm{~Hz}$, 1H), $2.10(\mathrm{dd}, J=13.6,2.4 \mathrm{~Hz}, 1 \mathrm{H}), 2.04(\mathrm{dd}, J=14.4,6.0 \mathrm{~Hz}, 1 \mathrm{H}), 1.94-2.02$ (m, $1 \mathrm{H}), 1.44(\mathrm{dd}, J=6.8,2.0 \mathrm{~Hz}, 3 \mathrm{H}) \mathrm{ppm} ;{ }^{13} \mathrm{C} \mathrm{NMR}\left(101 \mathrm{MHz}, \mathrm{CDCl}_{3}\right): \delta 172.9,152.7$, 139.1, 138.1, 127.4, 123.3, 119.4, 118.9, 109.0, 70.4, 56.4, 50.9, 49.7, 48.5, 46.5, 43.0, 33.9, 32.6, 29.1, 25.4, 12.8 ppm; ESI-MS: m/z $339.1\left(\mathrm{M}^{+}+\mathrm{H}\right)$; HRMS calcd for $\mathrm{C}_{21} \mathrm{H}_{27} \mathrm{~N}_{2} \mathrm{O}_{2}[\mathrm{M}+\mathrm{H}]:$ 339.2067, found 339.2066. 


\section{5. ${ }^{1} \mathrm{H}$ and ${ }^{13} \mathrm{C}$ NMR spectra of all compounds.}

${ }^{1} \mathrm{H}$ NMR Spectrum of $10\left(400 \mathrm{MHz}, \mathrm{CDCl}_{3}\right)$
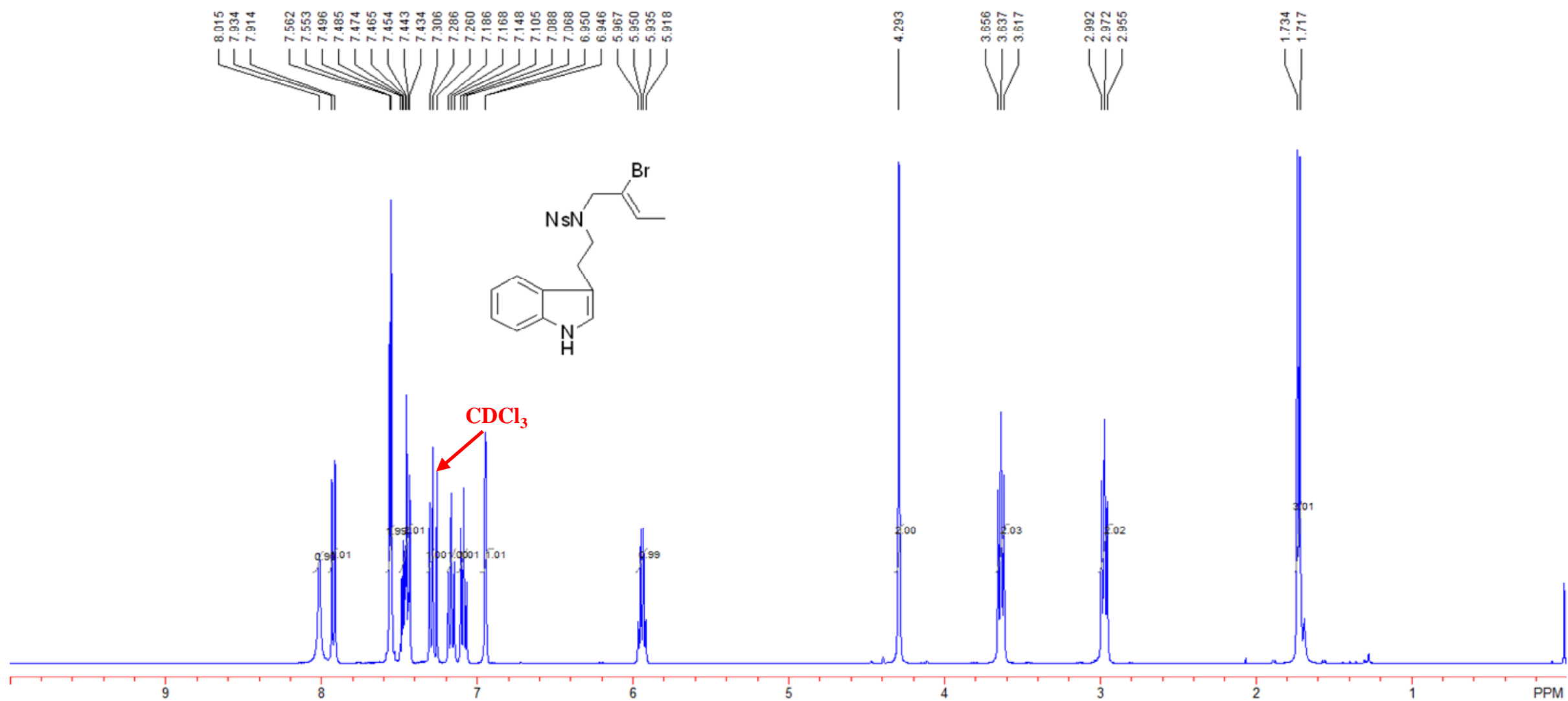
${ }^{13} \mathrm{C}$ NMR Spectrum of $\mathbf{1 0}\left(101 \mathrm{MHz}, \mathrm{CDCl}_{3}\right)$
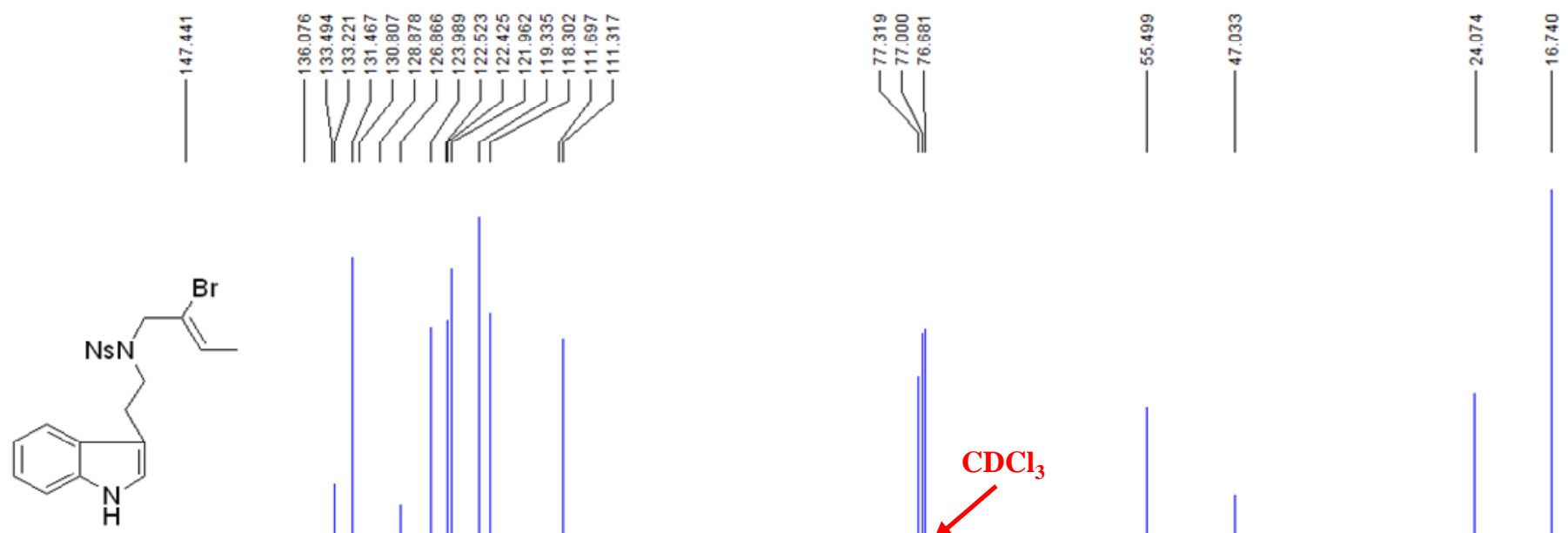

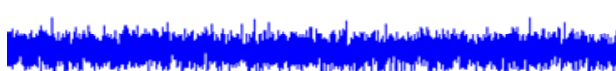

150
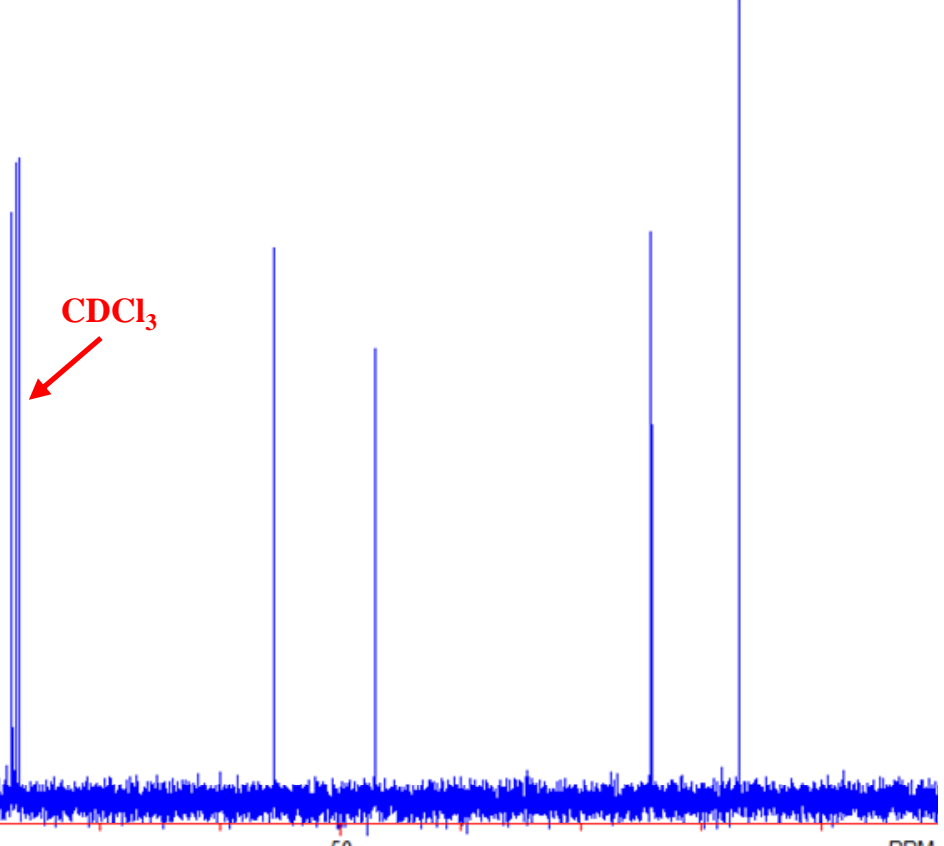
${ }^{1} \mathrm{H}$ NMR Spectrum of $11\left(400 \mathrm{MHz}, \mathrm{CDCl}_{3}\right)$
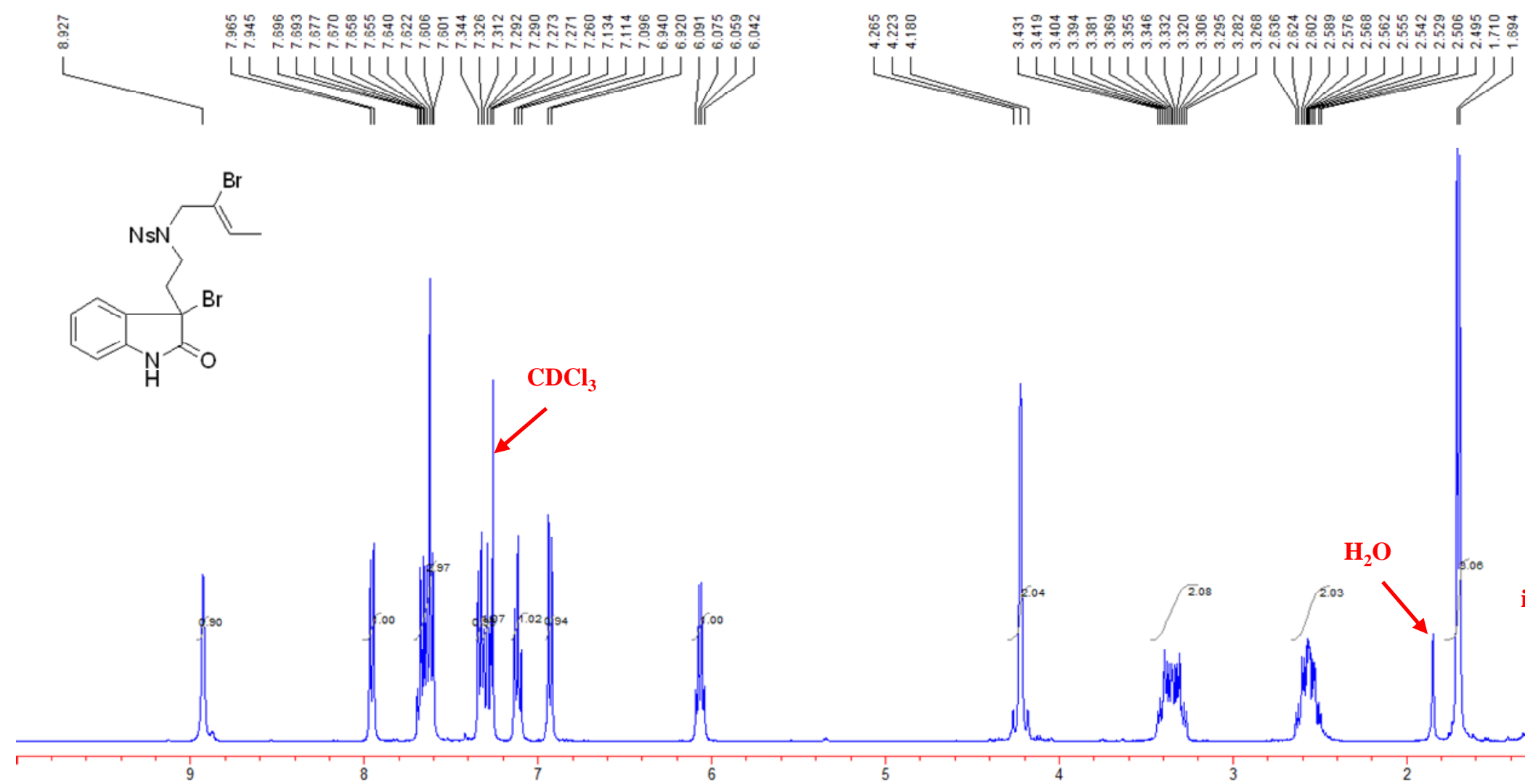

$\mathrm{CDCl}_{3}$ 
${ }^{13} \mathrm{C}$ NMR Spectrum of 11 (101 MHz, $\left.\mathrm{CDCl}_{3}\right)$
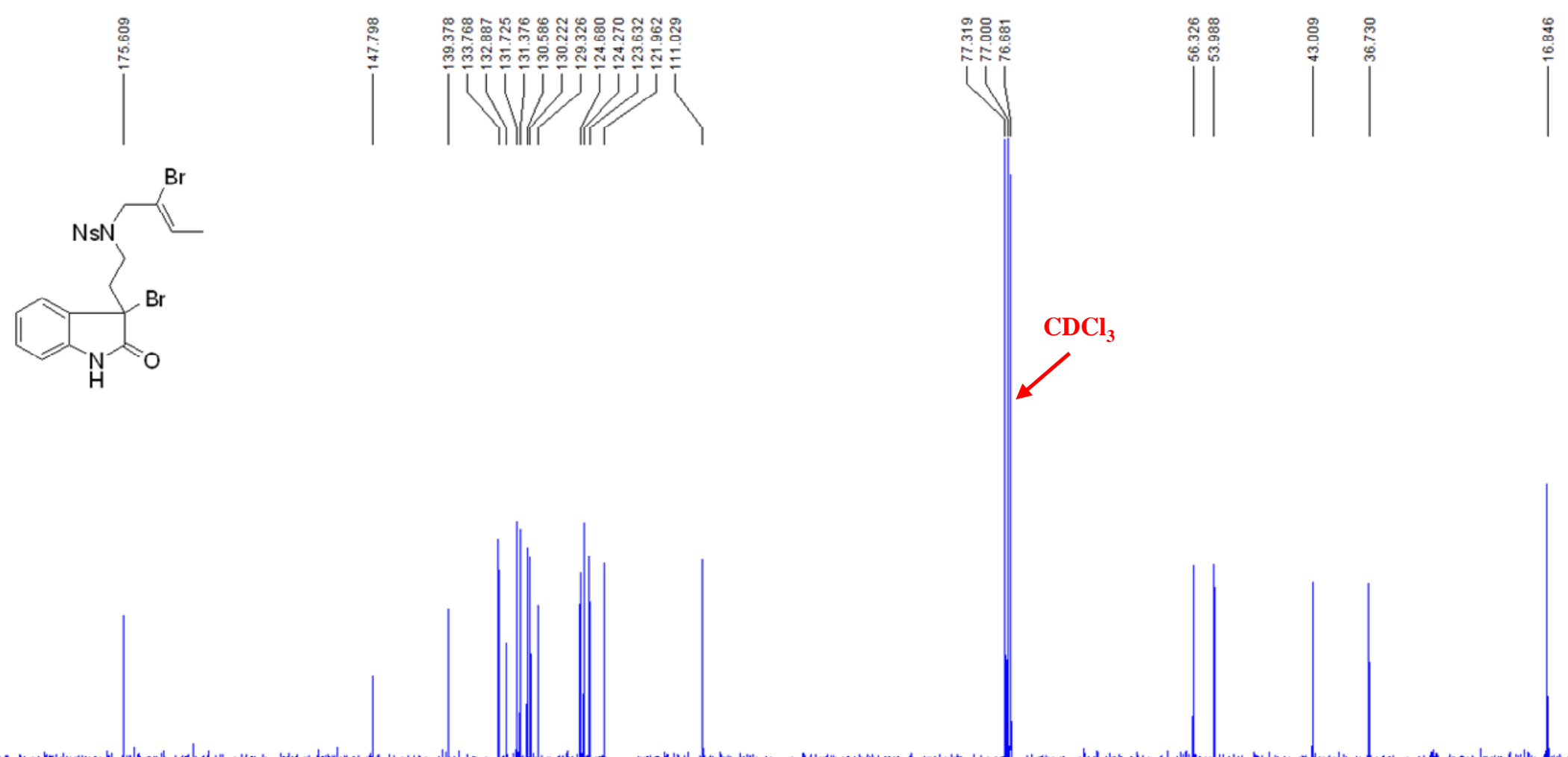

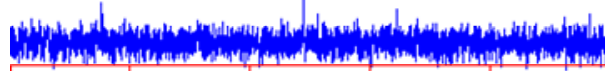


${ }^{1} \mathrm{H}$ NMR Spectrum of $12\left(400 \mathrm{MHz}, \mathrm{CDCl}_{3}\right)$
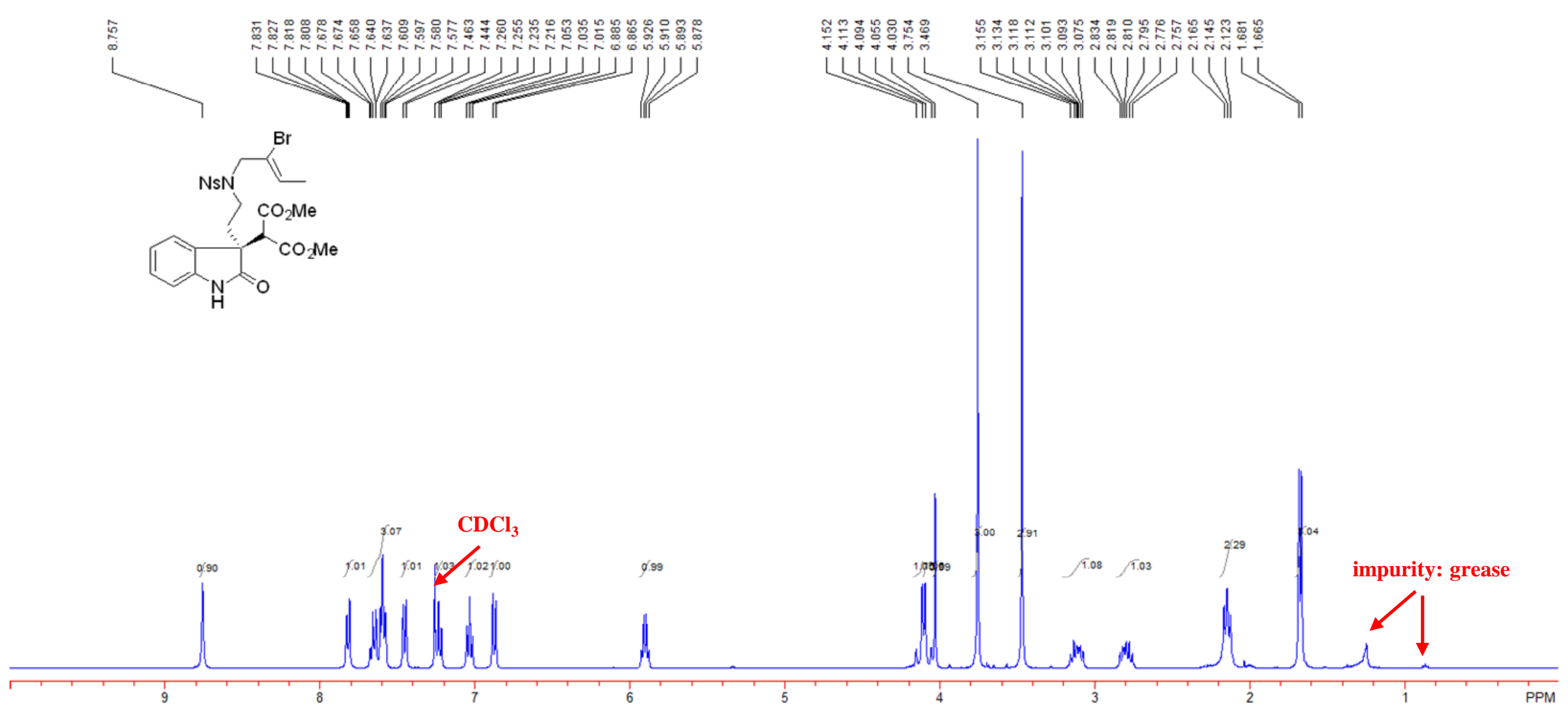
${ }^{13} \mathrm{C}$ NMR Spectrum of $12\left(101 \mathrm{MHz}, \mathrm{CDCl}_{3}\right)$

$$
\text { i }
$$
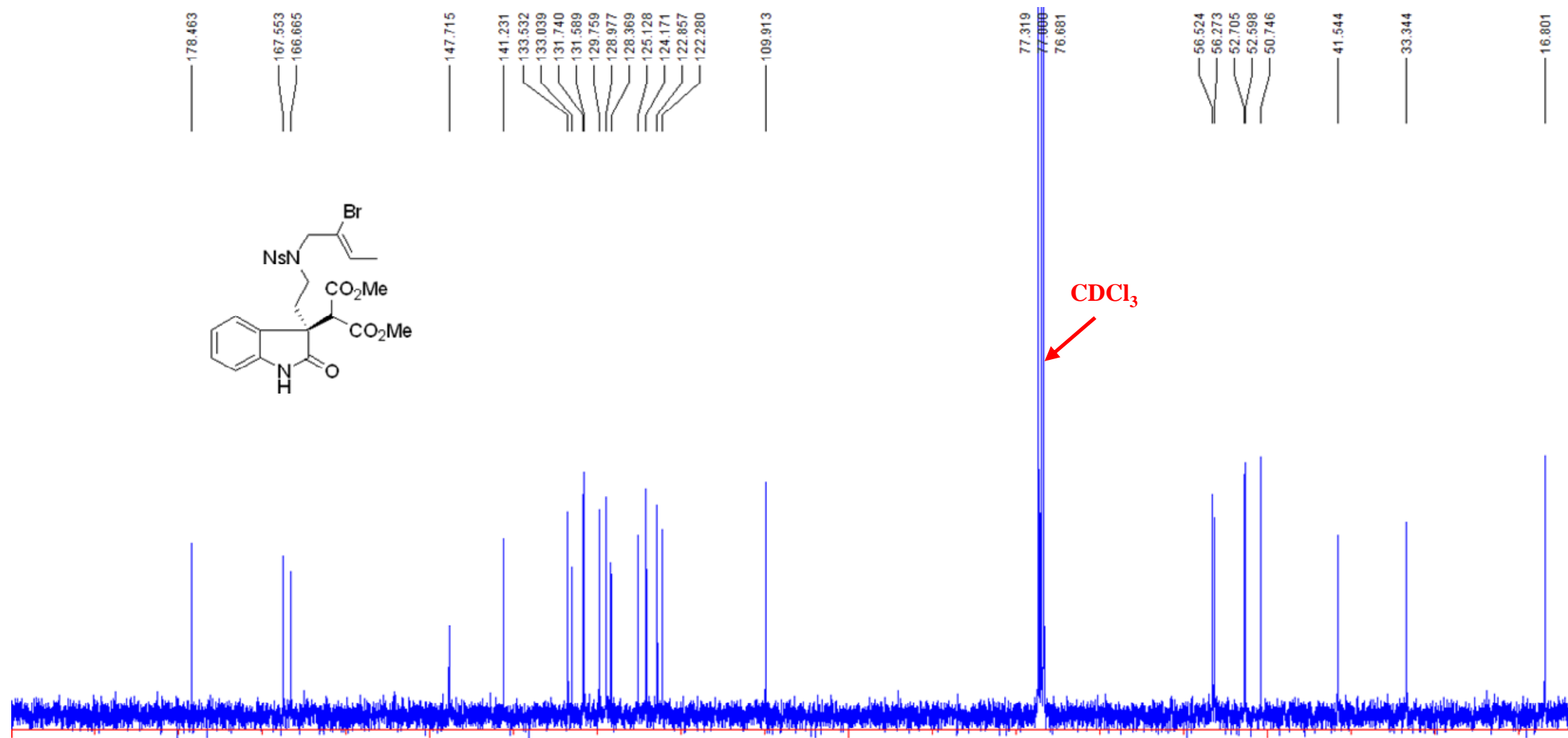
${ }^{1} \mathrm{H}$ NMR Spectrum of 15 (400 MHz, $\mathrm{CDCl}_{3}$ )
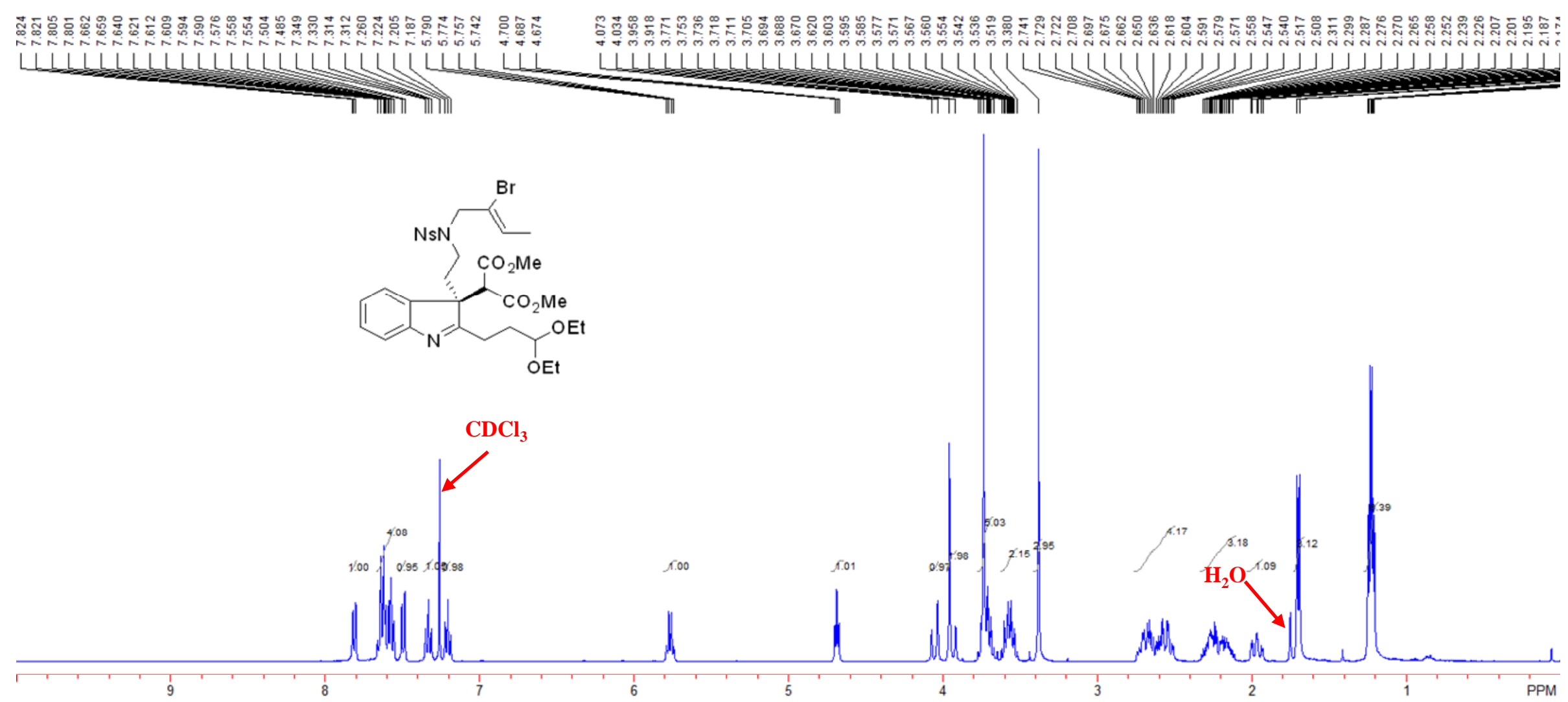
${ }^{13} \mathrm{C}$ NMR Spectrum of $15\left(101 \mathrm{MHz}, \mathrm{CDCl}_{3}\right)$
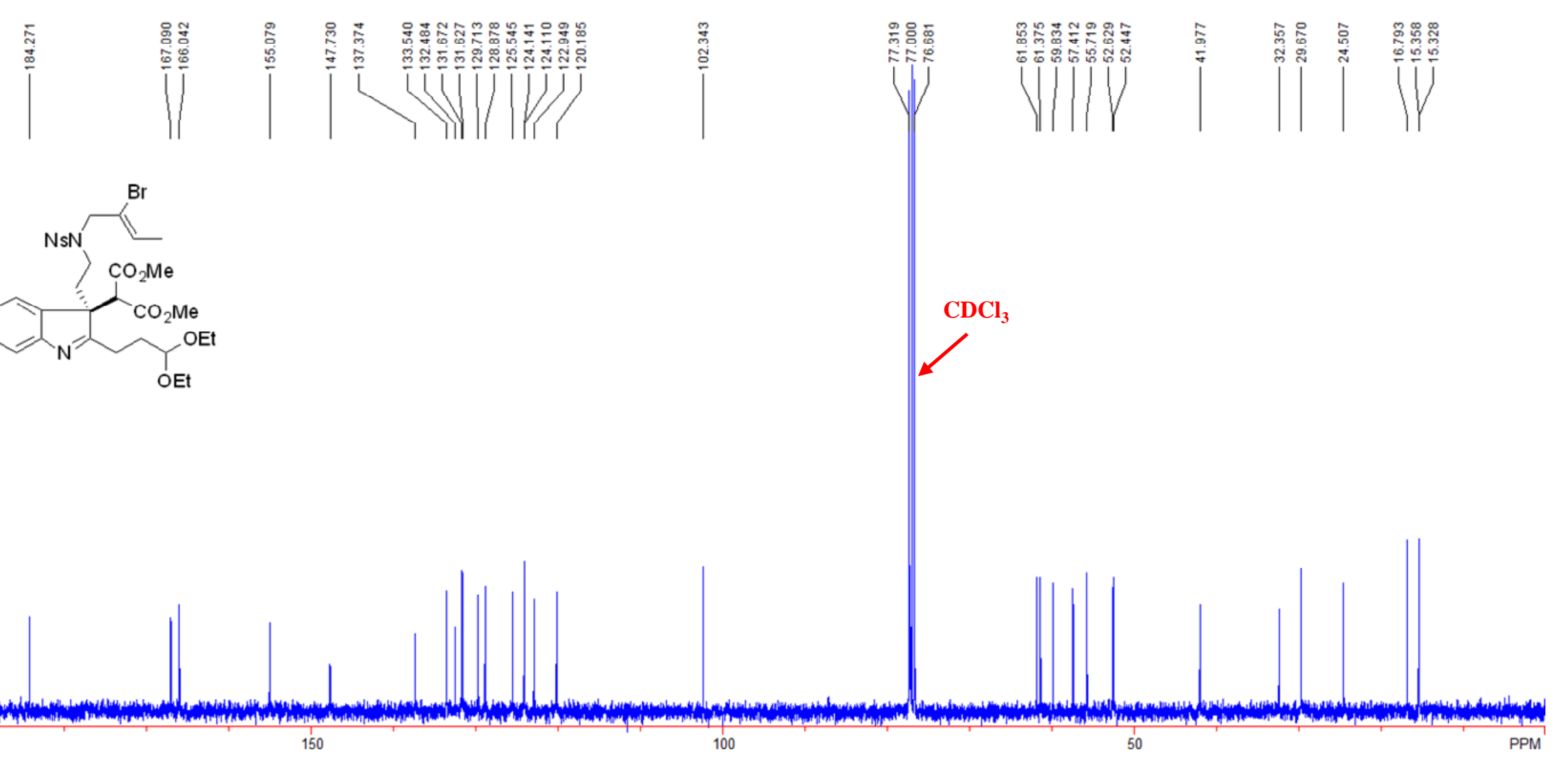
${ }^{1} \mathrm{H}$ NMR Spectrum of $16\left(400 \mathrm{MHz}, \mathrm{CDCl}_{3}\right)$

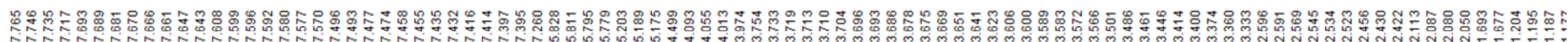
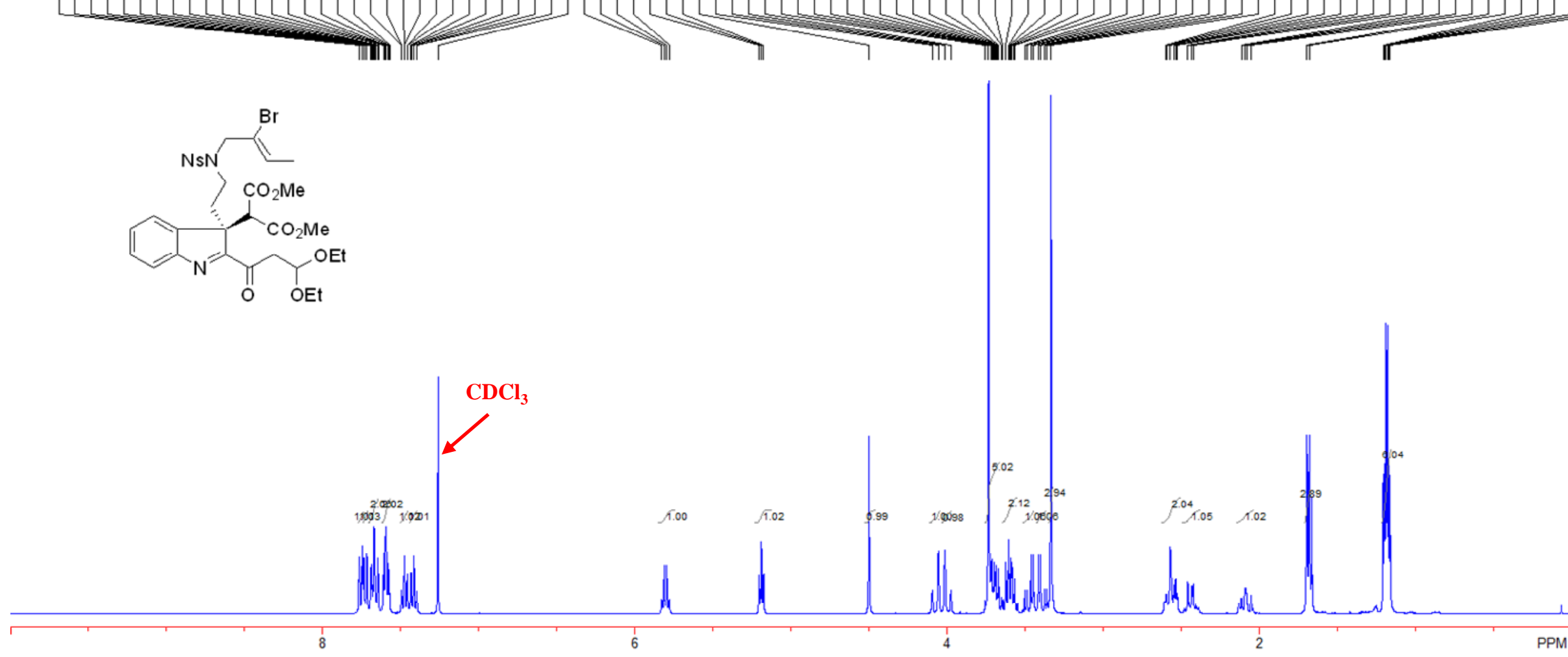
${ }^{13} \mathrm{C}$ NMR Spectrum of $16\left(101 \mathrm{MHz}, \mathrm{CDCl}_{3}\right)$
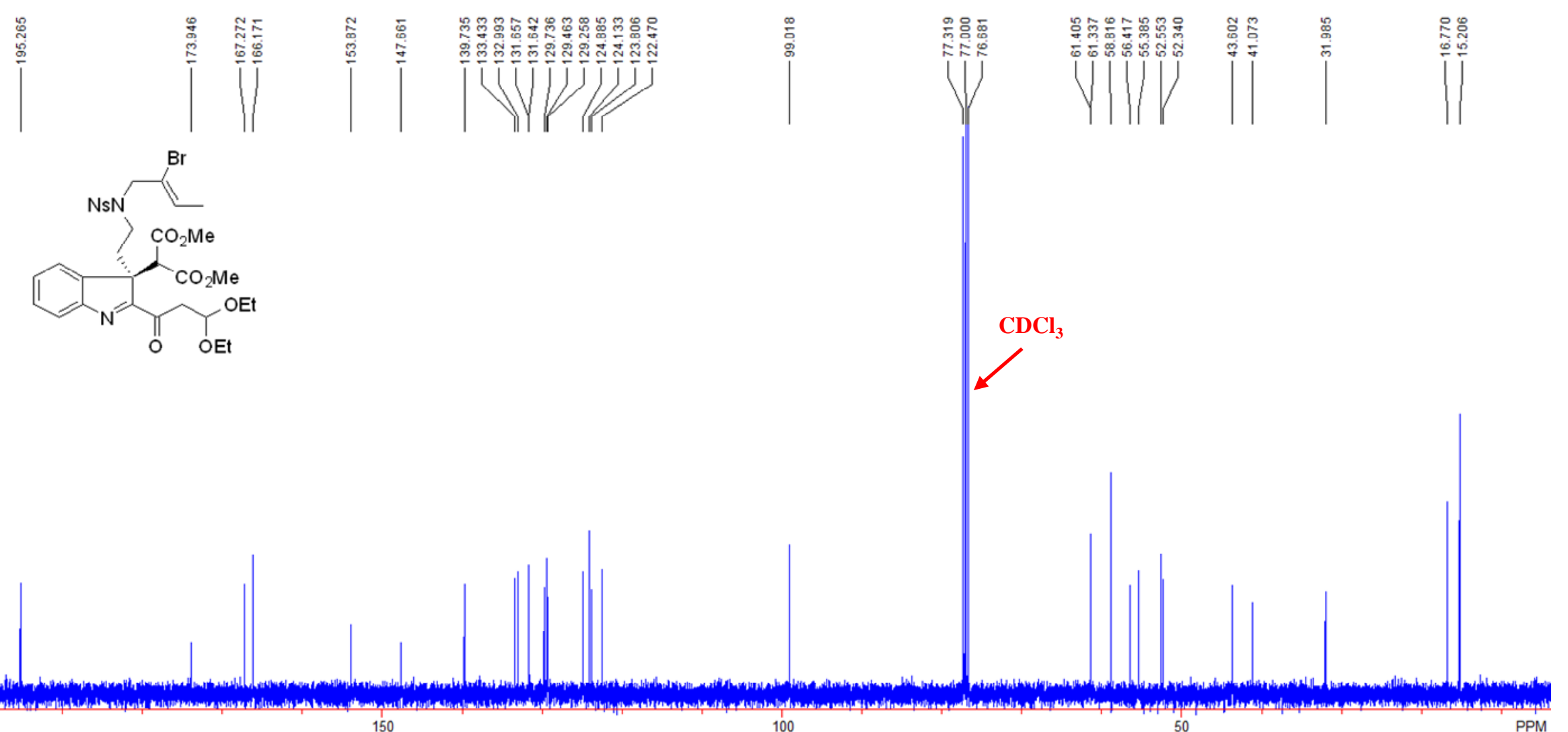

200

150

100

50 
${ }^{1} \mathrm{H}$ NMR Spectrum of $18\left(400 \mathrm{MHz}, \mathrm{CDCl}_{3}\right)$

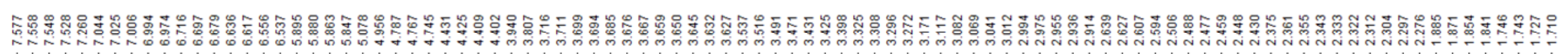

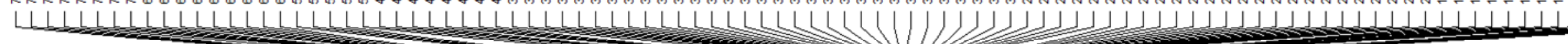
(1)
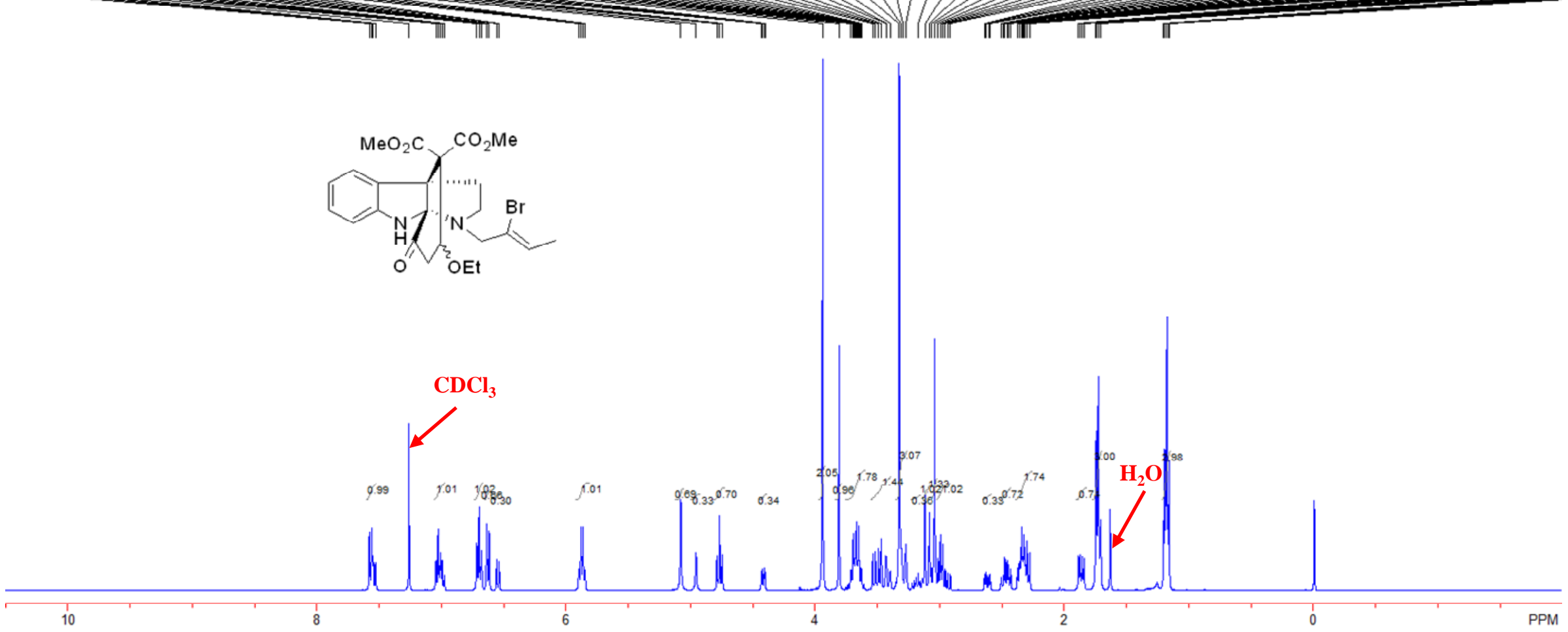
${ }^{13} \mathrm{C}$ NMR Spectrum of $18\left(101 \mathrm{MHz}, \mathrm{CDCl}_{3}\right)$
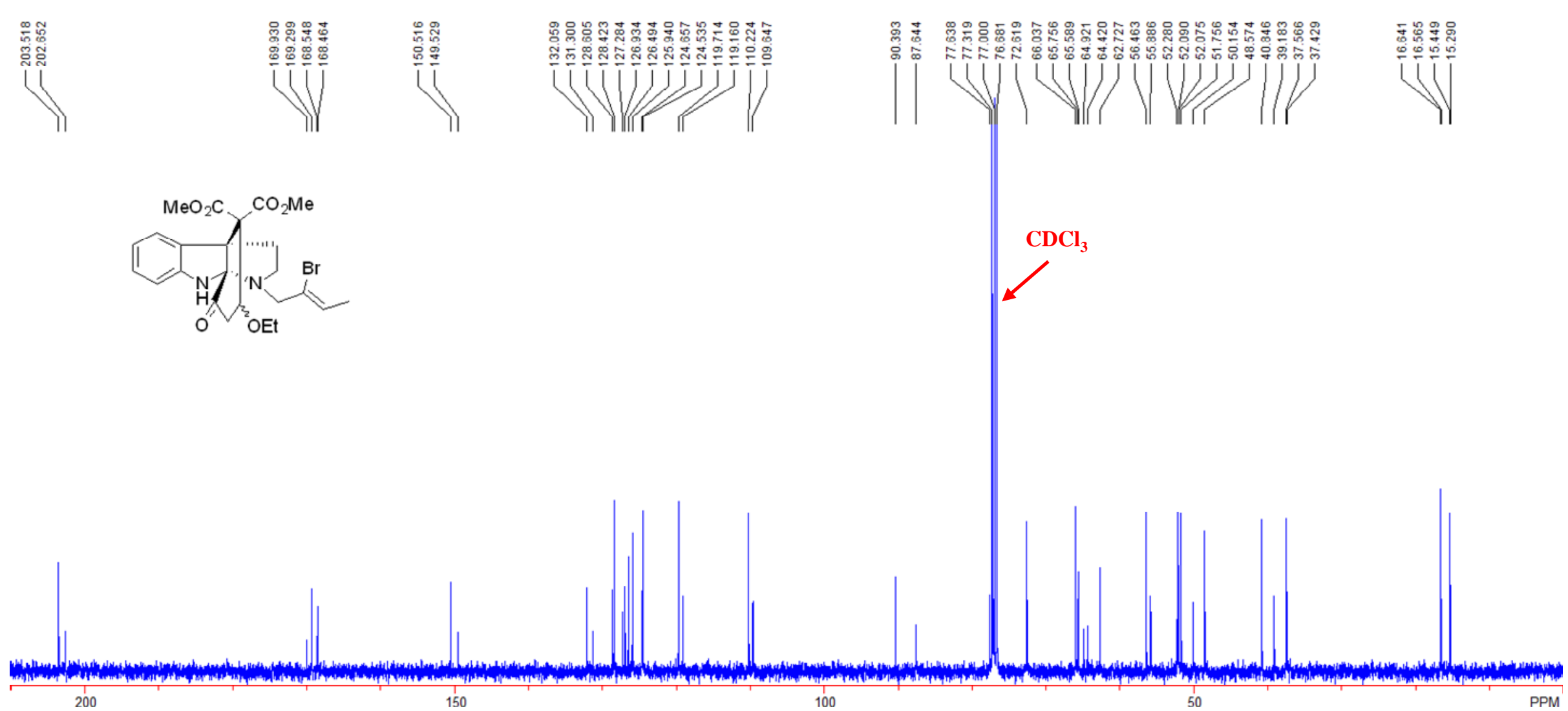
${ }^{1} \mathrm{H}$ NMR Spectrum of $8\left(400 \mathrm{MHz}, \mathrm{CDCl}_{3}\right)$

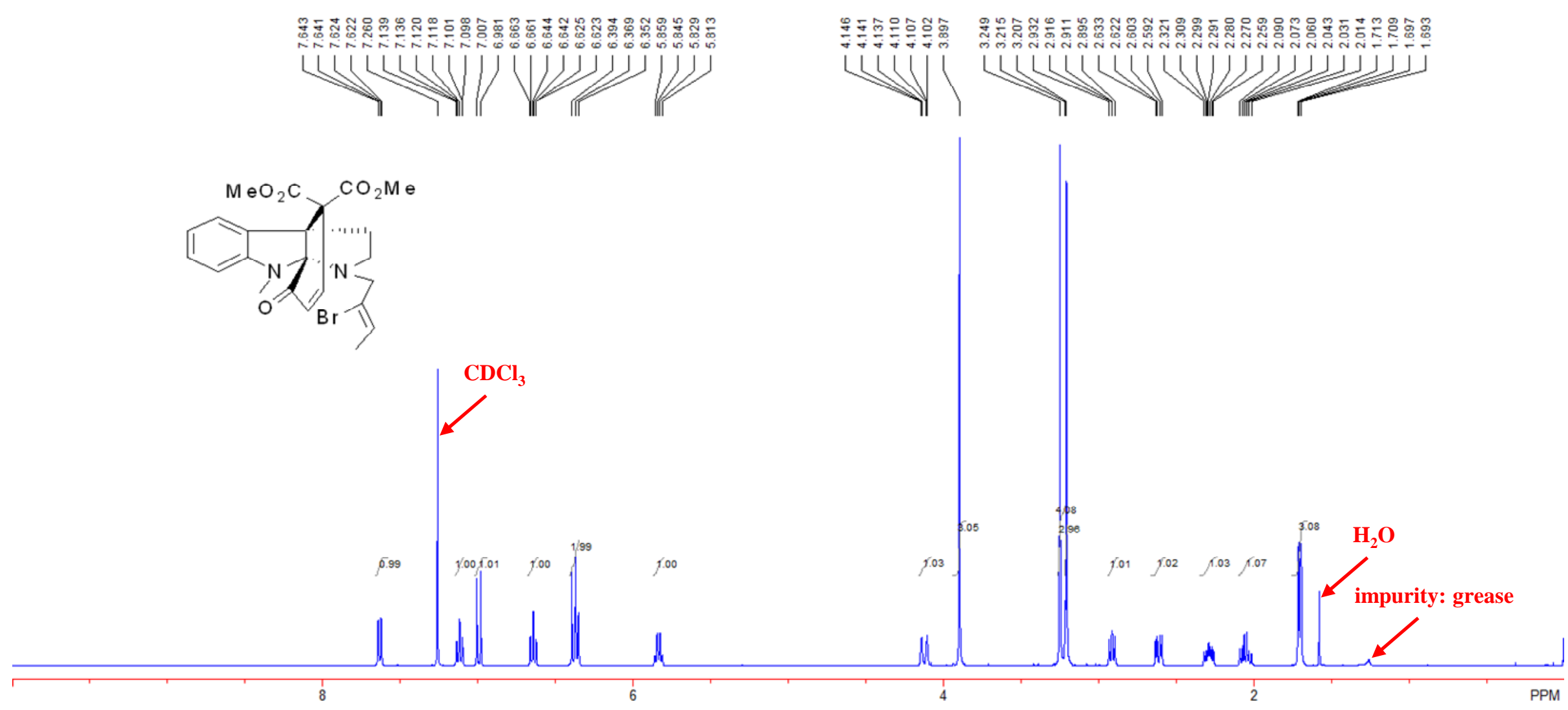


${ }^{13} \mathrm{C}$ NMR Spectrum of $8\left(101 \mathrm{MHz}, \mathrm{CDCl}_{3}\right)$
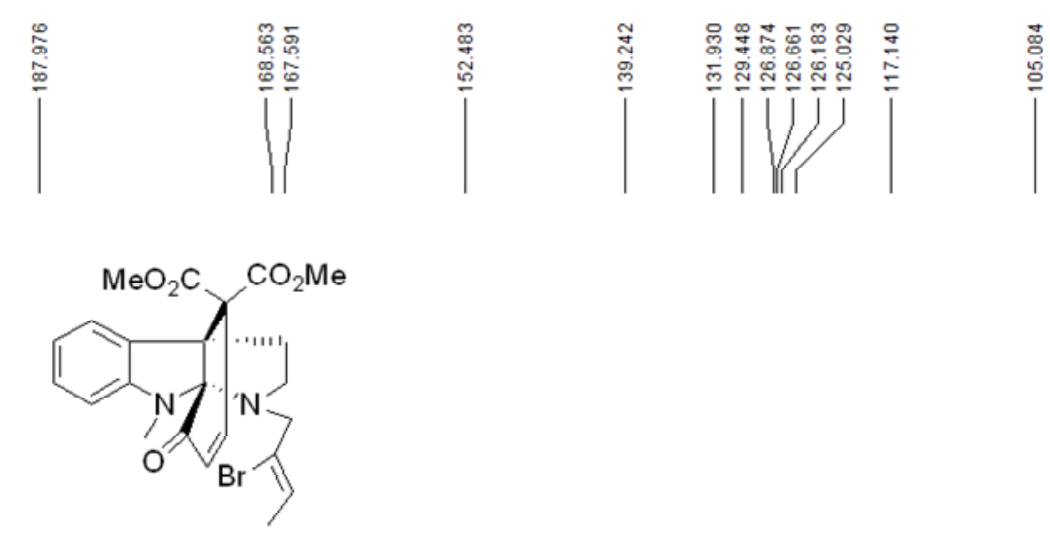

|

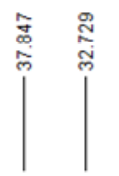

$\prod_{\substack{\infty \\ 6}}^{\substack{6 \\ \infty}}$

$\mathrm{CDCl}_{3}$

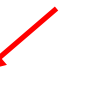


${ }^{1} \mathrm{H}$ NMR Spectrum of 7 (400 MHz, $\mathrm{CDCl}_{3}$ )
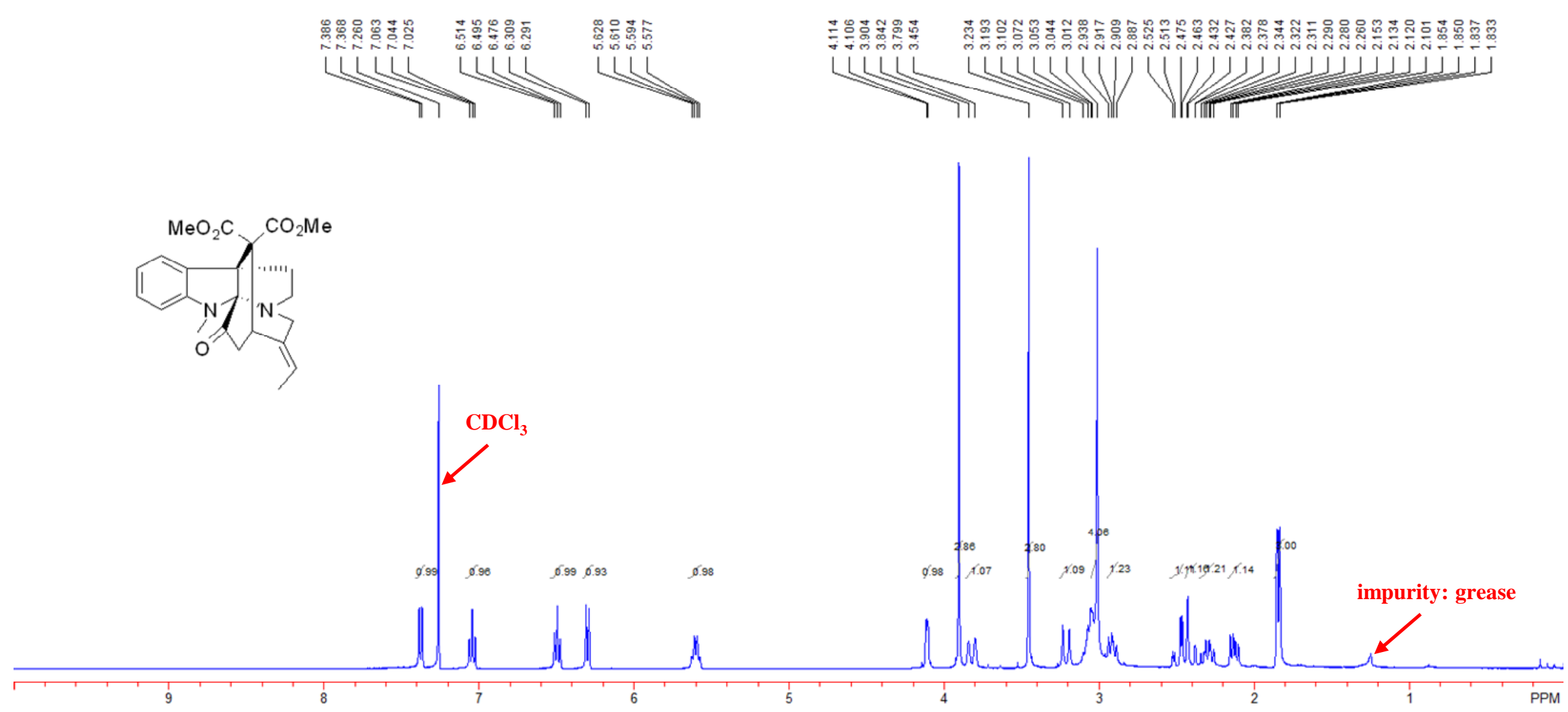
${ }^{13} \mathrm{C}$ NMR Spectrum of $7\left(101 \mathrm{MHz}, \mathrm{CDCl}_{3}\right)$
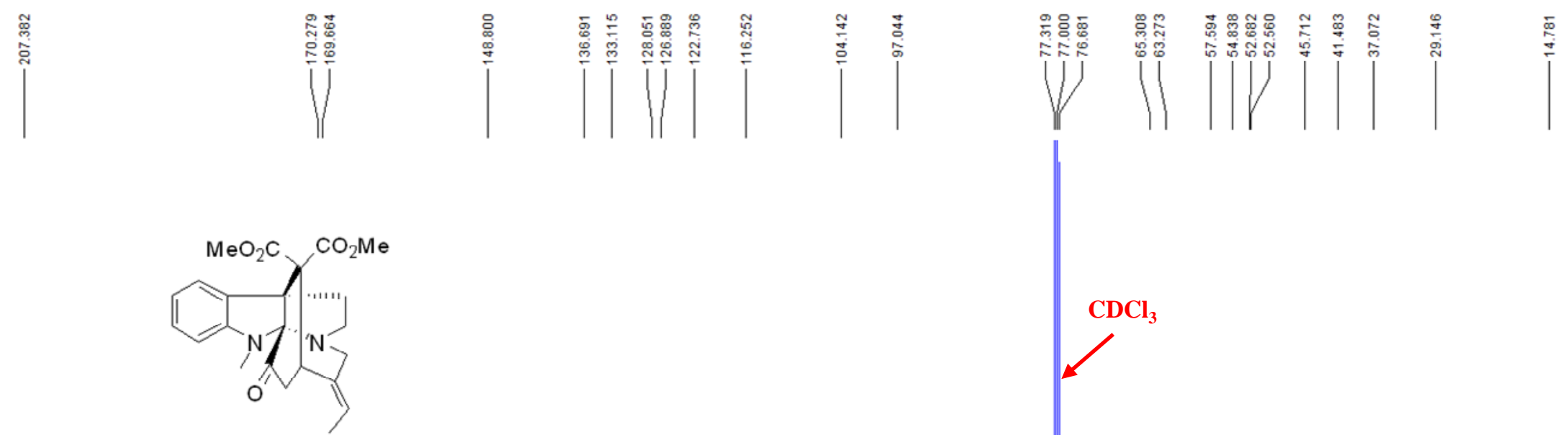

$\mathrm{CDCl}_{3}$

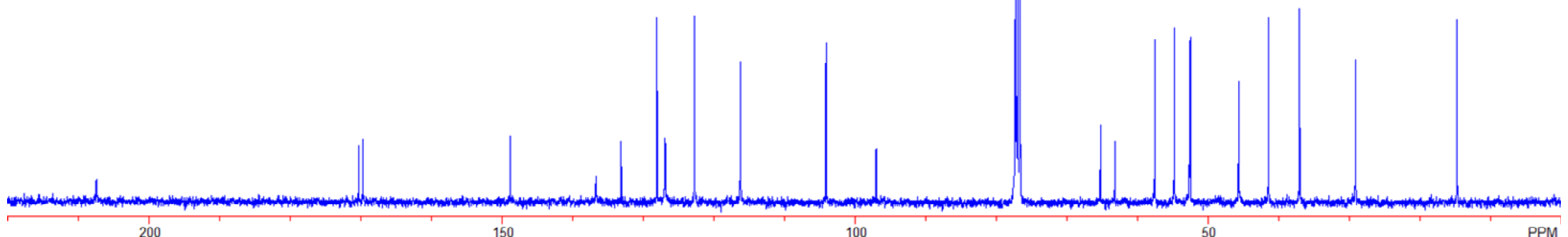


${ }^{1} \mathrm{H}$ NMR Spectrum of 19 (400 MHz, $\mathrm{CDCl}_{3}$ )

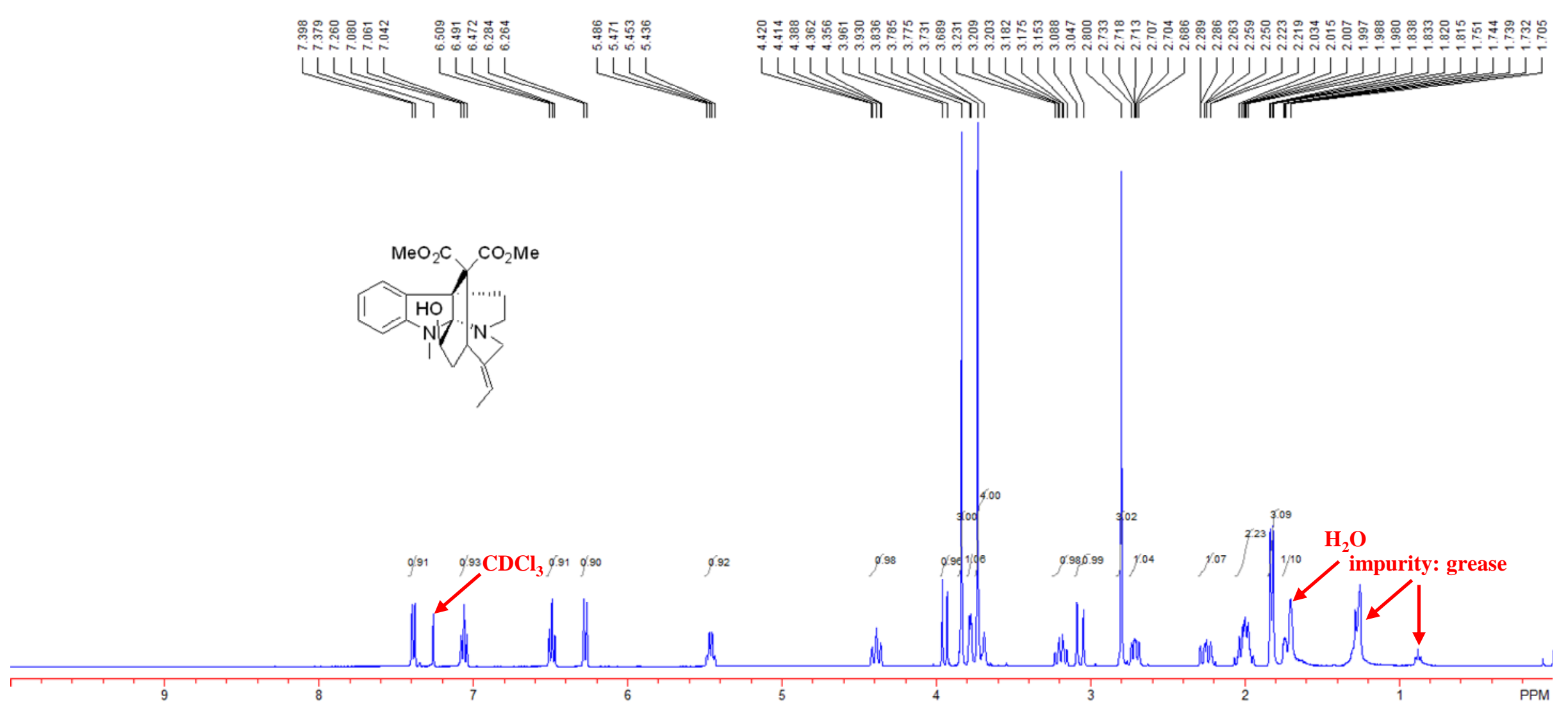


${ }^{13} \mathrm{C}$ NMR Spectrum of $19\left(101 \mathrm{MHz}, \mathrm{CDCl}_{3}\right)$
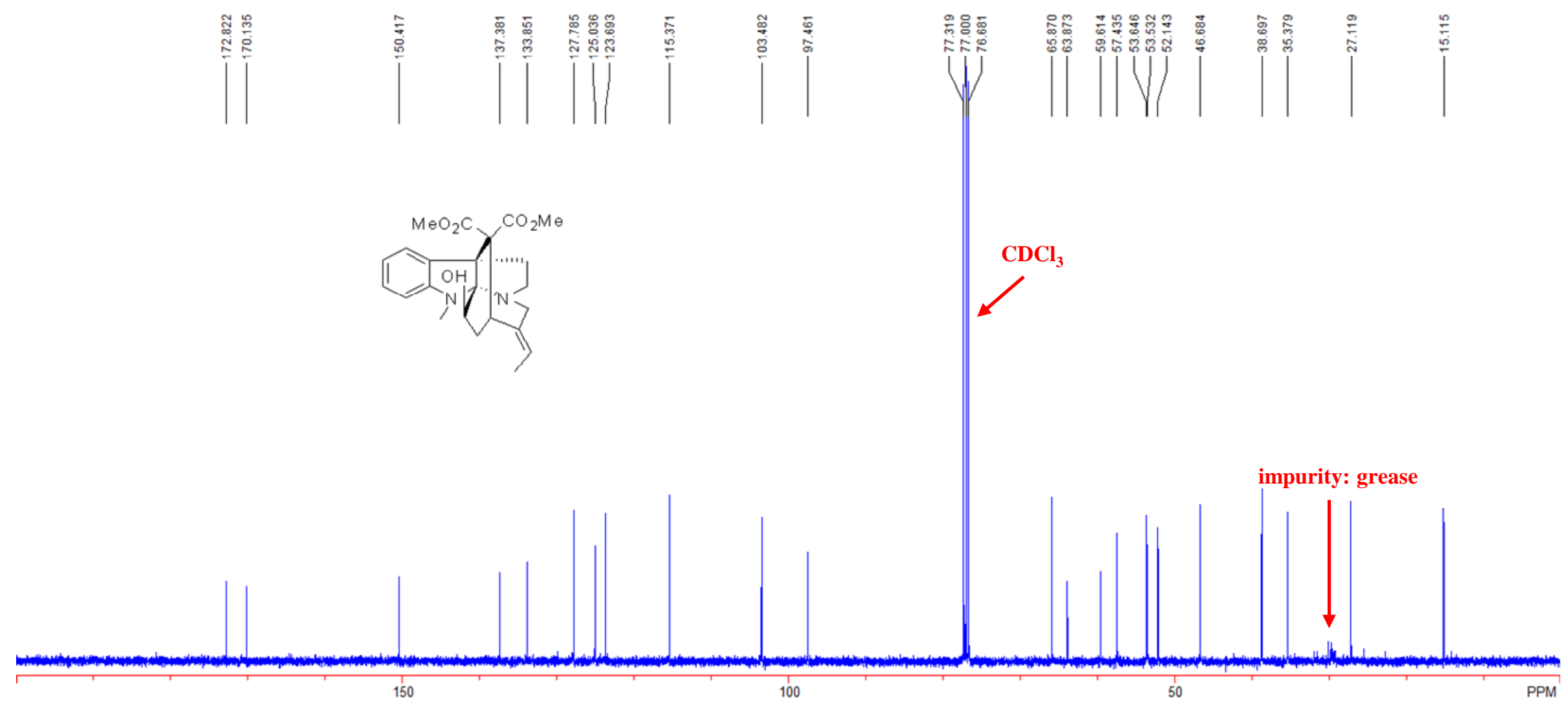
${ }^{1} \mathrm{H}$ NMR Spectrum of 5 (400 MHz, DMSO- $d_{6}$ )
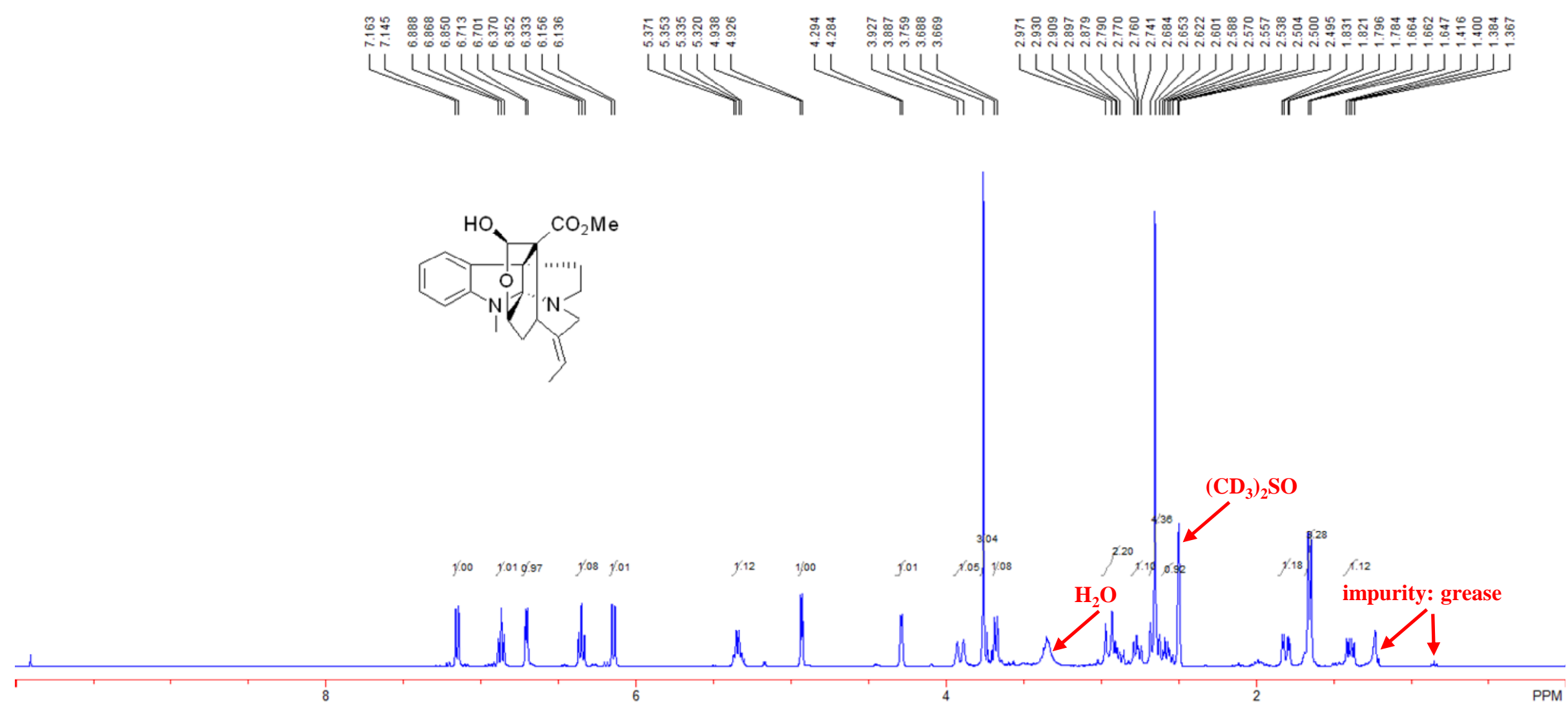
${ }^{13} \mathrm{C}$ NMR Spectrum of $5\left(101 \mathrm{MHz}, \mathrm{DMSO}-d_{6}\right)$

$$
\mid
$$
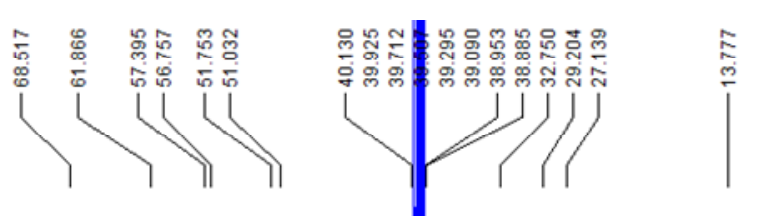
${ }^{1} \mathrm{H}$ NMR Spectrum of $6\left(400 \mathrm{MHz}, \mathrm{CDCl}_{3}\right)$

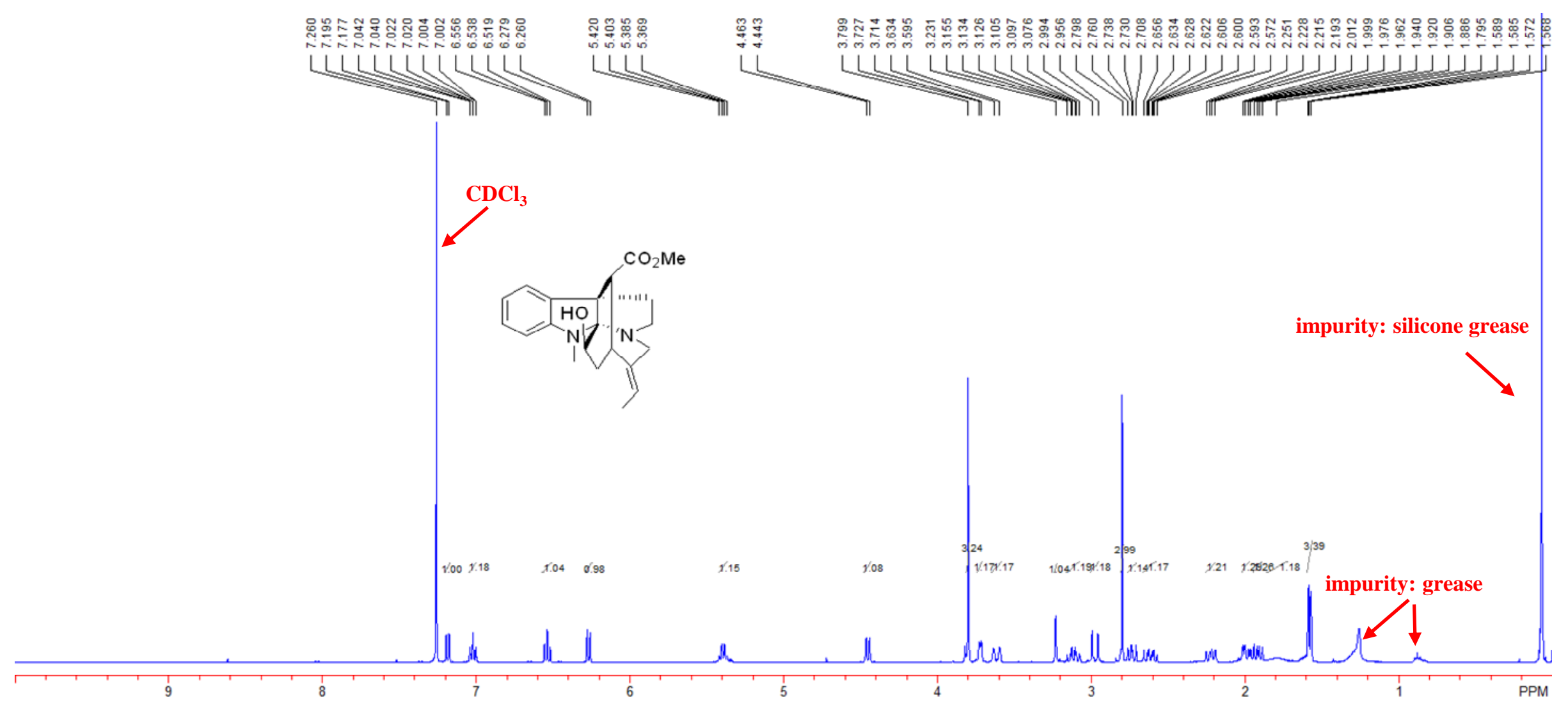


${ }^{13} \mathrm{C}$ NMR Spectrum of $6\left(101 \mathrm{MHz}, \mathrm{CDCl}_{3}\right)$

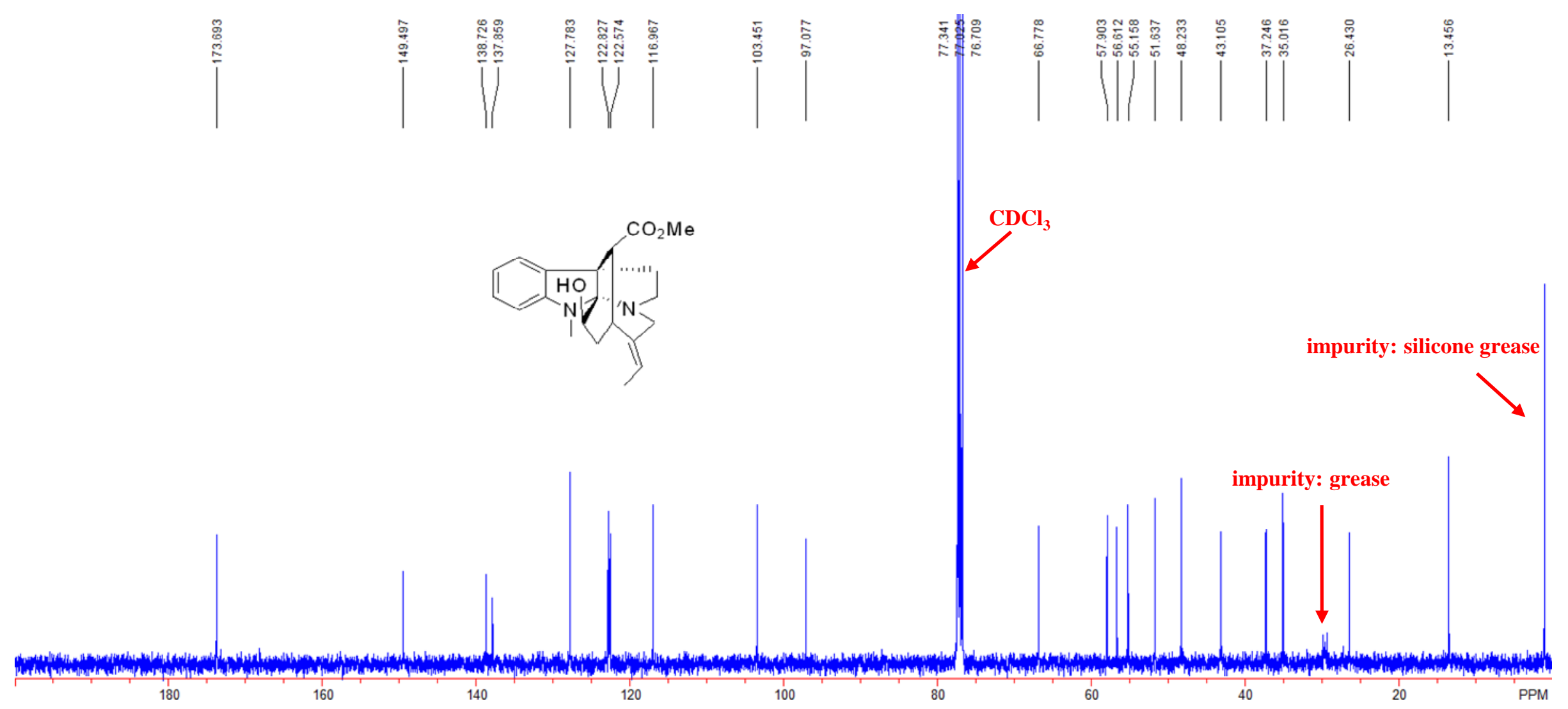


${ }^{1} \mathrm{H}$ NMR Spectrum of $20\left(400 \mathrm{MHz}, \mathrm{CDCl}_{3}\right)$

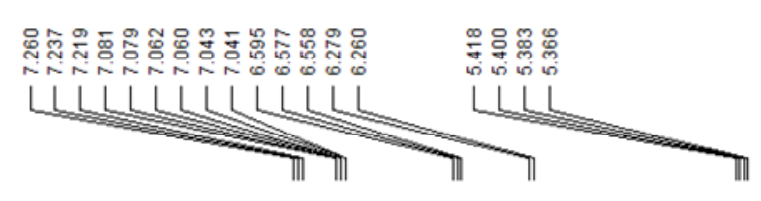

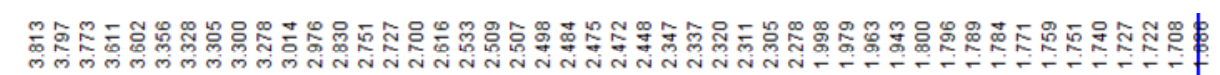

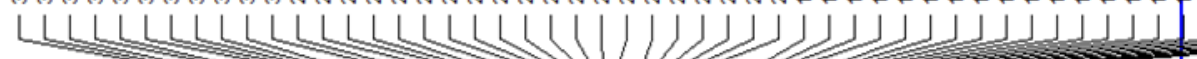
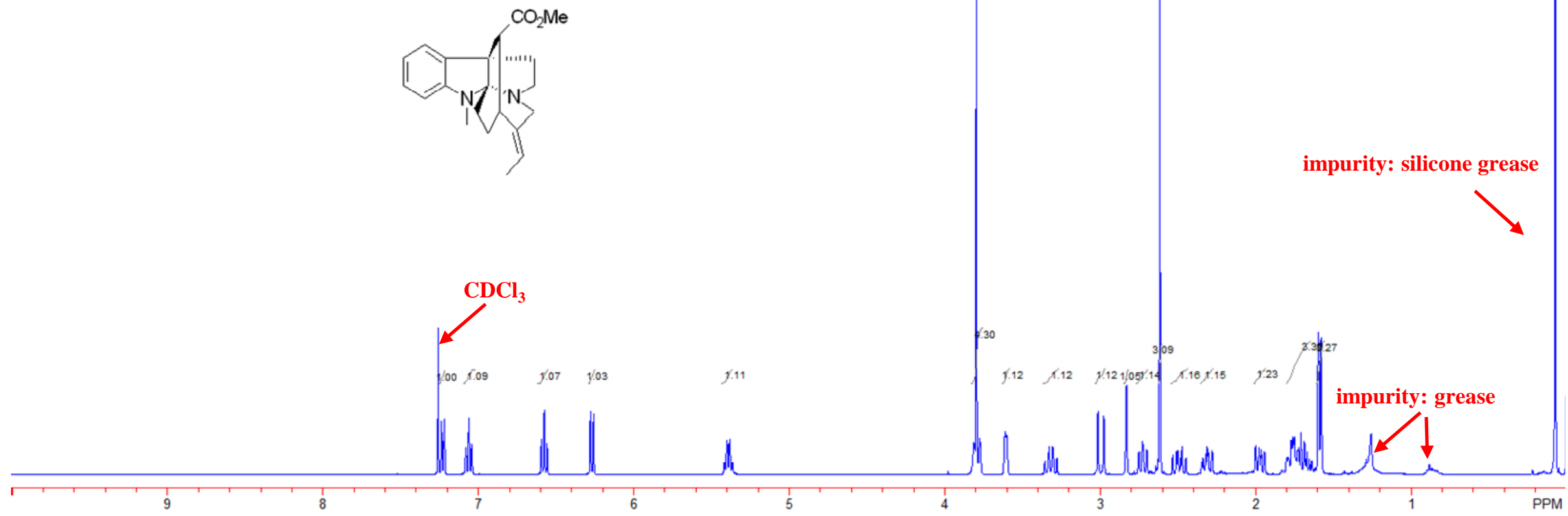
${ }^{13} \mathrm{C}$ NMR Spectrum of $20\left(101 \mathrm{MHz}, \mathrm{CDCl}_{3}\right)$
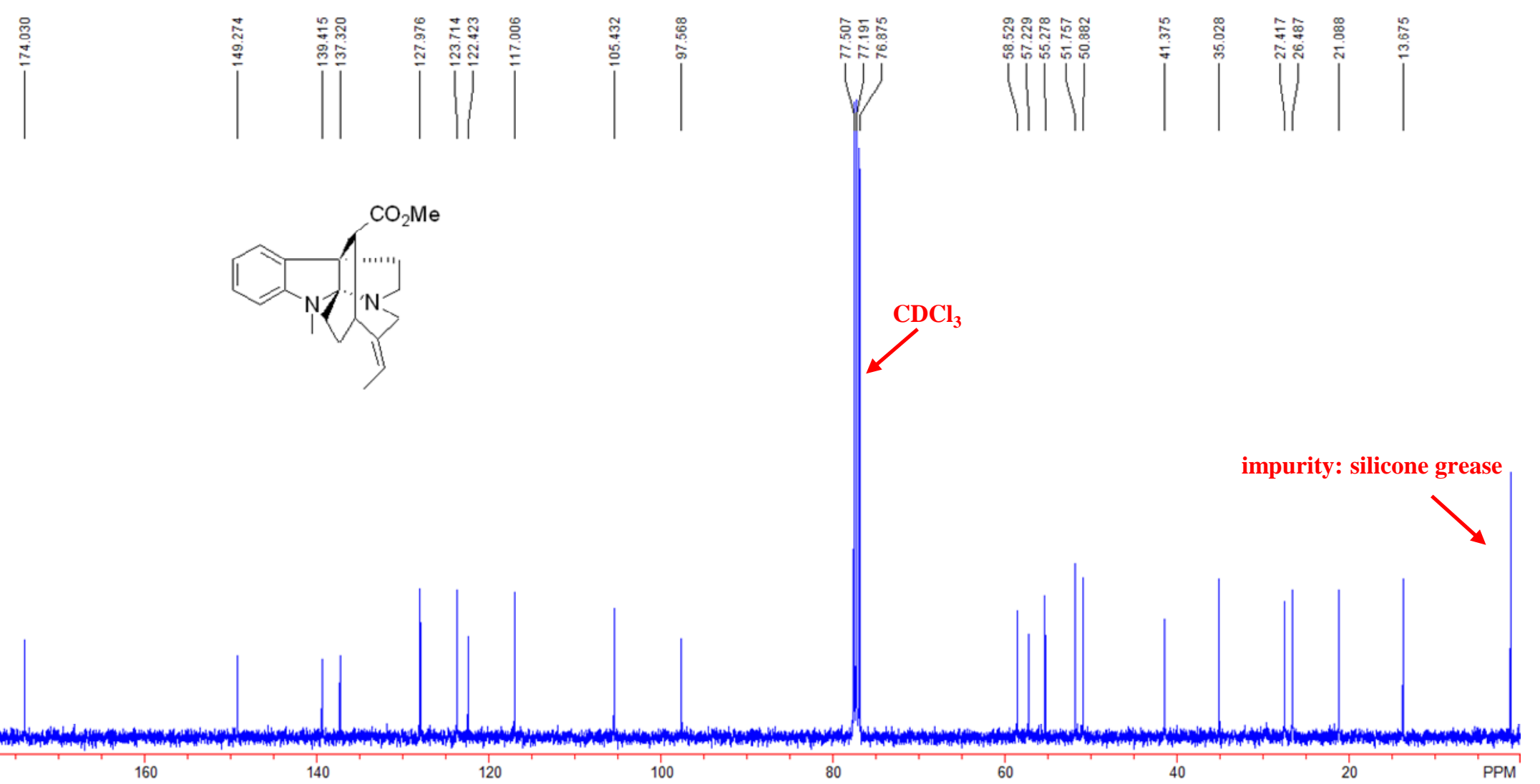
${ }^{1} \mathrm{H}$ NMR Spectrum of 21 (400 MHz, $\mathrm{CDCl}_{3}$ )

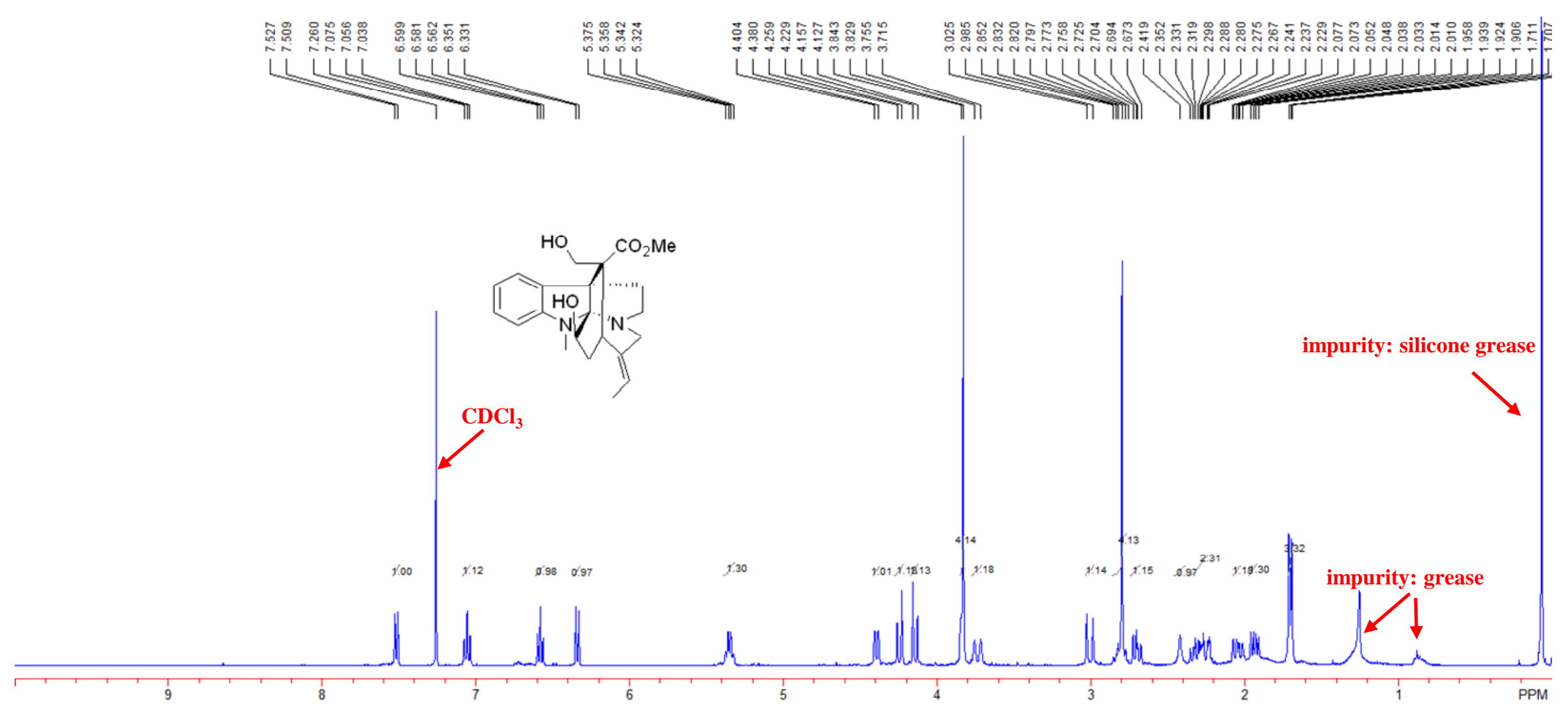


${ }^{13} \mathrm{C}$ NMR Spectrum of $21\left(101 \mathrm{MHz}, \mathrm{CDCl}_{3}\right)$

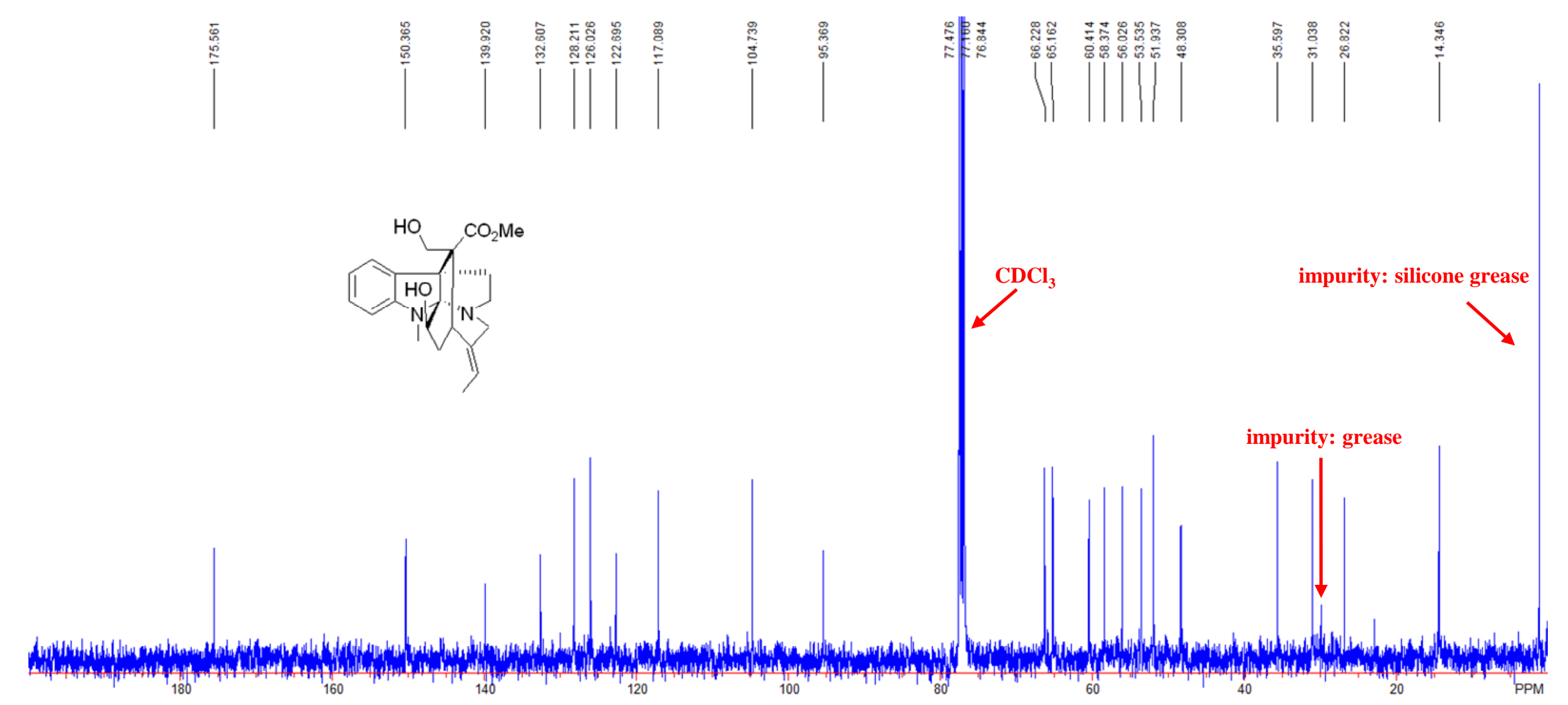


${ }^{1} \mathrm{H}$ NMR Spectrum of $22\left(400 \mathrm{MHz}, \mathrm{CDCl}_{3}\right.$ )
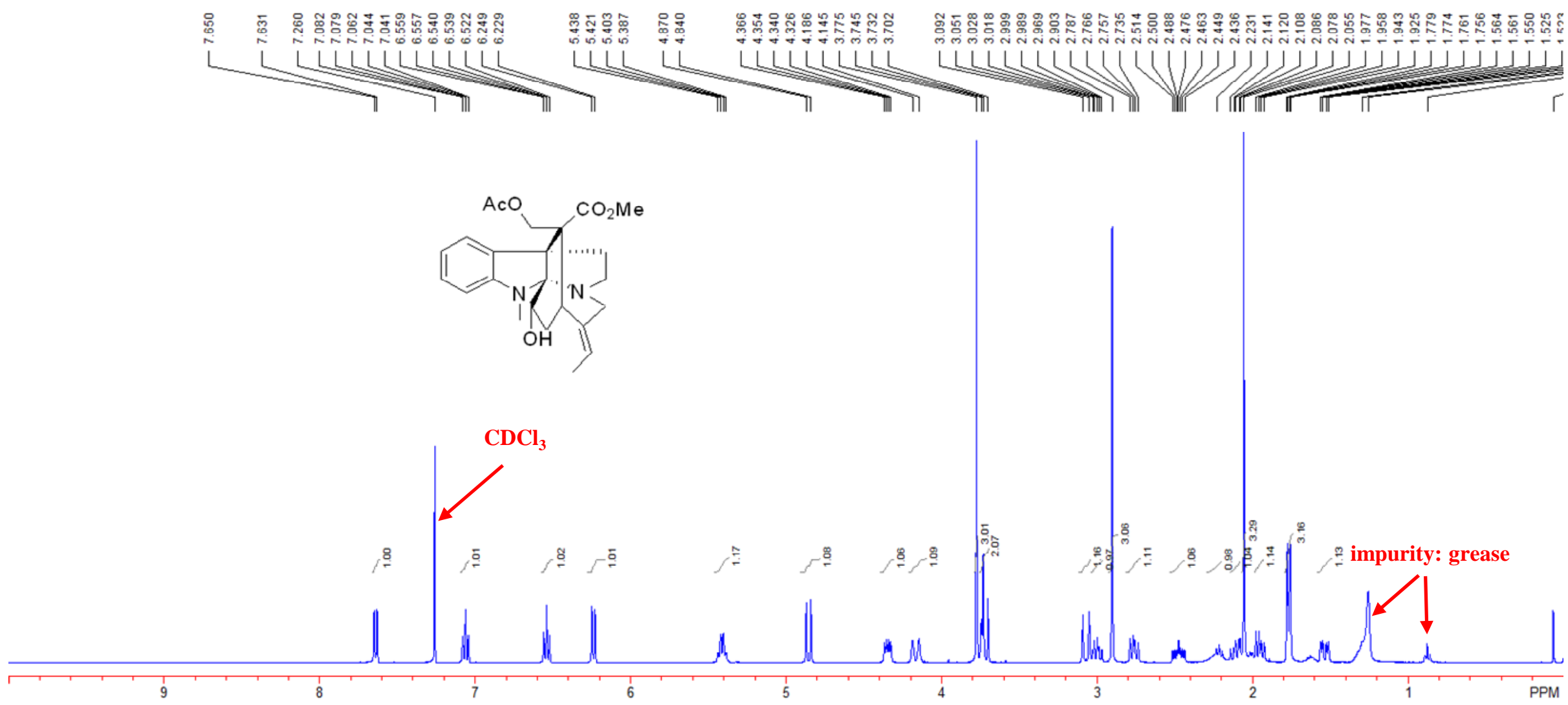
${ }^{13} \mathrm{C}$ NMR Spectrum of $22\left(101 \mathrm{MHz}, \mathrm{CDCl}_{3}\right)$
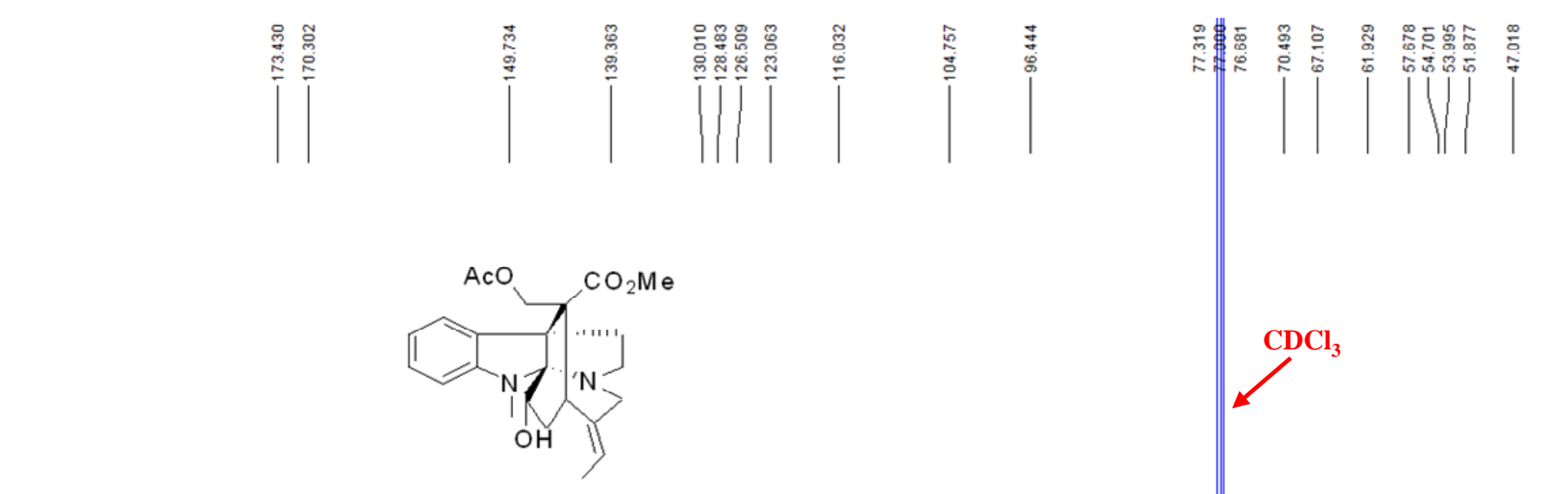

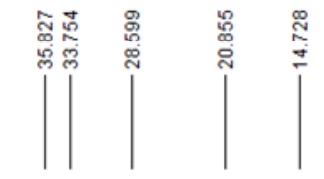

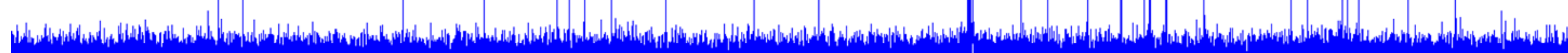


${ }^{1} \mathrm{H}$ NMR Spectrum of $23\left(400 \mathrm{MHz}, \mathrm{CDCl}_{3}\right.$ )

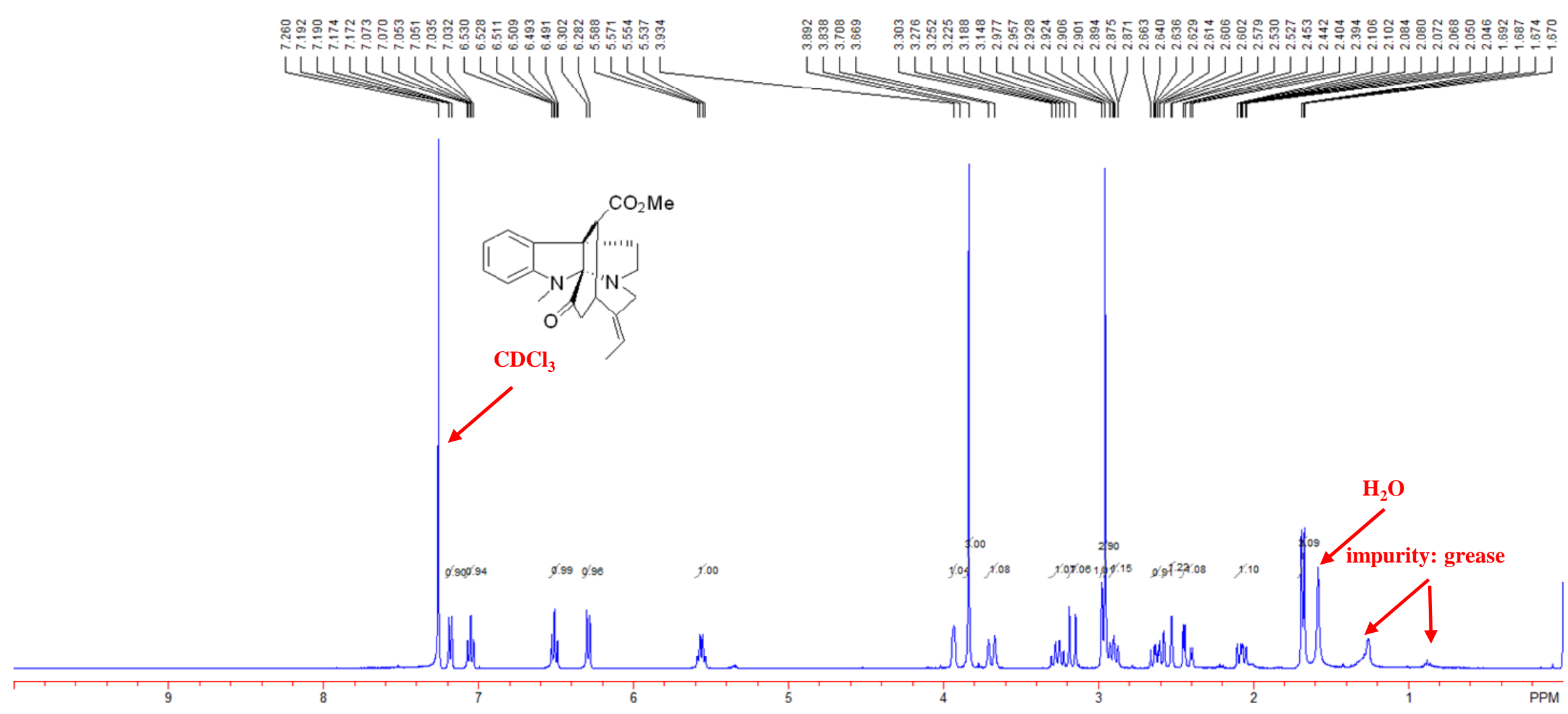


${ }^{13} \mathrm{C}$ NMR Spectrum of $23\left(101 \mathrm{MHz}, \mathrm{CDCl}_{3}\right)$

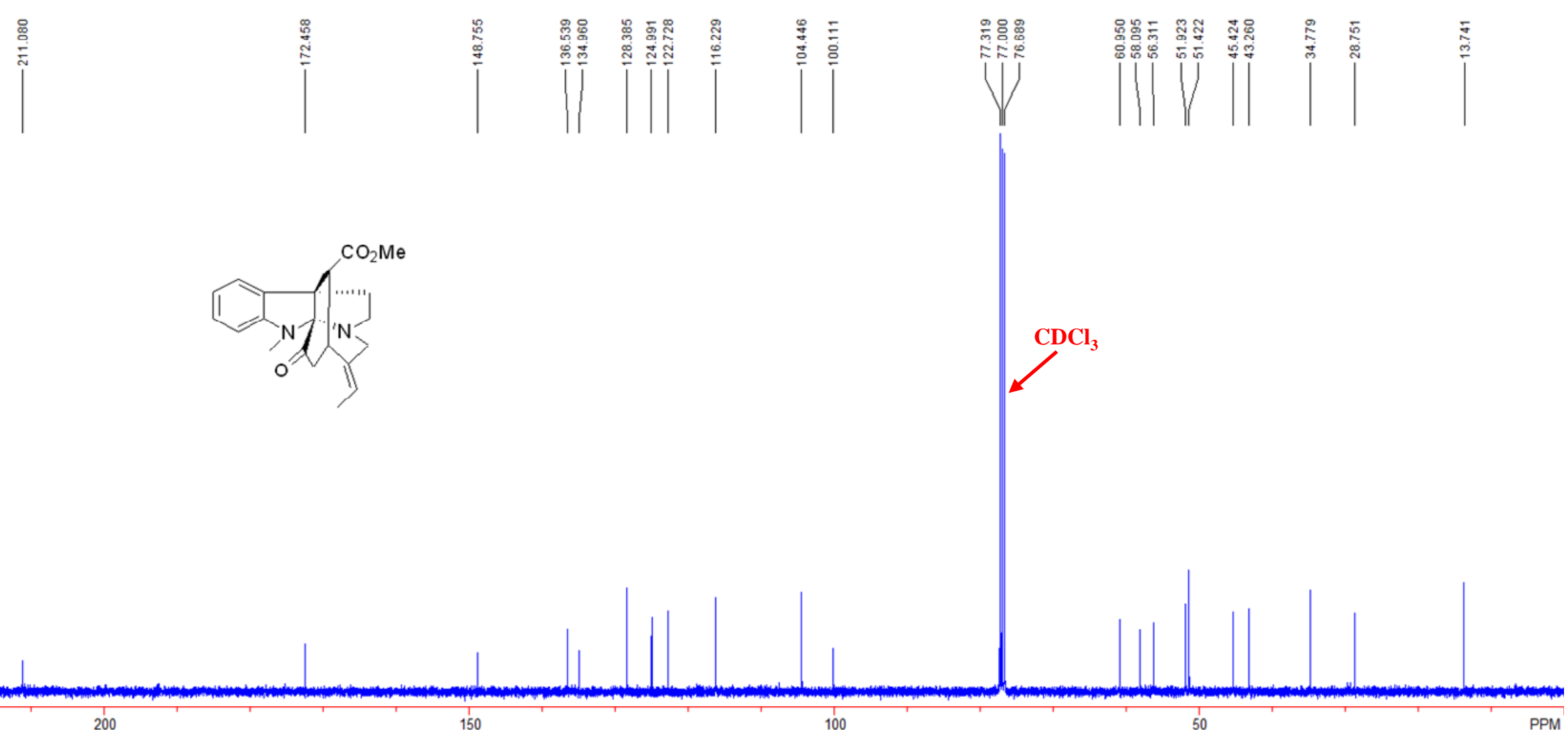


${ }^{1} \mathrm{H}$ NMR Spectrum of $24\left(400 \mathrm{MHz}, \mathrm{CDCl}_{3}\right)$

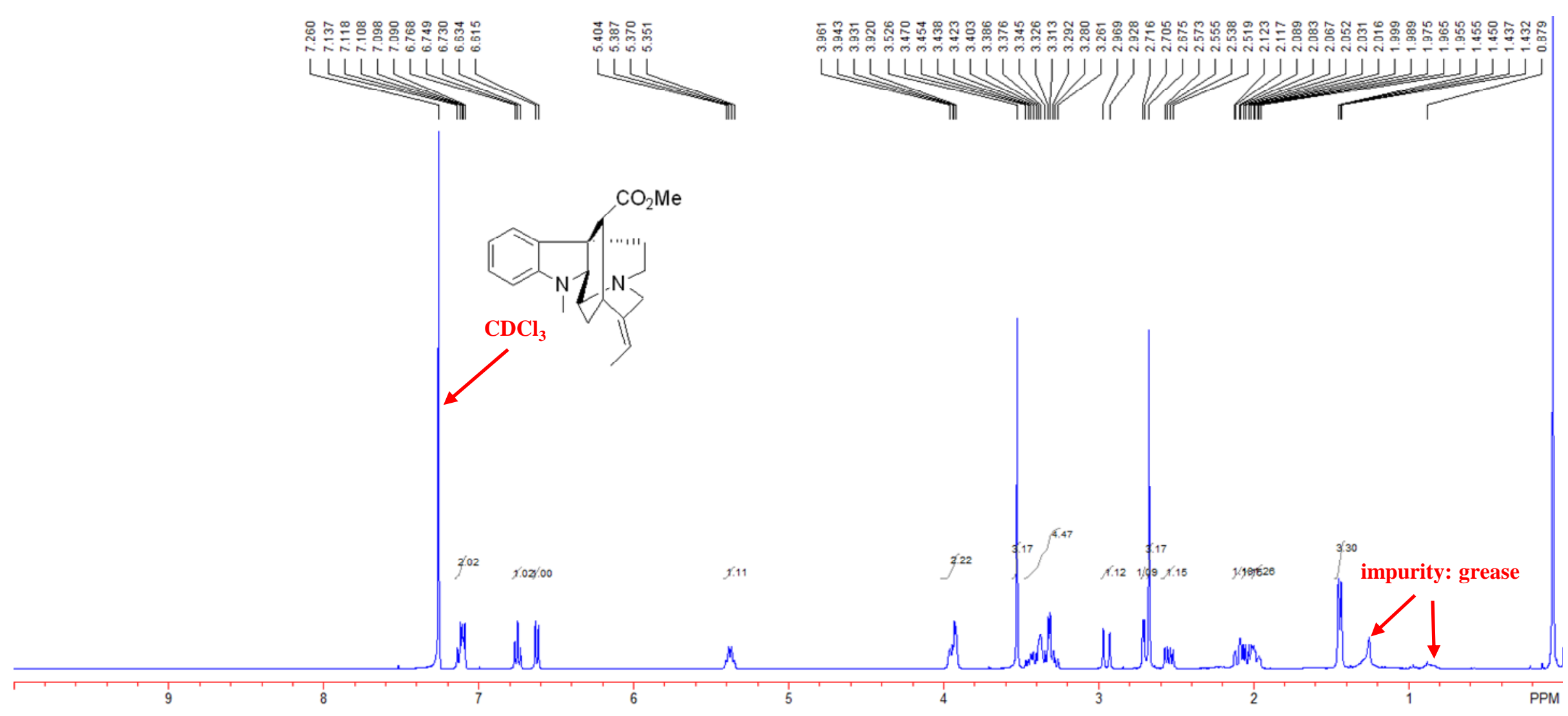


${ }^{13} \mathrm{C}$ NMR Spectrum of $24\left(101 \mathrm{MHz}, \mathrm{CDCl}_{3}\right)$

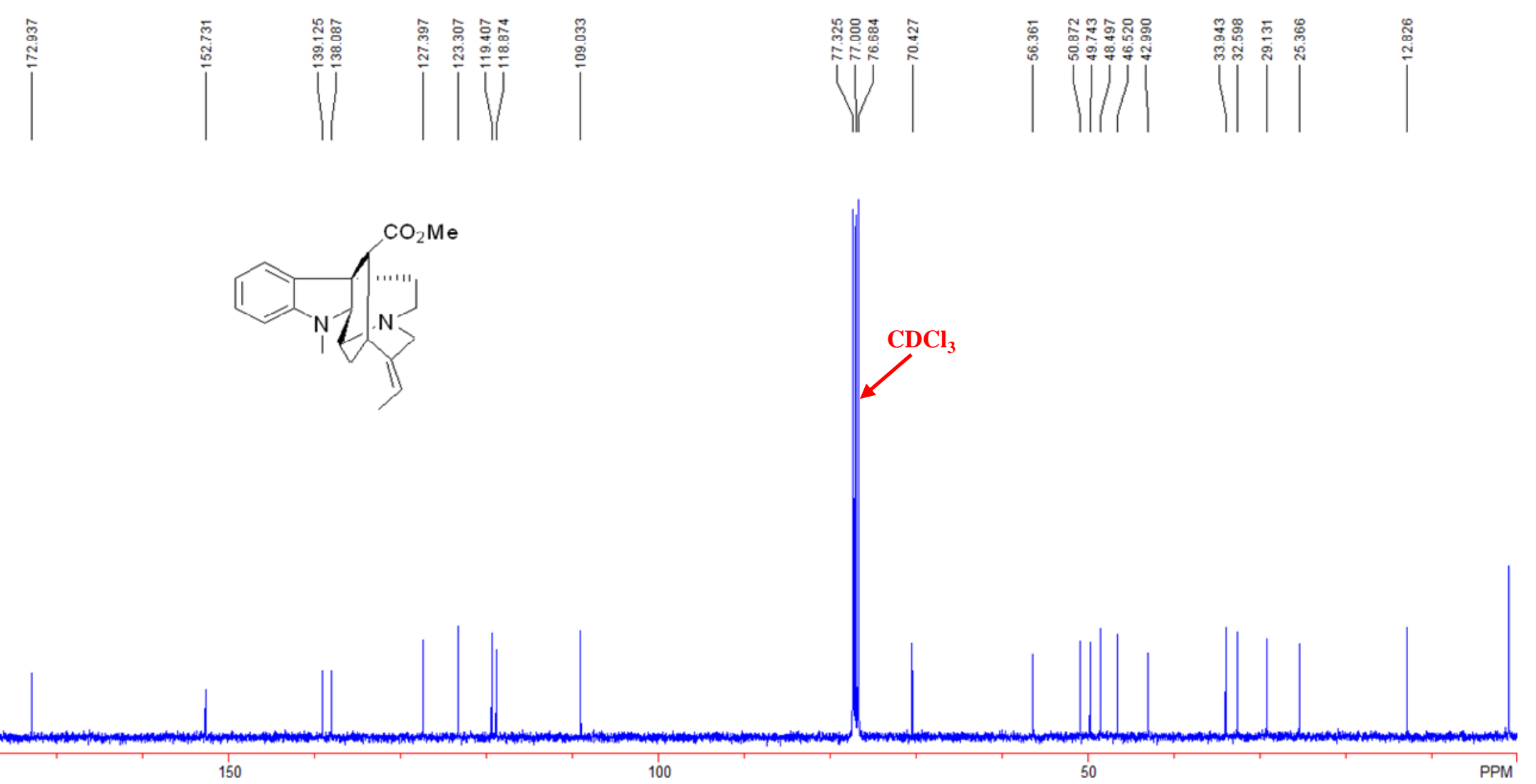


${ }^{1} \mathrm{H}$ NMR Spectrum of SI-1 (400 MHz, $\left.\mathrm{CDCl}_{3}\right)$
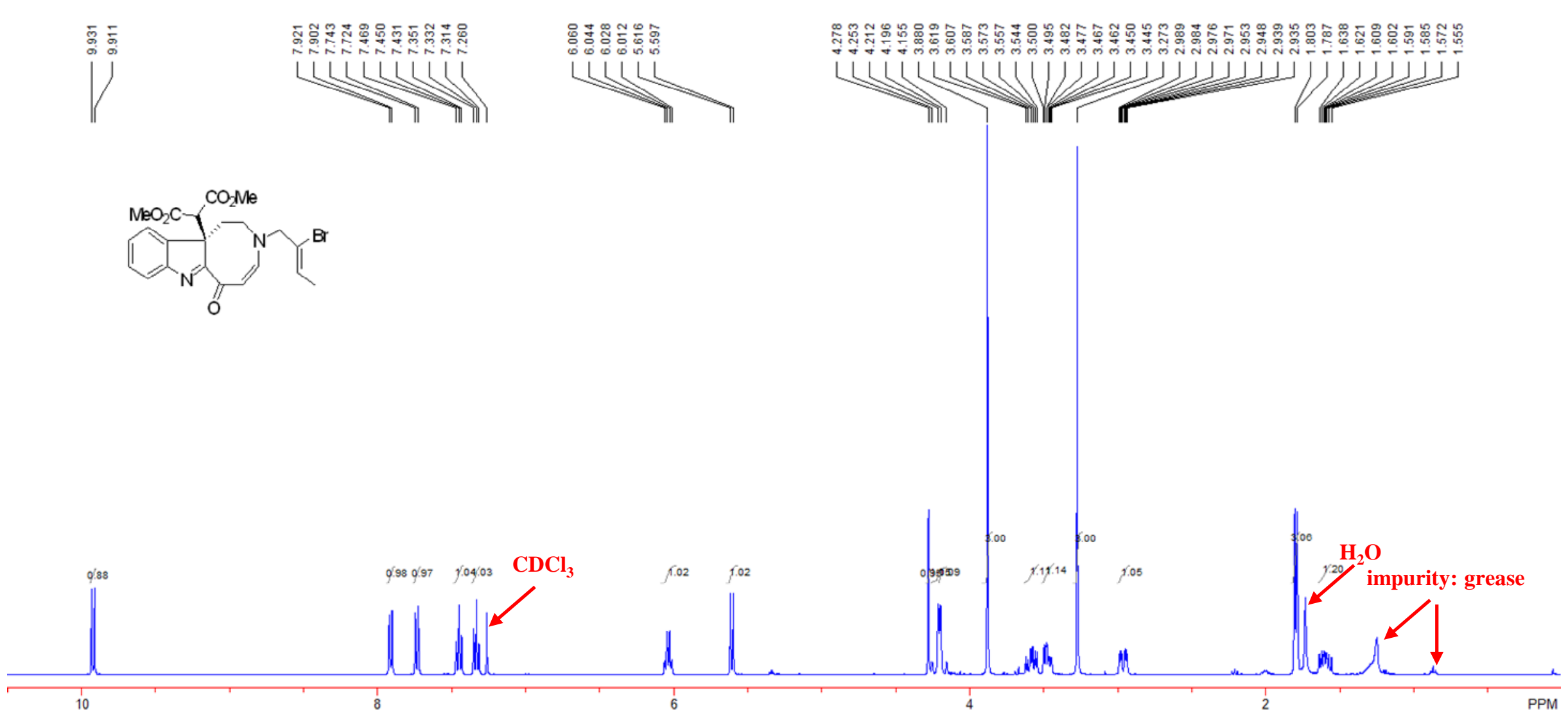
${ }^{13} \mathrm{C}$ NMR Spectrum of SI-1 (101 MHz, $\left.\mathrm{CDCl}_{3}\right)$

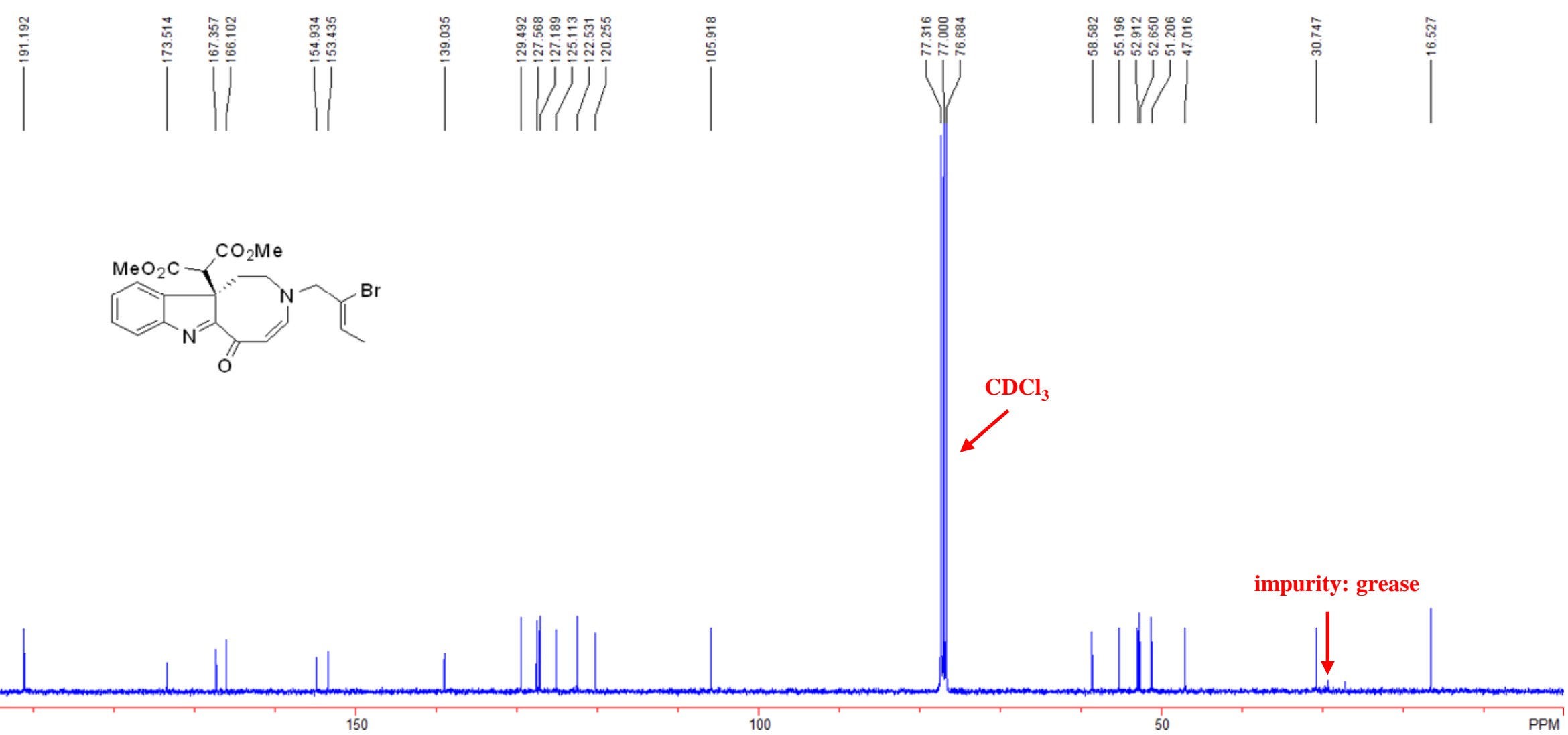


${ }^{1} \mathrm{H}$ NMR Spectrum of SI-2 (400 MHz, $\left.\mathrm{CDCl}_{3}\right)$
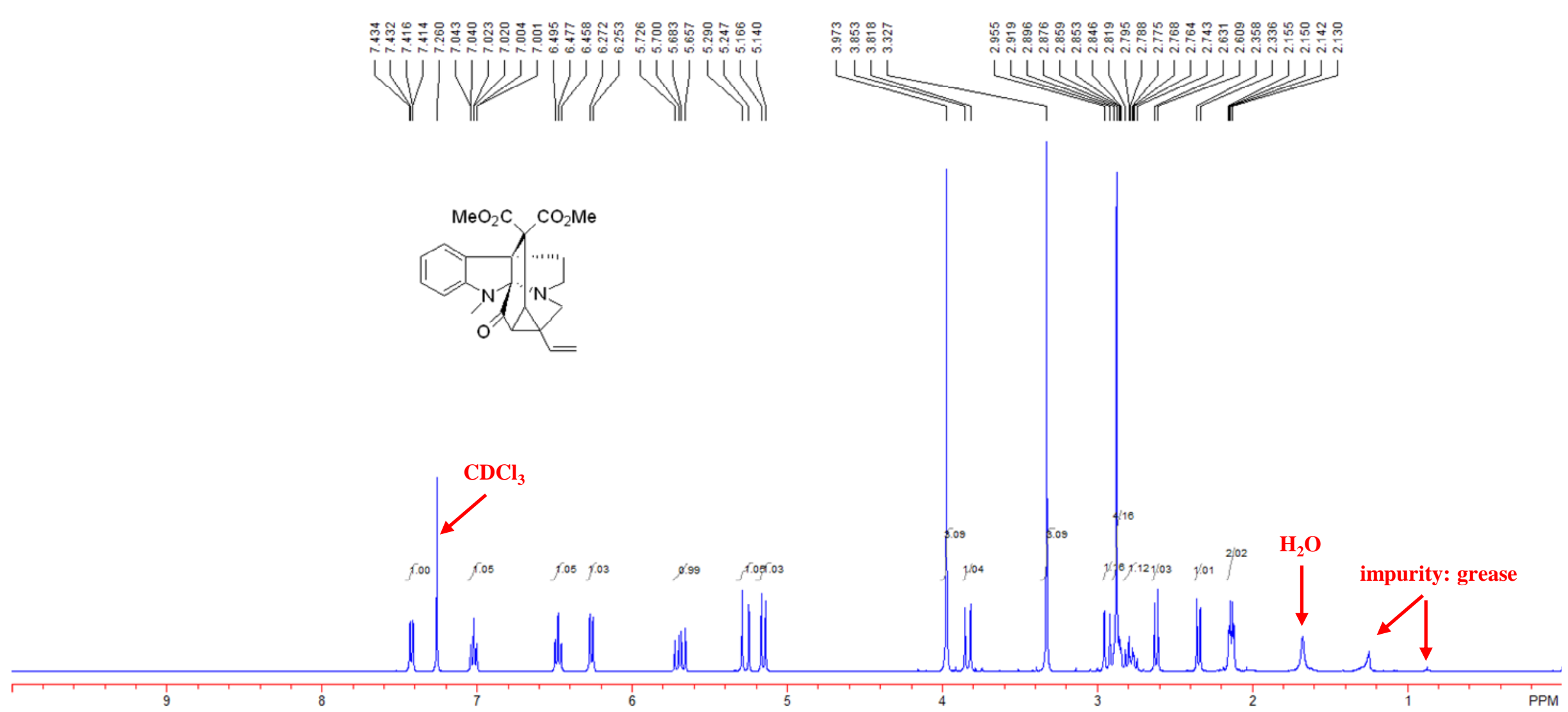
${ }^{13} \mathrm{C}$ NMR Spectrum of SI-2 (101 MHz, $\mathrm{CDCl}_{3}$ )

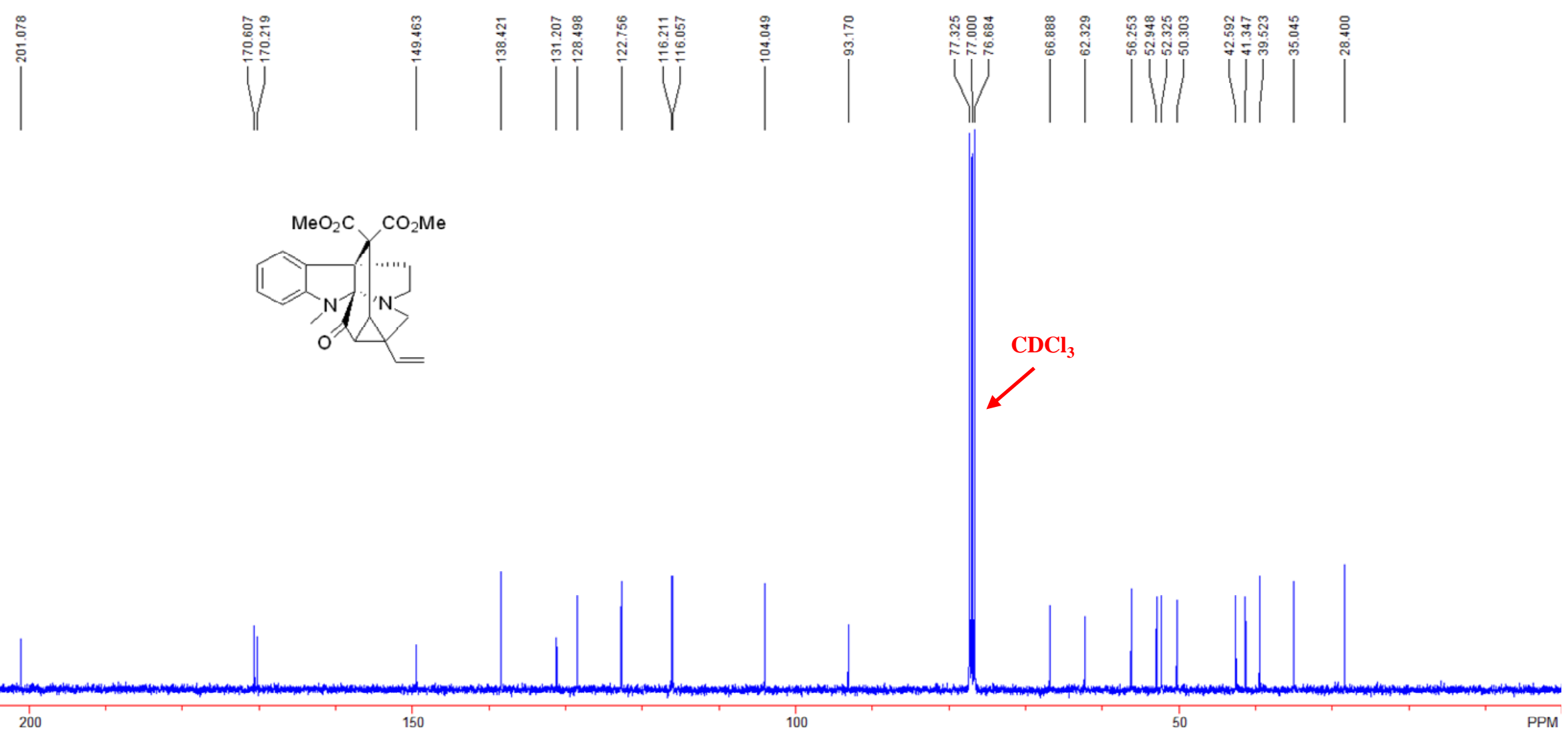


6. Comparison of spectra and data of natural and synthetic corymine and analogues

(a) Corymine, comparison of spectra

Our's ( ${ }^{1} \mathrm{H}$ NMR $\left(400 \mathrm{MHz}\right.$, DMSO- $\left.d_{6}\right),(+)$-corymine $)$
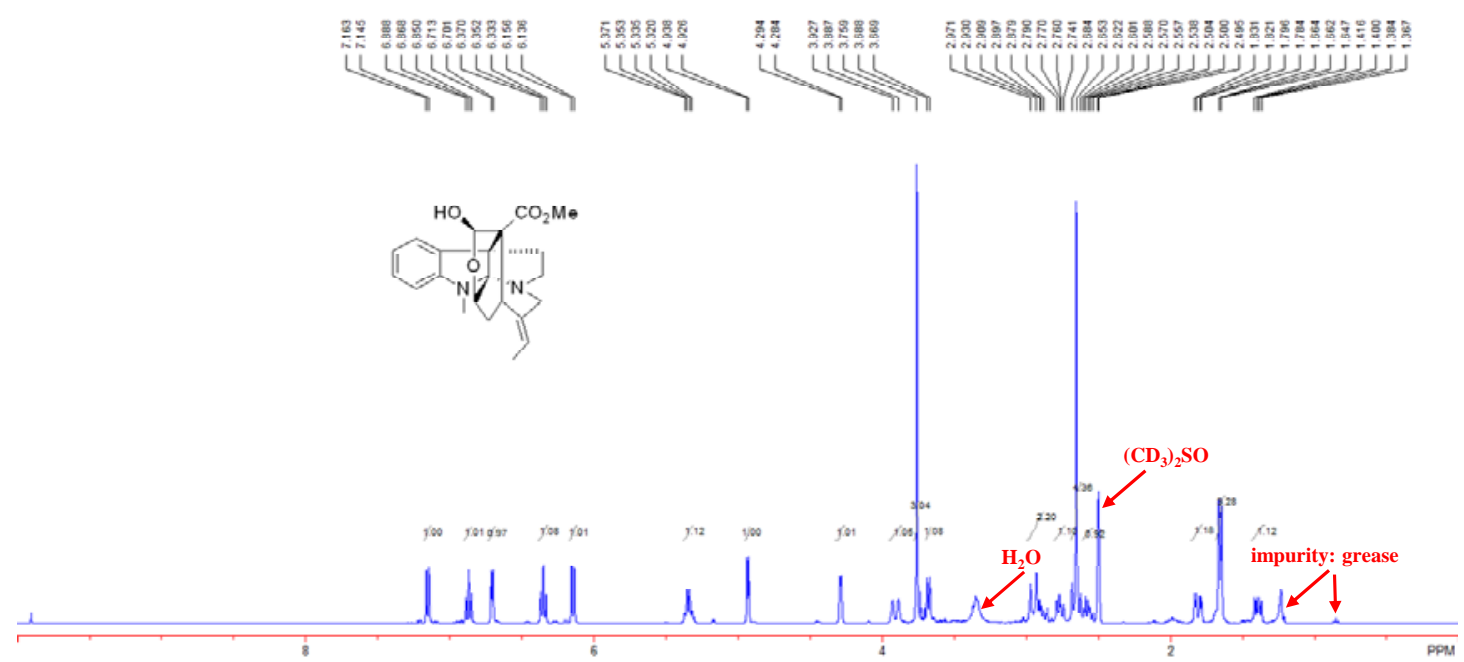

Our's ( ${ }^{1} \mathrm{H}$ NMR (400 MHz, DMSO- $\left.d_{6}\right),( \pm)$-corymine) (Angew. Chem., Int. Ed. 2017, $56,7484)$
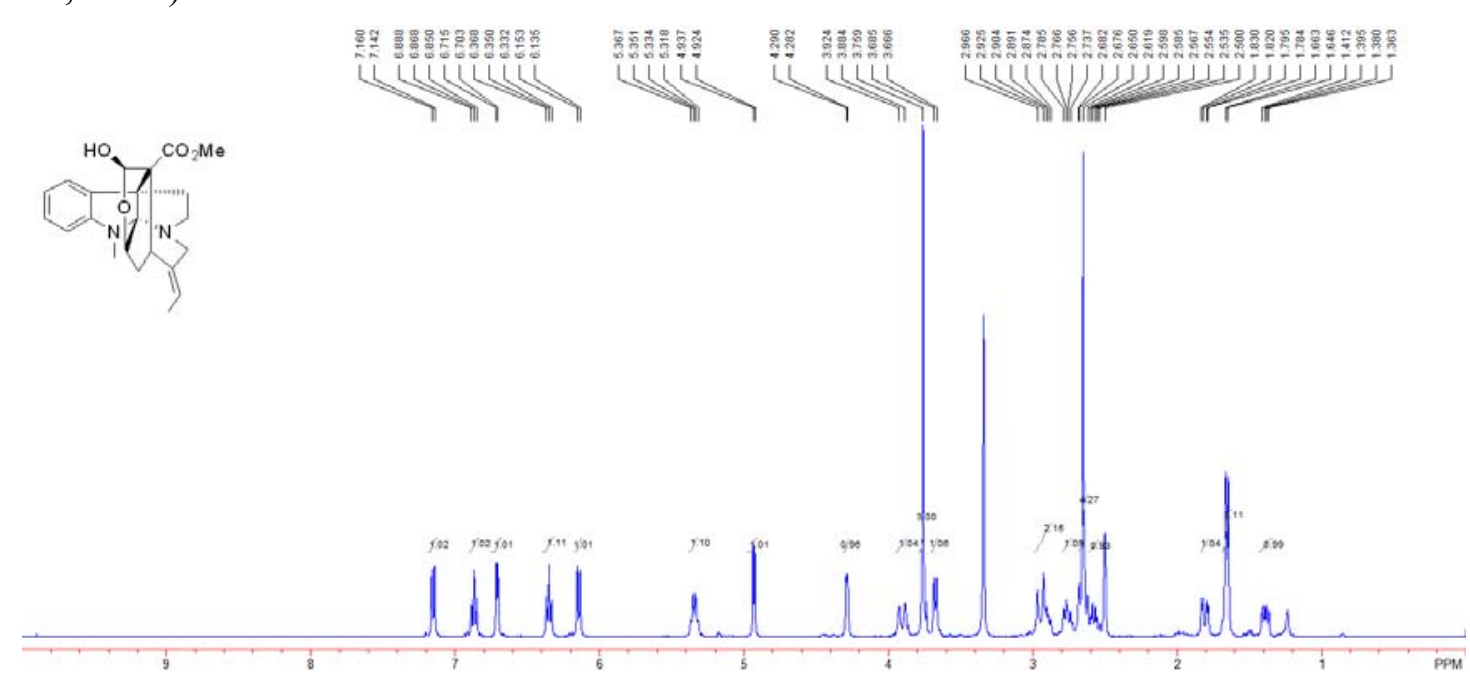

The ${ }^{1} \mathrm{H}$ NMR spectrum of natural corymine is not available. 
Our's $\left({ }^{13} \mathrm{C}\right.$ NMR $\left(101 \mathrm{MHz}, \mathrm{DMSO}-d_{6}\right),(+)$-corymine $)$

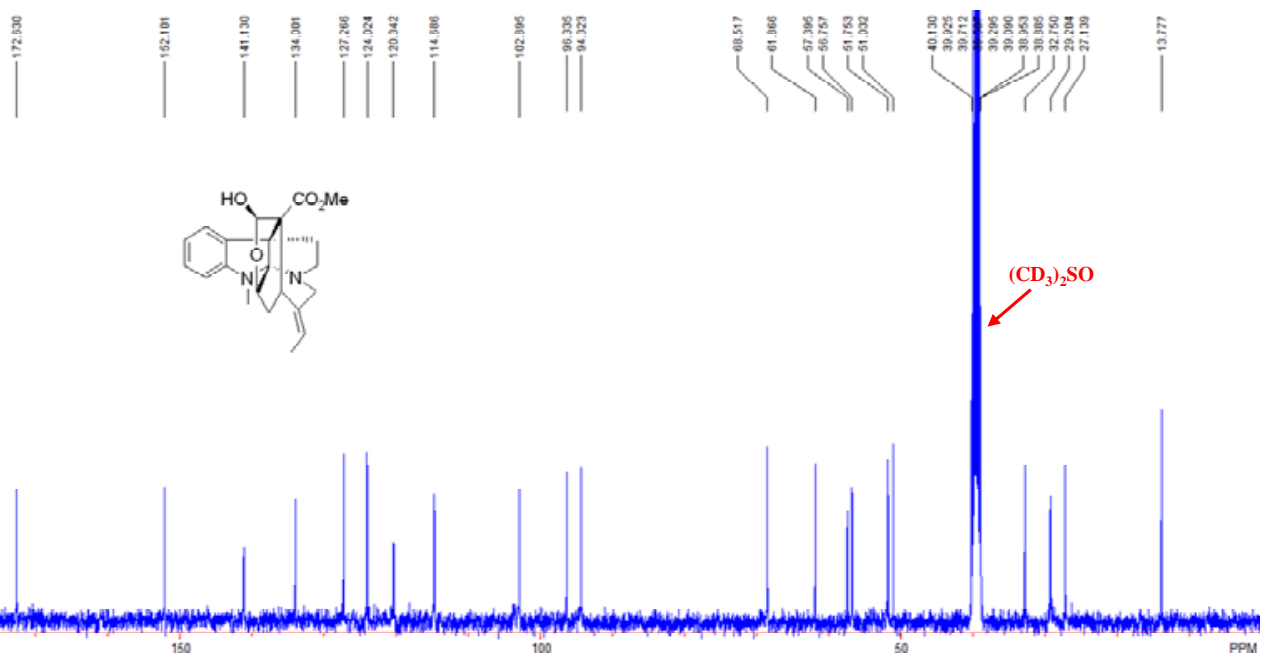

Our's $\left.\left({ }^{13} \mathrm{C} \text { NMR (101 MHz, DMSO-d }\right)_{6}\right),( \pm)$-corymine) (Angew. Chem., Int. Ed. 2017, $56,7484)$

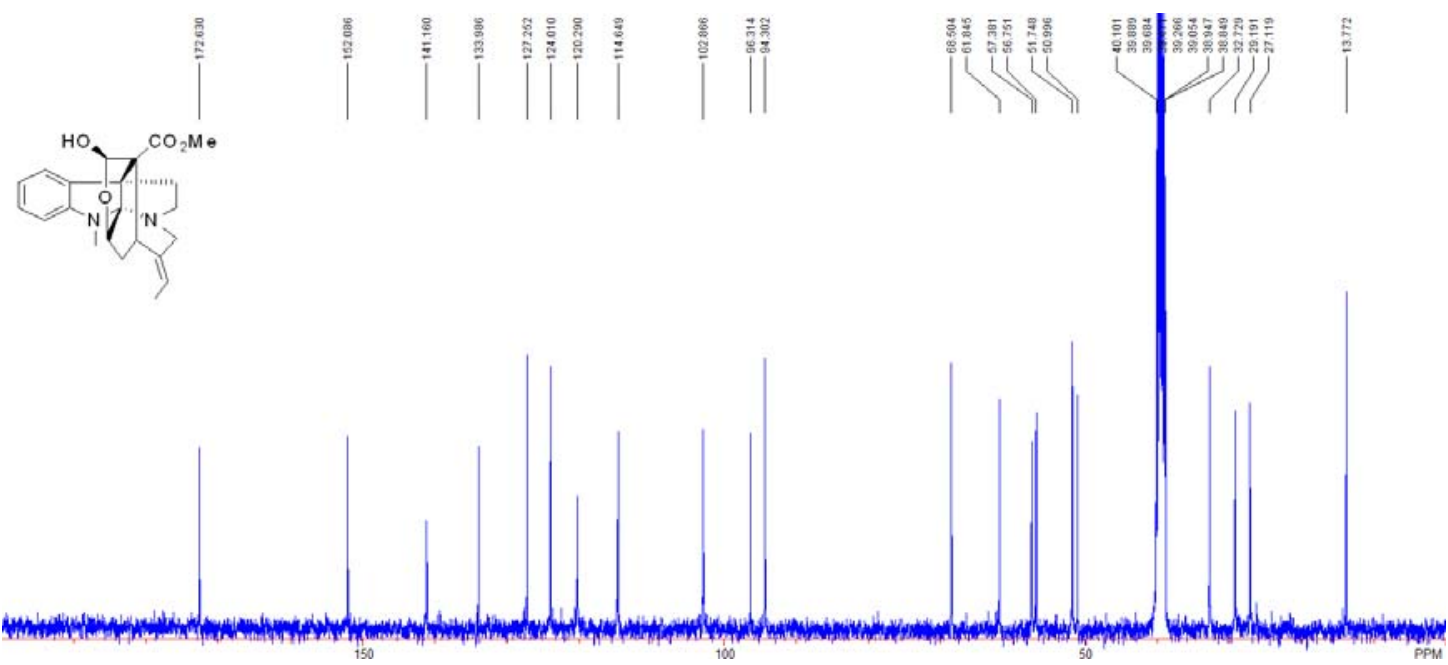

The ${ }^{13} \mathrm{C}$ NMR spectrum of natural corymine is not available. The reported data are copied as follows:

\begin{tabular}{cccr}
\hline \hline Carbon & $\mathbf{1}^{\text {a) }}$ & Carbon & \multicolumn{1}{c}{$\mathbf{1}^{\text {a) }}$} \\
\hline 2 & 96.31 & 14 & 29.21 \\
3 & 68.52 & 15 & 32.75 \\
5 & 56.75 & 16 & 51.73 \\
6 & 39.00 & 17 & 94.32 \\
7 & 61.87 & 18 & 13.76 \\
8 & 134.00 & 19 & 120.25 \\
9 & 124.01 & 20 & 141.18 \\
10 & 114.63 & 21 & 57.41 \\
11 & 127.24 & $\mathrm{CO}$ & 172.63 \\
12 & 102.85 & OMe & 51.73 \\
13 & 152.09 & $\mathrm{NMe}$ & 27.10 \\
\hline a) In DMSO- $d_{\text {6. }}$ & & &
\end{tabular}




\section{(b) Deformylcorymine, comparison of spectra}

Our's ( ${ }^{1} \mathrm{H}$ NMR (400 MHz, $\left.\mathrm{CDCl}_{3}\right),(-)$-deformylcorymine)

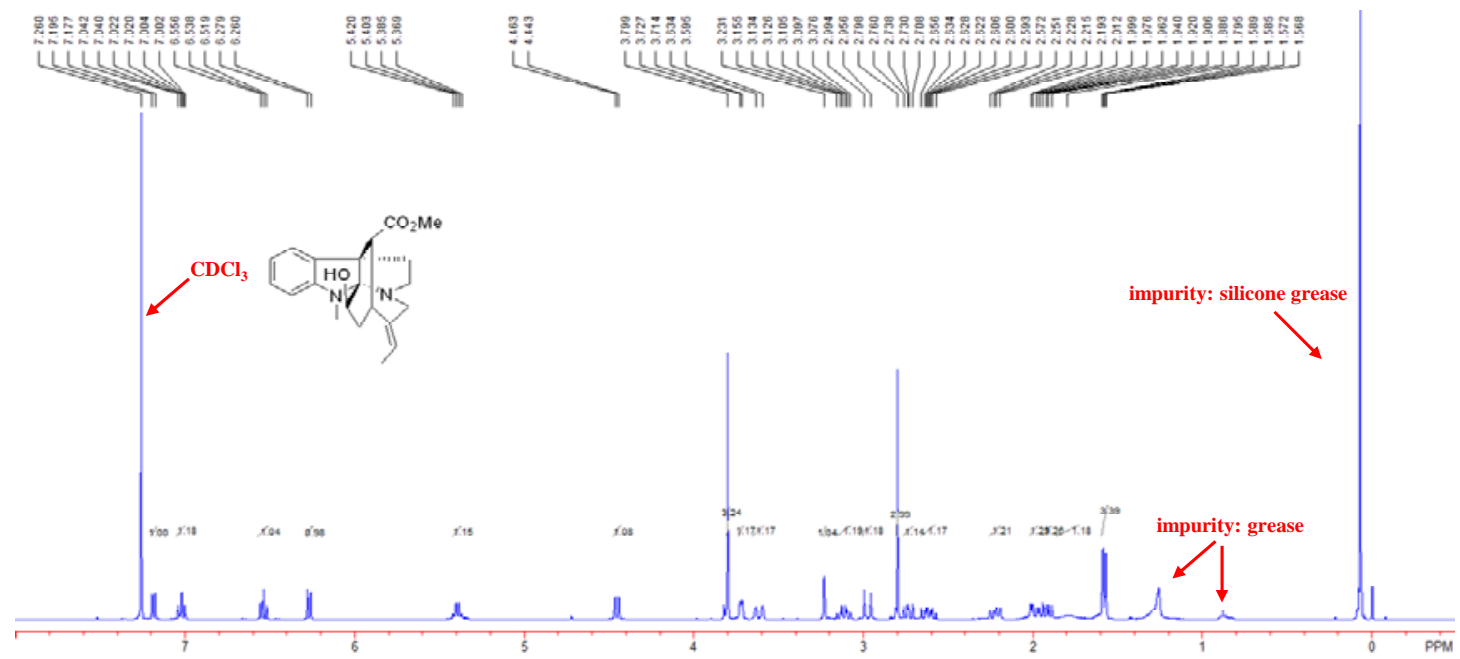

Zu's ( ${ }^{1} \mathrm{H}$ NMR (400 MHz, $\left.\mathrm{CDCl}_{3}\right),( \pm)$-deformylcorymine) (Org. Lett. 2017, 19, 5430)

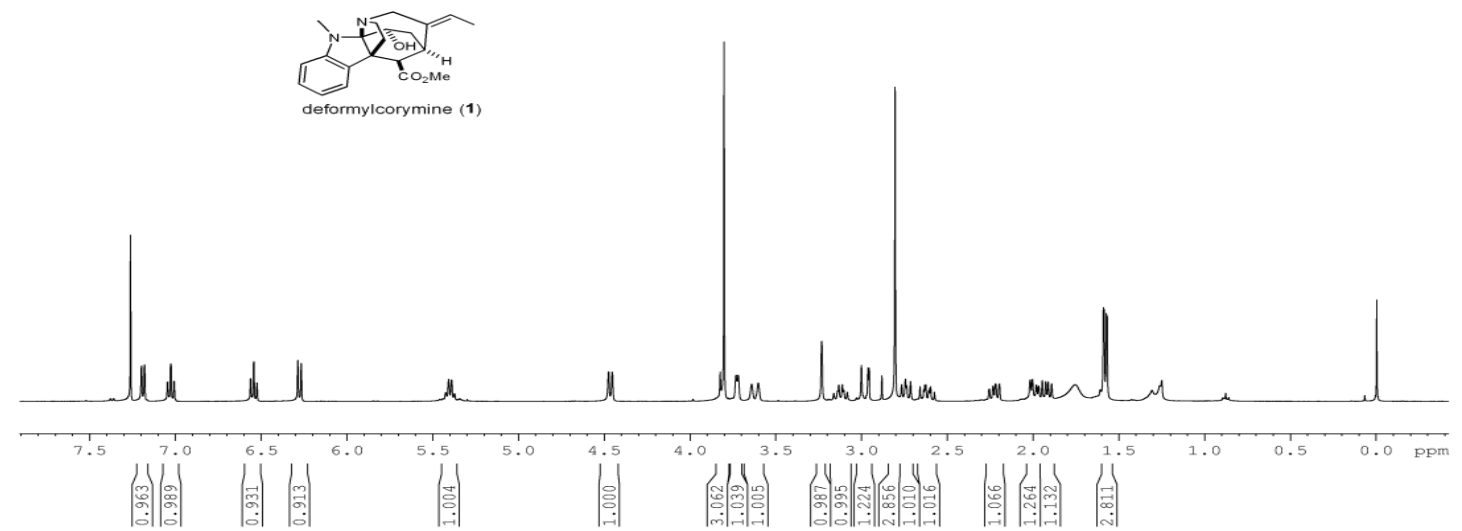

The ${ }^{1} \mathrm{H}$ NMR spectrum of natural deformylcorymine is not available. 
Our's $\left({ }^{13} \mathrm{C}\right.$ NMR $\left(101 \mathrm{MHz}, \mathrm{CDCl}_{3}\right),(-)$-deformylcorymine)

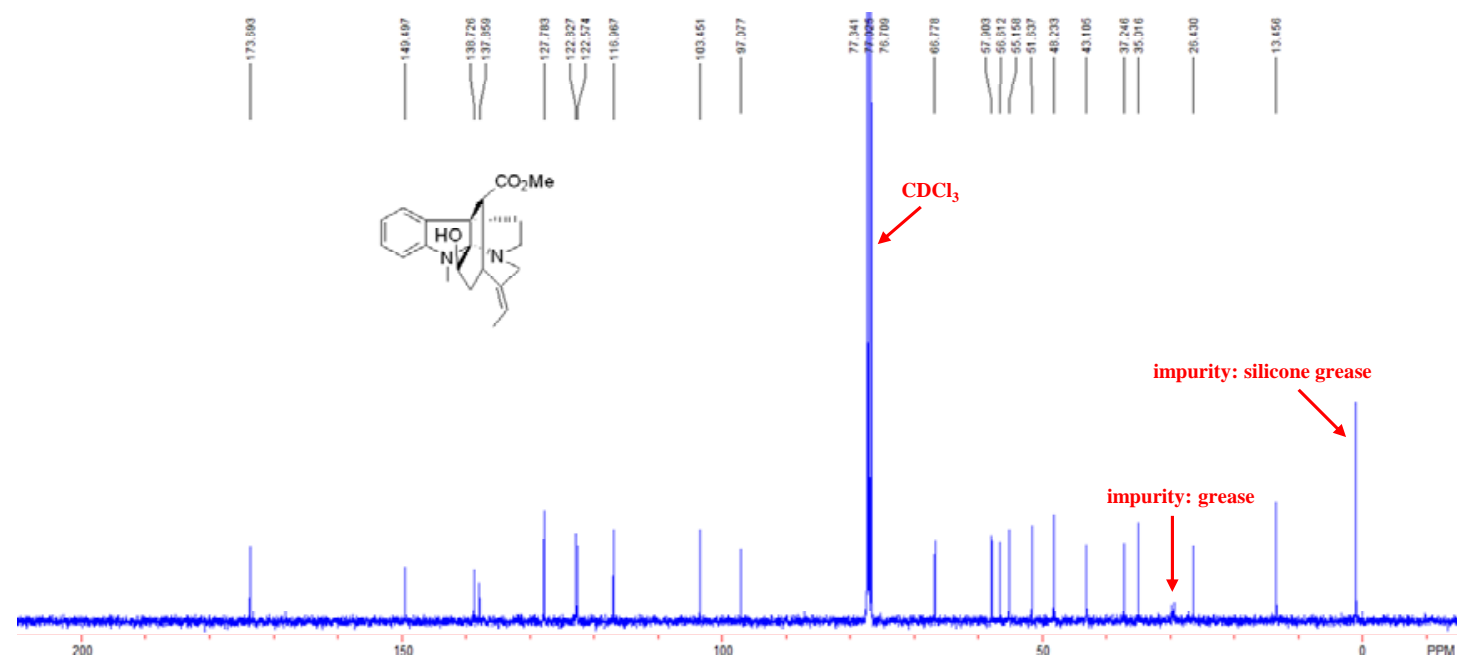

Zu's $\left({ }^{13} \mathrm{C}\right.$ NMR (101 MHz, $\left.\mathrm{CDCl}_{3}\right)$, ( \pm )-deformylcorymine) (Org. Lett. 2017, 19, 5430)

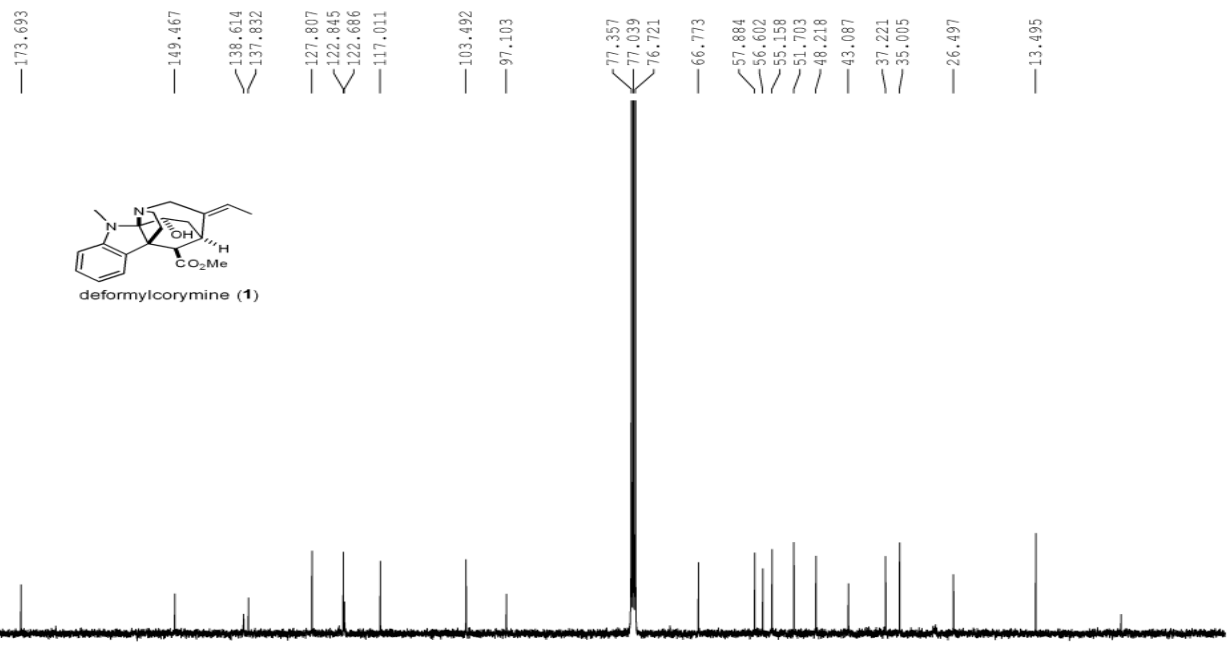

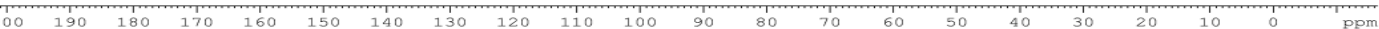

The ${ }^{13} \mathrm{C}$ NMR spectrum of natural deformylcorymine is not available. The reported data are copied as follows:

\begin{tabular}{crcr}
\hline Carbon & $3^{\text {b) }}$ & Carbon & \multicolumn{1}{c}{$\mathbf{3}^{\text {b) }}$} \\
\hline 2 & 97.04 & 14 & 37.22 \\
3 & 66.76 & 15 & 34.99 \\
5 & 55.13 & 16 & 48.21 \\
6 & 43.08 & 17 & - \\
7 & 56.59 & 18 & 13.44 \\
8 & 137.83 & 19 & 122.80 \\
9 & 122.53 & 20 & 138.70 \\
10 & 116.93 & 21 & 57.88 \\
11 & 127.75 & $\mathrm{CO}$ & 173.66 \\
12 & 103.42 & $\mathrm{OMc}$ & 51.62 \\
13 & 149.45 & $\mathrm{NMe}$ & 26.40 \\
\hline b) In $\mathrm{CDCl}_{3}$. & & &
\end{tabular}




\section{(c) 10-Demethoxyvincorine, comparison of spectra}

Our's ( ${ }^{1} \mathrm{H}$ NMR (400 MHz, $\left.\mathrm{CDCl}_{3}\right),(-)$-10-demethoxyvincorine)

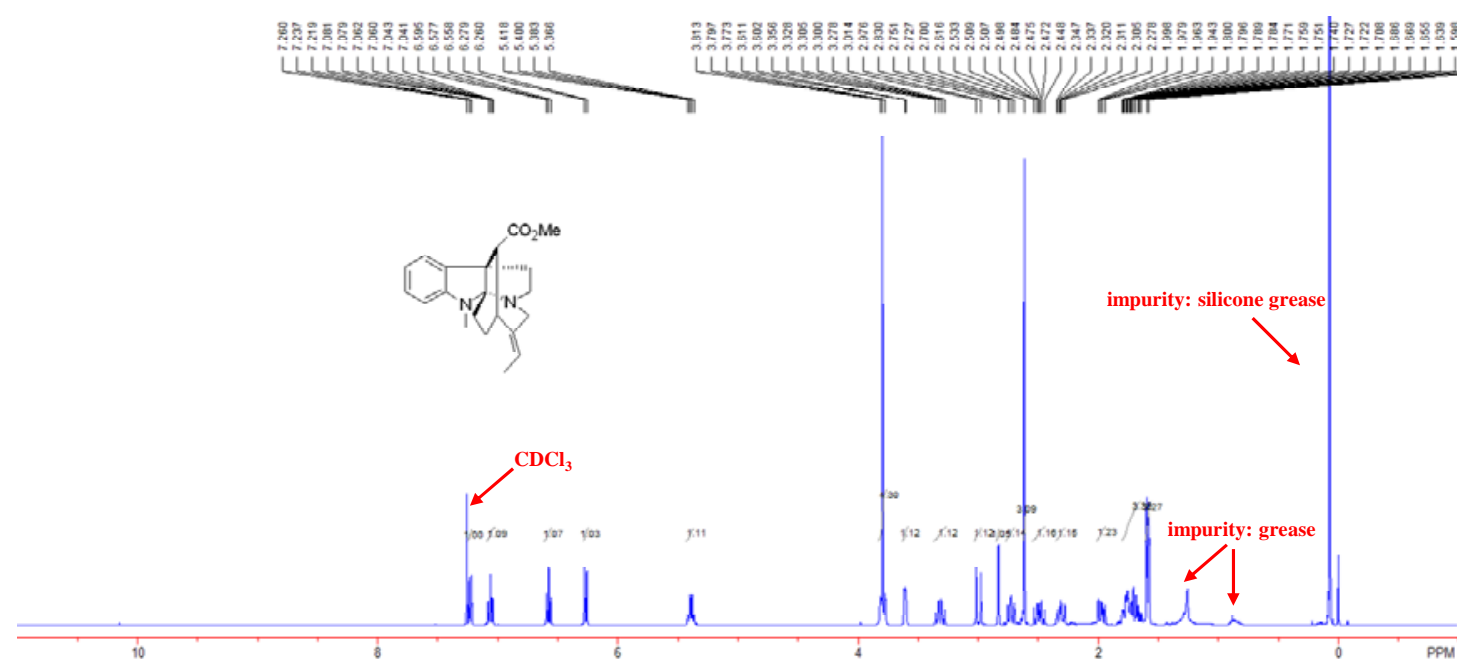

Garg's ( ${ }^{1} \mathrm{H}$ NMR (500 MHz, $\left.\mathrm{CDCl}_{3}\right)$, (-)-10-demethoxyvincorine) (J. Am. Chem. Soc. 2018, 140, 6483)

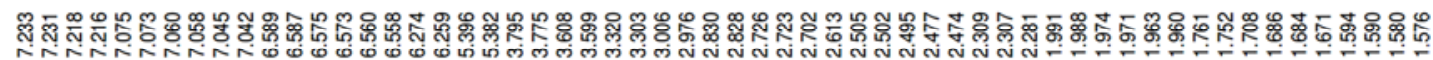
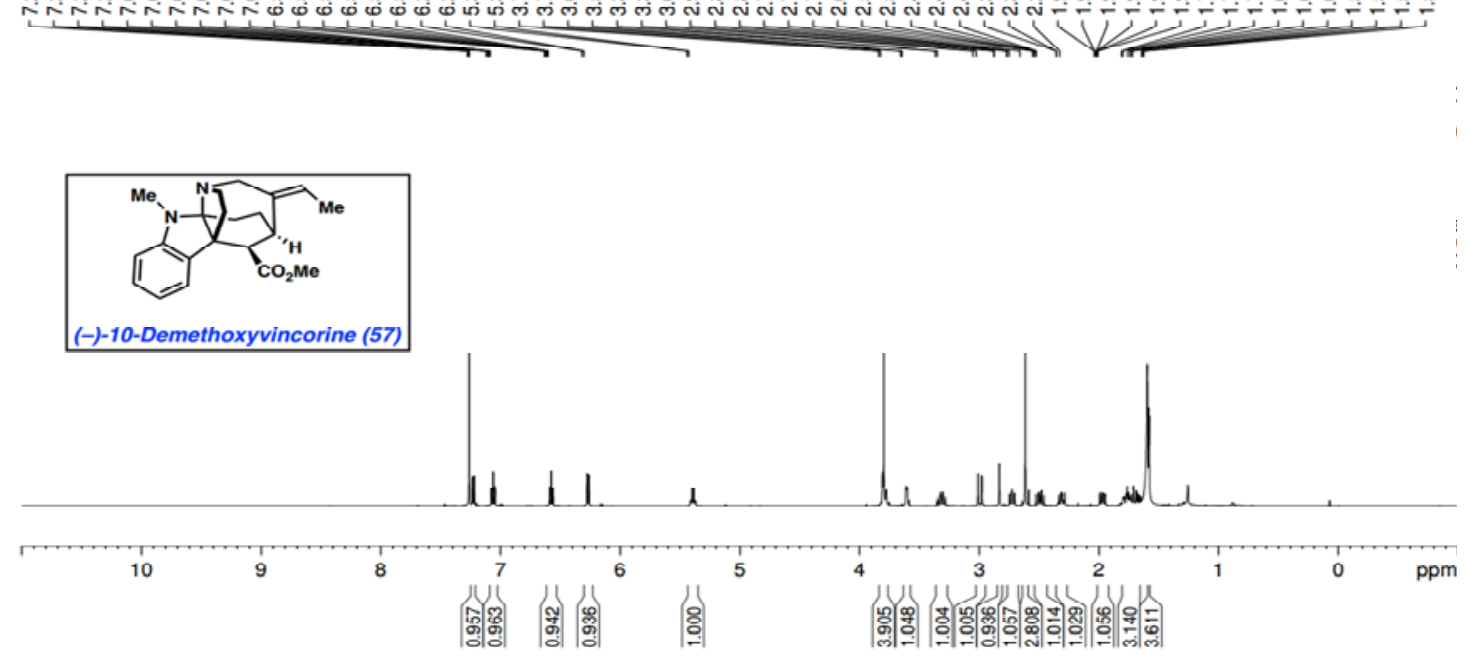

The ${ }^{1} \mathrm{H}$ NMR spectrum of natural deformylcorymine is not available. The reported data are copied as follows:

\begin{tabular}{llll}
\hline $\mathrm{H}$ & \multicolumn{1}{c}{$\mathbf{1 9}$} & $\mathrm{H}$ & \multicolumn{1}{c}{$\mathbf{1 9}$} \\
\hline 3 & $1.68 \mathrm{~m}(\mathrm{~b})$ & $14 \mathrm{~b}$ & $1.76 \mathrm{~m}$ \\
& $2.32 \mathrm{~m}(\mathrm{a})$ & $14 \mathrm{a}$ & $1.76 \mathrm{~m}$ \\
5a & $2.73 \mathrm{br} \mathrm{t}(11)$ & 15 & $3.61 \mathrm{~m}$ \\
$5 \mathrm{~b}$ & $3.33 \mathrm{td}(11,9)$ & 16 & $2.83 \mathrm{br} \mathrm{s}$ \\
6b & $2.50 \mathrm{ddd}(14,11,9)$ & 18 & $1.59 \mathrm{dd}(7,2)$ \\
$6 \mathrm{a}$ & $1.97 \mathrm{ddd}(14,9,1)$ & 19 & $5.40 \mathrm{q}(7)$ \\
9 & $7.22 \mathrm{dd}(7.5,1)$ & $21 \mathrm{a} 3.00 \mathrm{br} \mathrm{d}(15)$ \\
10 & $6.58 \mathrm{td}(7.5,1)$ & $21 \mathrm{~b} 3.81 \mathrm{~m}$ \\
11 & $7.06 \mathrm{td}(7.5,1)$ & $\mathrm{N}_{1}-\mathrm{Me} 2.63 \mathrm{~s}$ \\
12 & $6.27 \mathrm{brd}(7.5)$ & $\mathrm{CO}_{2} \mathrm{Me} 3.79 \mathrm{~s}$
\end{tabular}

Measured in $\mathrm{CDCl}_{3}$. 
Our's $\left({ }^{13} \mathrm{C}\right.$ NMR (101 MHz, $\left.\mathrm{CDCl}_{3}\right),(-)$-10-demethoxyvincorine)

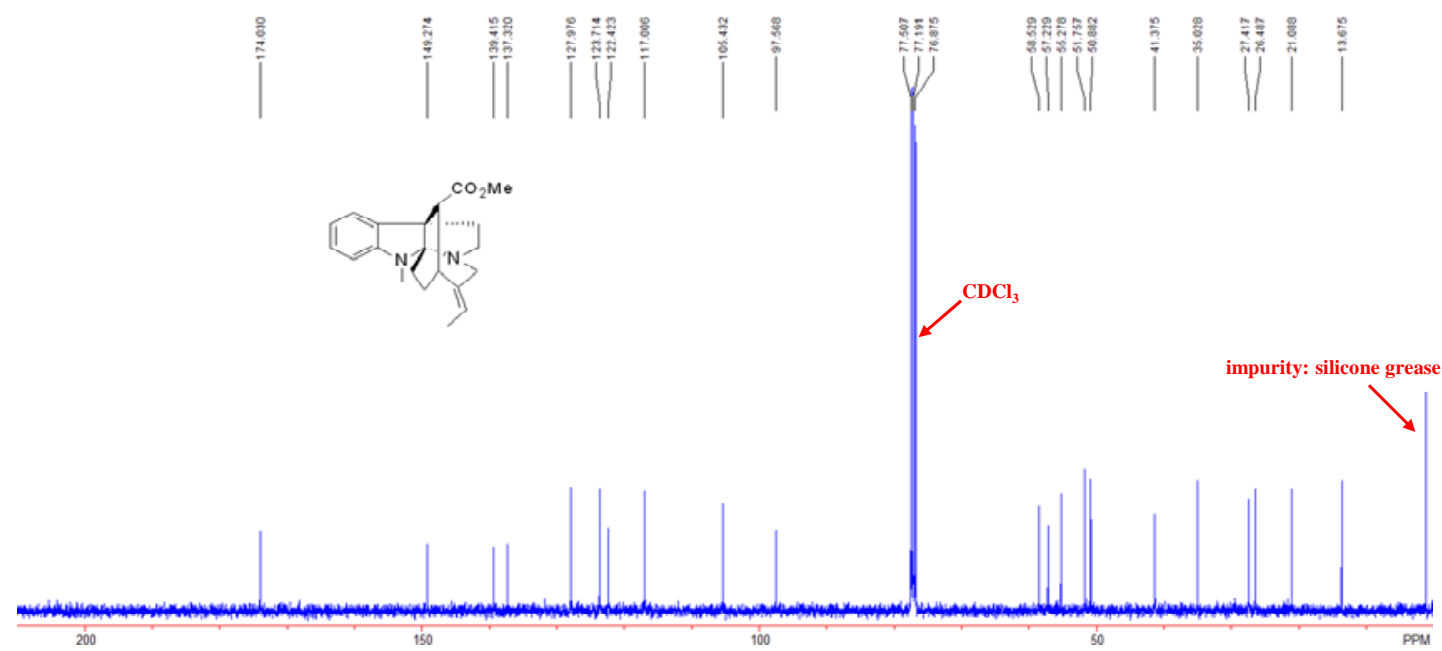

Garg's $\left({ }^{13} \mathrm{C}\right.$ NMR $\left(125 \mathrm{MHz}, \mathrm{CDCl}_{3}\right),(-)-10$-demethoxyvincorine) (J. Am. Chem. Soc. 2018, 140, 6483)
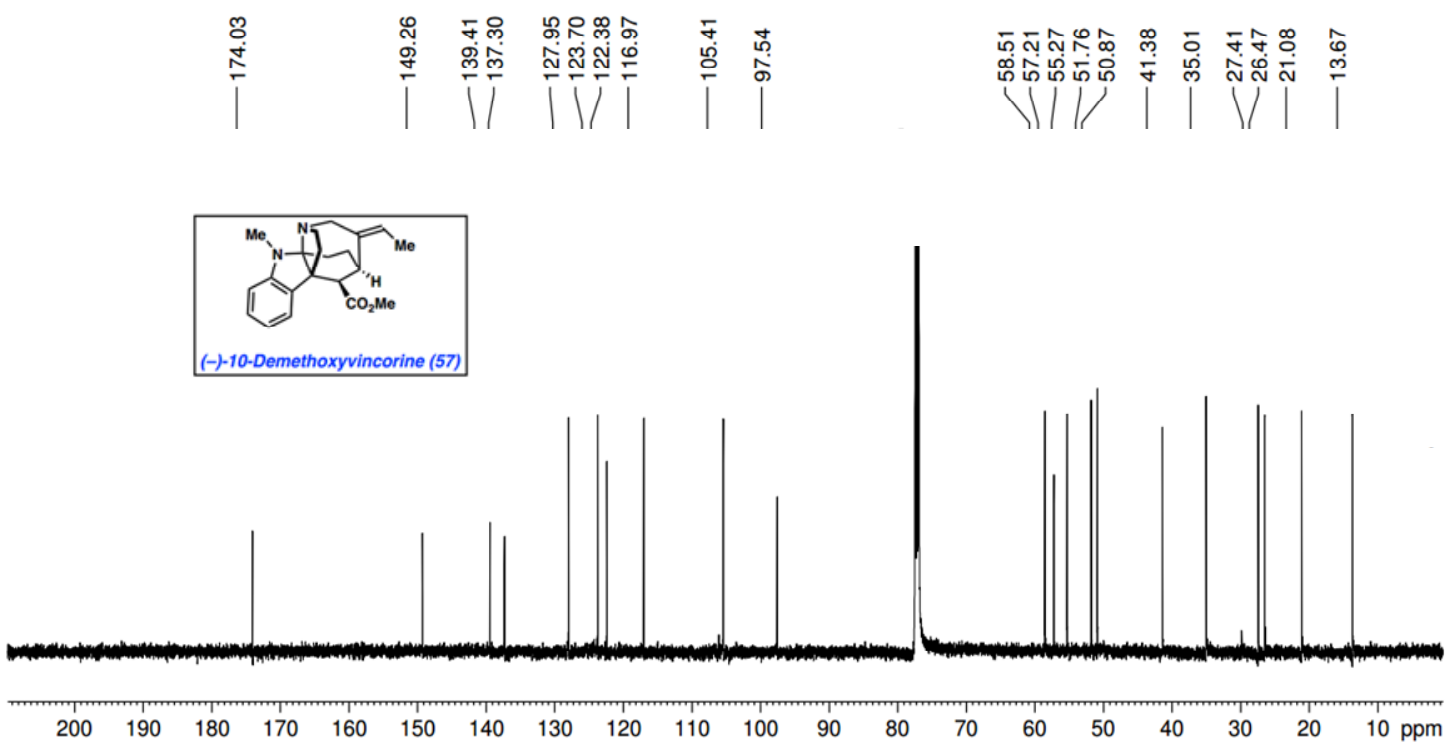

The ${ }^{13} \mathrm{C}$ NMR spectrum of natural 10-demethoxyvincorine is not available. The reported data are copied as follows:

\begin{tabular}{|c|c|c|c|}
\hline$C$ & 19 & $C$ & 19 \\
\hline 2 & 97.4 & 14 & 26.2 \\
\hline 3 & 20.8 & 15 & 34.8 \\
\hline 5 & 55.0 & 16 & 50.6 \\
\hline 6 & 41.0 & 17 & - \\
\hline 7 & 57.0 & 18 & 13.5 \\
\hline 8 & 137.0 & 19 & 122.4 \\
\hline 9 & 123.5 & 20 & 138.9 \\
\hline 10 & 116.9 & 21 & 58.3 \\
\hline 11 & $127.8 \mathrm{~N}$ & $\mathrm{~N}_{1}-M e$ & 27.3 \\
\hline 12 & 105.3 & OMe & 51.5 \\
\hline 13 & 149.0 & $\mathrm{CO}$ & 173.8 \\
\hline
\end{tabular}


(d) 3-epi-Dihydrocorymine 17-acetate, comparison of spectra

Our's ( ${ }^{1} \mathrm{H}$ NMR (400 MHz, $\left.\mathrm{CDCl}_{3}\right)$, (-)-3-epi-dihydrocorymine 17-acetate)

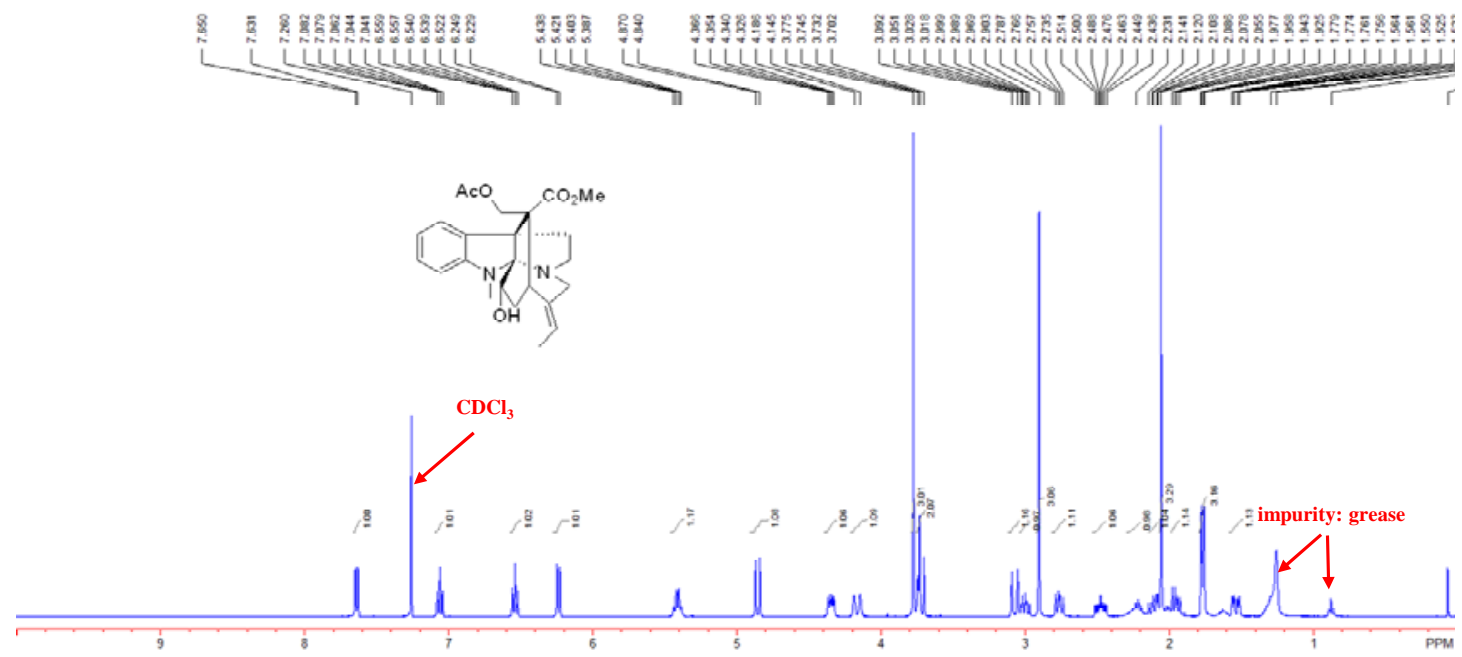

The ${ }^{1} \mathrm{H}$ NMR spectrum of natural 3-epi-dihydrocorymine 17 -acetate is not available.

The reported data are copied as follows:

${ }^{1} \mathrm{H}$ NMR $\left(\mathrm{CDCl}_{3}-\mathrm{CD}_{3} \mathrm{OD}\right) \delta 7.60(t, 7 \mathrm{~Hz}), 7.05(d$, $7 \mathrm{~Hz}), 6.55(t, 7 \mathrm{~Hz}), 6.25(d, 7 \mathrm{~Hz}), 5.40(q, 7 \mathrm{~Hz}), 4.85(d$, $12 \mathrm{~Hz}), 3.80(s, 3 \mathrm{H}), 2.90(s, 3 \mathrm{H}), 2.03(s, 3 \mathrm{H}), 1.80(d, 7 \mathrm{~Hz}$, $3 \mathrm{H})$.

The peak at $7.60 \mathrm{ppm}$ should be doublet, and the peak at $7.05 \mathrm{ppm}$ should be triplet. We think the authors reversed the order of these two peaks. We measured the ${ }^{1} \mathrm{H}$ NMR of 3-epi-dihydrocorymine 17 -acetate in different proportions of $\mathrm{CDCl}_{3}$ and $\mathrm{CD}_{3} \mathrm{OD}$. However, the order of these two peaks didn't change at all. The authentic 3-epi-dihydrocorymine 17-acetate can be obtained by acetylation of 3-epi-dihydrocorymine according to Olivier's condition, whose ${ }^{1} \mathrm{H}$ NMR matched well with the 3-epi-dihydrocorymine 17-acetate synthesized by us. 
(e) 2(S)-Cathafoline, comparison of spectra

Our's ( ${ }^{1} \mathrm{H}$ NMR (400 MHz, $\left.\mathrm{CDCl}_{3}\right),(-)-2(S)$-cathafoline)

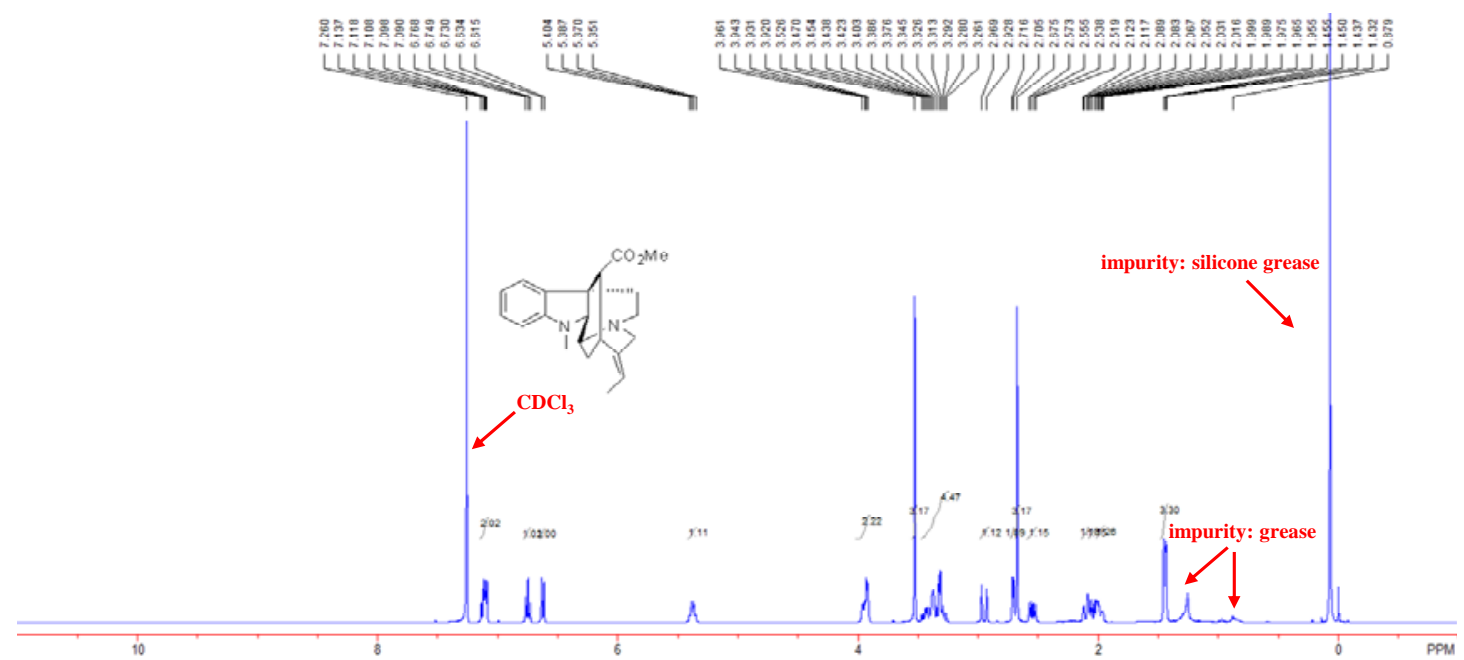

Garg's ( ${ }^{1} \mathrm{H}$ NMR (500 MHz, $\left.\mathrm{CDCl}_{3}\right),(-)-2(S)$-cathafoline) (J. Am. Chem. Soc. 2016, 138, 1162)

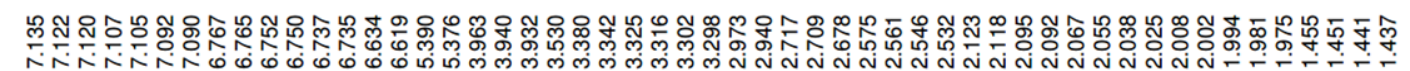

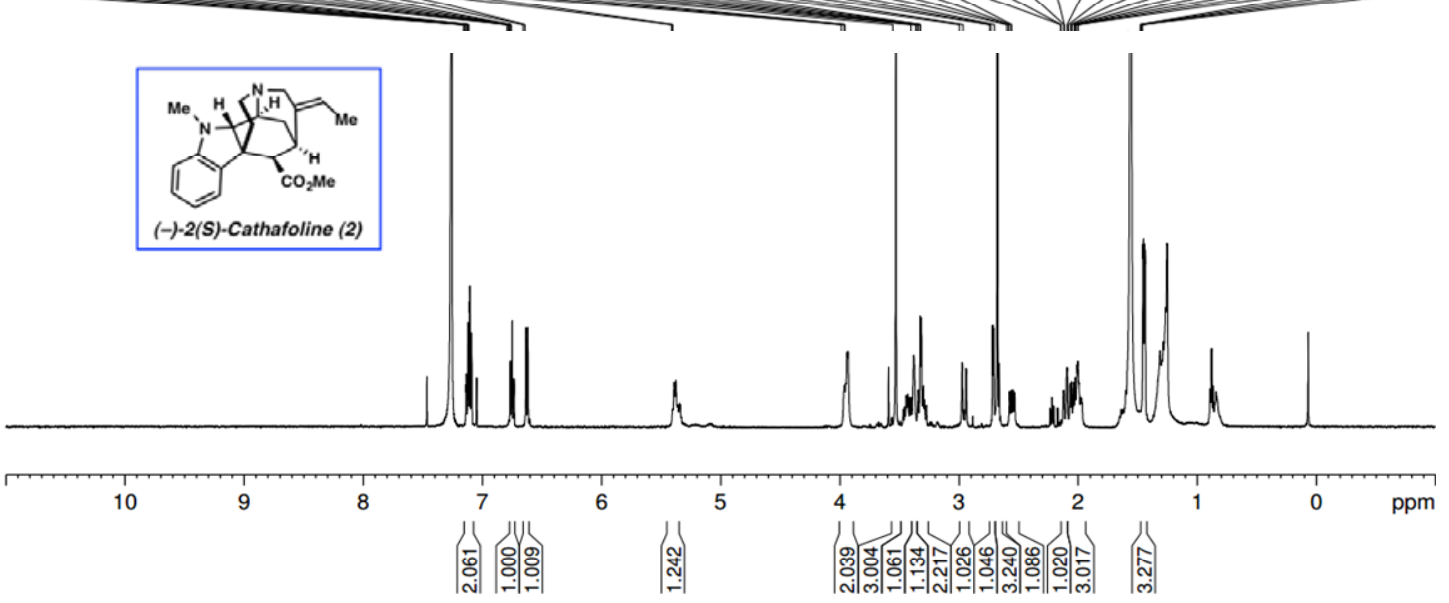

The ${ }^{1} \mathrm{H}$ NMR spectrum of natural 2(S)-cathafoline is not available. The reported data are copied as follows: 


\begin{tabular}{|c|c|c|}
\hline $\mathrm{H}$ & 16 & H 16 \\
\hline 2 & $3.25 \mathrm{~d}(4)$ & 14b $1.99 \mathrm{dt}(15,4)$ (a) \\
\hline 3 & $3.98 \mathrm{~m}$ & $14 \mathrm{a} \cdot 2.13 \mathrm{dd}(15,3)$ (b) \\
\hline $5 a$ & $2.58 \mathrm{dd}(14,7)$ & $153.39 \mathrm{~m}$ \\
\hline $5 b$ & $3.45 \operatorname{td}(14.6)$ & $162.72 \mathrm{~d}(4)$ \\
\hline $6 b$ & $2.07 \mathrm{dd}(14,6)$ & $181.46 \mathrm{dd}(7,2)$ \\
\hline $6 a$ & $3.35 \mathrm{~m}$ & $195.41 \mathrm{q}(7)$ \\
\hline 9 & $6.98 \mathrm{~d}(7)$ & 21a 2.98 d (16) \\
\hline 10 & $6.64 \mathrm{t}(7)$ & 21b 3.95 d (16) \\
\hline 11 & $7.01 \mathrm{t}(7)$ & $\mathrm{N}_{1}-\mathrm{Me} 2.68 \mathrm{~s}$ \\
\hline 12 & $6.51 \mathrm{~d}(7)$ & $\mathrm{CO}_{2} \mathrm{Me} 3.53 \mathrm{~s}$ \\
\hline
\end{tabular}

Our's $\left({ }^{13} \mathrm{C}\right.$ NMR (101 MHz, $\left.\mathrm{CDCl}_{3}\right),(-)-2(\mathrm{~S})$-cathafoline)

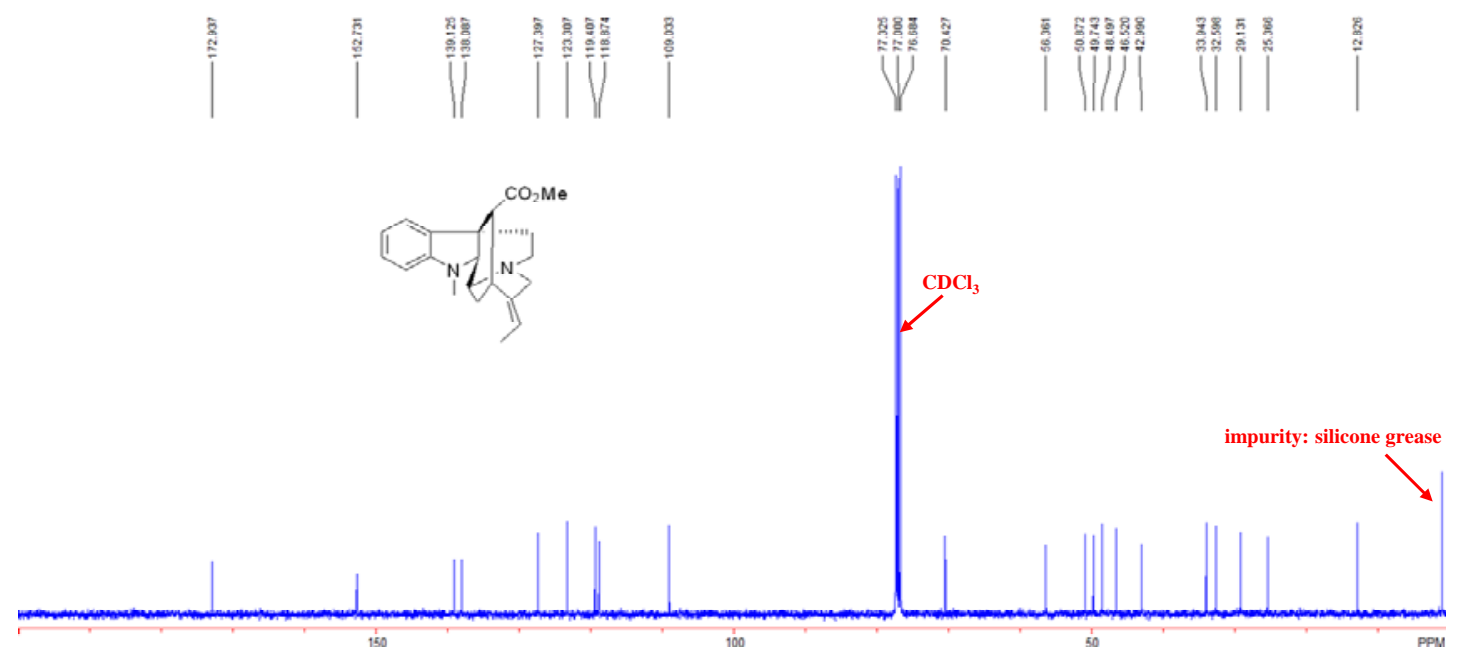

The ${ }^{13} \mathrm{C}$ NMR of Garg's synthetic (-)-2(S)-cathafoline was measured in $\mathrm{C}_{6} \mathrm{D}_{6}$.

The ${ }^{13} \mathrm{C}$ NMR spectrum of natural $2(S)$-cathafoline is not available. The reported data are copied as follows:

\begin{tabular}{llll}
\hline C & 16 & C & 16 \\
\hline 2 & 70.1 & 14 & 25.2 \\
3 & 48.7 & 15 & 32.5 \\
5 & 46.5 & 16 & 49.6 \\
6 & 28.7 & 17 & - \\
7 & 42.9 & 18 & 12.9 \\
8 & 137.8 & 19 & 119.5 \\
9 & 123.3 & 20 & 138.1 \\
10 & 119.5 & 21 & 56.2 \\
11 & 127.5 & $\mathrm{~N}_{1}-M e$ & 34.0 \\
12 & 109.1 & OMe & 51.0 \\
13 & 152.6 & CO & 172.8 Measured in $\mathrm{CDCl}_{3}$.
\end{tabular}




\section{HPLC Traces for Measuring Enantiomeric Excesses}

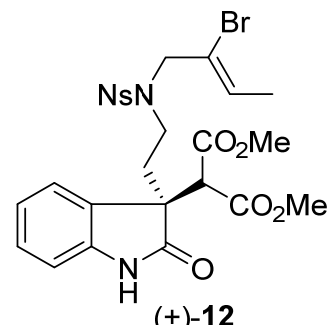

Racemic and enantioenriched 12 were analyzed by HPLC (CHIRALPAK ID column, iPrOH:hexane $36: 64,0.70 \mathrm{~mL} / \mathrm{min}$ ) to determine the enantiomeric excess. For 12, ee $=99 \%$

$\mathrm{mAU}$

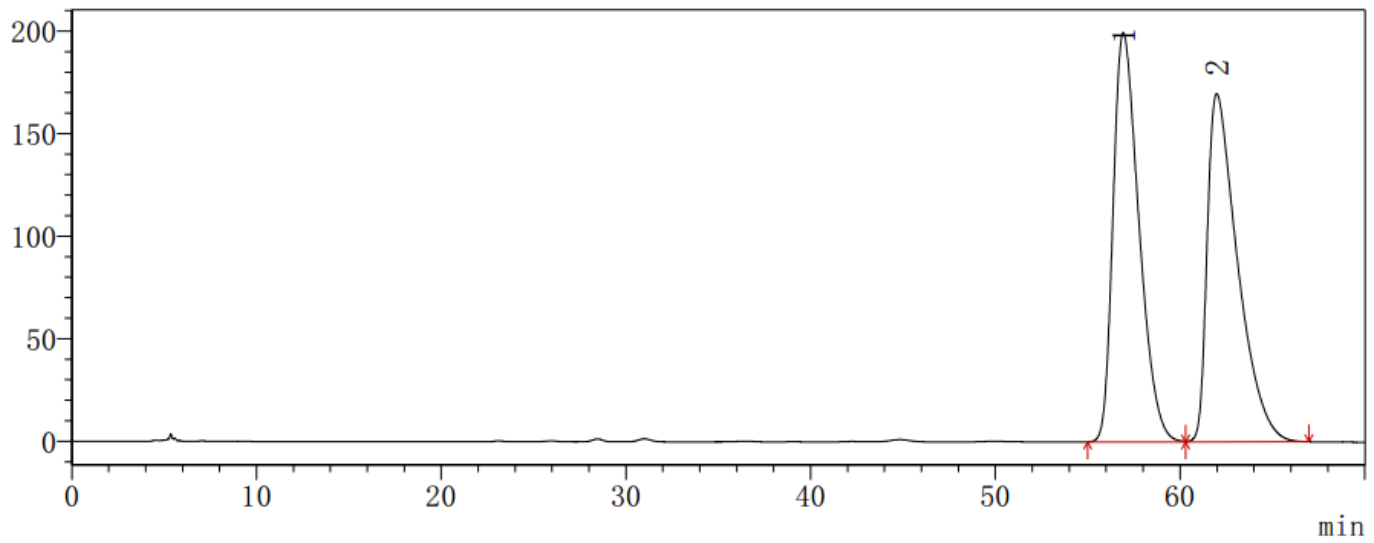

PDA Ch1 254nm

\begin{tabular}{|r|r|c|r|}
\hline Number & Ret. Time & Area & \multicolumn{1}{c|}{ Area\% } \\
\hline 1 & 56.926 & 19427496 & 50.06 \\
\hline 2 & 61.985 & 19384546 & 49.94 \\
\hline
\end{tabular}

$\mathrm{mAU}$

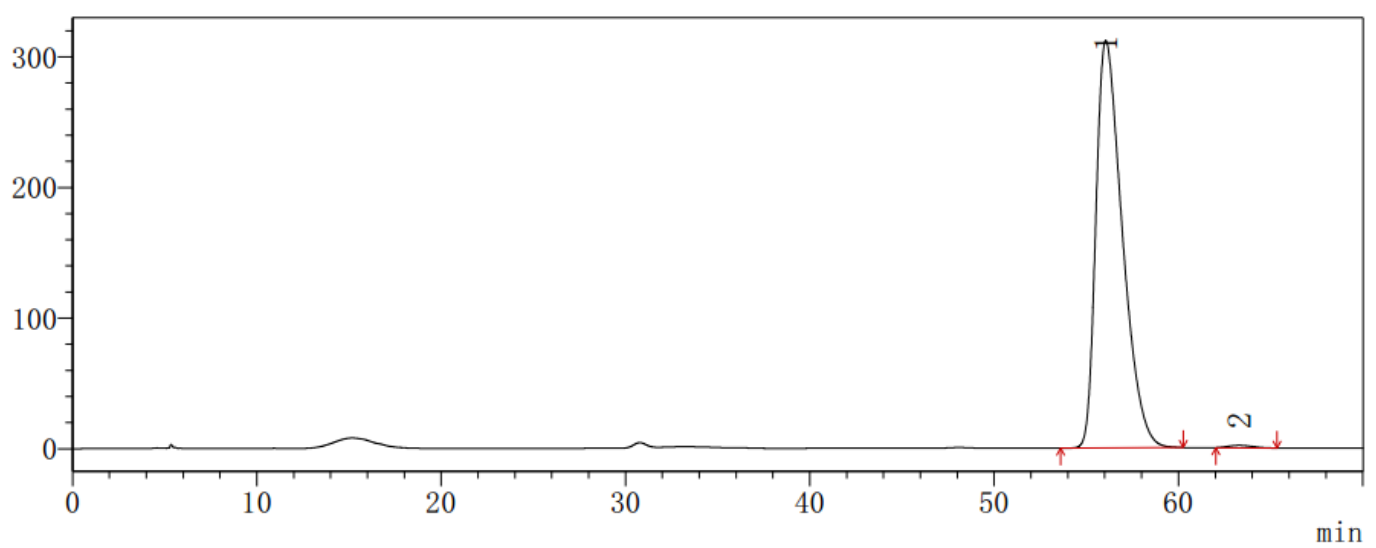

PDA Ch1 254nm

\begin{tabular}{|r|r|c|r|}
\hline Number & Ret. Time & Area & \multicolumn{1}{c|}{ Area\% } \\
\hline 1 & 56.056 & 31024433 & 99.45 \\
\hline 2 & 63.282 & 170094 & 0.55 \\
\hline
\end{tabular}

